\title{
Sensitivity-Analysis Techniques: Self-Teaching Curriculum
}

NUREG/CR- -2350

DE82 017558

Manuscript Completed: January 1982

Date Published: June 1982

Prepared by

R. L. Iman, W. J. Conover*

Sandia National Laboratories

Albuquerque, NM 87185

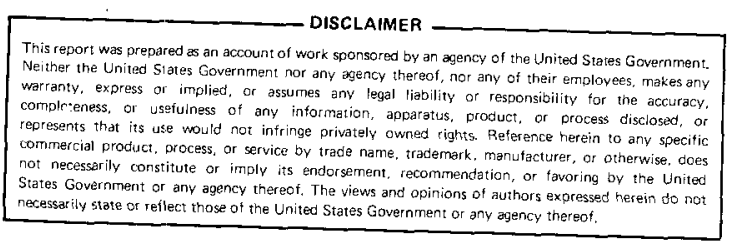

*Texas Tech University

Prepared for

Division of Waste Management

Office of Nuclear Material Safety and Safeguards

U.S. Nuclear Regulatory Commission

Washington, D.C. 20555

NRC FIN A1158

This document is

PUBLICLY RELEASABLE

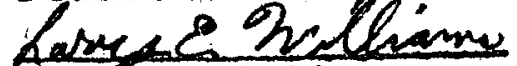

Authofizing Official

Date: $03 / 10 / 2006$

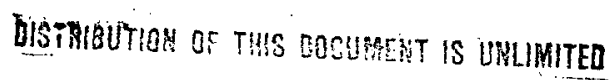

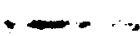




\section{DISCLAIMER}

This report was prepared as an account of work sponsored by an agency of the United States Government. Neither the United States Government nor any agency Thereof, nor any of their employees, makes any warranty, express or implied, or assumes any legal liability or responsibility for the accuracy, completeness, or usefulness of any information, apparatus, product, or process disclosed, or represents that its use would not infringe privately owned rights. Reference herein to any specific commercial product, process, or service by trade name, trademark, manufacturer, or otherwise does not necessarily constitute or imply its endorsement, recommendation, or favoring by the United States Government or any agency thereof. The views and opinions of authors expressed herein do not necessarily state or reflect those of the United States Government or any agency thereof. 


\section{DISCLAIMER}

Portions of this document may be illegible in electronic image products. Images are produced from the best available original document. 
This self teaching curriculum on sensitivity analysis techniques consists of three parts:

1) Use of the Latin Hypercube Sampling Program [Iman, Davenport and Ziegler, Latin Hypercube Sampling (Program User's Guide), SAND79-1473, January 1980].

2) Use of the Stepwise Regression Program [Iman, et al., Stepwise Regression with PRESS and Rank Regression (Program User's Guide) SAND79-1472, January 1980].

3) Application of the procedures to sensitivity and uncertainty analyses of the groundwater transport model NWFT/DVM [Campbell, Iman and Reeves, Risk Methodology for Geologic Disposal of Radioactive Waste - Transport Model Sensitivity Analysis;

SAND80-0644, NUREG/CR-1377, June 1980: Campbel1, Longsine, and Reeves, The Distributed Velocity Method of Solving the Convective-Dispersion Equation, SAND80-0717, NUREG/CR-1376, July 1980].

This curriculum is one in a series developed by Sandia National Laboratories for transfer of the capability to use the technology developed under the NRC funded High Level Waste Methodology Development Program (NRC FIN. No. A-1192). The technology transfer process is carried out under NRC Fin No. A-1158. 


\section{TABLE OF CONTENTS}

Page

PART ONE TUTORIAL ON THE LATIN HYPERCUBE SAMPLING PROGRAM 1

The Purpose of the Course

The Importance of Good Sampling Techniques

The Nuclear Waste Repository Model

Several Types of Input Distributions

The Importance of Accurate Input Distributions

The Simplified Black Box Model

Objectives of a Simulation Study

To assess the probability of the

output exceeding specified limits

To determine the sensitivity of the

output to the various input variables

On the Latin Hypercube Sampling Program

Options for Input Distributions

The Distribtution Function vs. the Density Function

Drawing a Random Sample (I1lustration)

1. Draw $N=2$ random uniform values

2. Convert these to numbers between 0 and 1

3. Use Figure 8 to find $F^{-1}$ for these numbers

Drawing a Stratified Sample (Illustration)

1. Draw $N=2$ random uniform values

2. Convert the first number to a number between 0 and .5 and the second to a number between .5 and 1.0

3. Use Figure 9 to find $F^{-1}$ for these numbers 12

More Exact Normal Values from Table 2

Exercise 1: Drawing a Random Sample of Size

$$
N=4 \text { from } F(x)
$$

Exercise 2: Drawing a Latin Hypercube Sample of Size $N=4$ from $F(x)$ 
Exercise 3: More Accurate Figures for Exercise 1

Page

Exercise 4: More Accurate Figures for Exercise 2

Answer to Question 1:

Obtaining a Multivariate Random Sample

Obtaining a Latin Hypercube Sample

Comparing Latin Hypercube With Random Sampling

Obtaining a Multivariate Random Sample (Illustration)

Exercise 5: Multivariate Random Sample of Size 10

The Accuracy of the Output From a Random Sample

obtaining a Random Permutation

Obtaining a Latin Hypercube Sample (I1lustration)

Step 1. Obtain uniform random numbers in each of $\mathrm{N}$ Strata

Step 2: Arrange the values of $F(x)$ in a random order

Step 3: Convert the values of $F(x)$ to a Stratified sample from $F(x)$

Step 4: Combine the individual stratified samples into a Latin Hypercube Sample

Step 5: Obtain the output from the black box model using the Latin Hypercube Sample 36

Step 6: Plot an empirical distribution function 36

Exercise 6: Obtaining a Latin Hypercube Sample of Size 10

Accuracy Obtained from Using a Latin Hypercube Sample 43

A Comparison of Latin Hypercube with Random Sampling 43

The Replicated Latin Hypercube Sample 43

Estimating Other Population Parameters 48

Changes in the Input Distributions 48

Illustrating the Effect of a Change in Input

Distributions

The Actual Correlation on the Input values 
The Rank Correlation on the Input Values

Page

Some Undesirable Effects of Spurious Correlation

Reducing the Spurious Correlation

An Illustration of Reducing the Correlation

Simulating Correlated Input Variables

Illustration of Correlating Input Variables

A New Output Distribution Function

How Many Runs Are Needed

The Purpose of the Course

The Need for Regression Methods

Simple Linear Regression

The Method of Least Squares

The Least Squares Equations

Example

Rank Regression

Converting Predicted Ranks to Predicted Values

The Residuals Sum of Squares (SS)

Exercise 1: Find Predicted Value $Y=4.85$

from Rank Regression Example

The Flexibility of Rank Regression for Fitting Monotonic Data

An Example. With Real Data

Comparing Rank Regression With Ordinary Regression

Multiple Regression

An Example of Multiple Regression

Ordinary Multiple Regression Illustrated

Exercise 2: Verification of Predicted Values in Figure 13

Rank Multiple Regression Illustrated 
TABLE OF CONTENTS ( cont'd)

Page

Exercise 3: Obtaining Predicted Rank by Substituting Data in Figure 14 into' Equation 9

Exercise 4: Using Predicted Ranks to Obtain Predicted Values for $Y$

Comparing Ordinary Regression and Rank Regression 84

Sensitivity Analys is $\quad 84$

On Deciding What Variables to Include in the Model 86

Simple Correlation $\quad 86$

Rank Correlation $\quad 86$

Partial Correlation $\quad 87$

An Equation for Computing Partial Correlation 87

$\begin{array}{ll}\text { Partial Rank Correlation } & \mathbf{8 8}\end{array}$

Partial Correlation Given Several Variables 88

Three Multiple Regression Procedures 89

1. The forward procedure 89

2. The backward procedure 89

3. The stepwise procedure 89

The Variables Being Considered 89

The Forward Procedure $\quad 90$

A Test of Significance $\quad 90$

Using Partial Correlation in Forward Regression 90

Exercise 5: Finding a Partial Correlation Coefficient 90

Adding Another Variable to the Model 91

The Forward Regression Model 91

Obtaining Predicted Values in Multiple Regression 92

The Forward Rank Regression Procedure 92

Using Rank Regression to Predict Values of $Y \quad 94$

Exercise 6: Finding a Value for $r_{y} \quad 95$

Exercise 7: Obtaining a Predicted Value for Y Given $r_{y} 95$

Comparing Rank Regression with Ordinary Regression 95

Backward Regression $\quad 95$ 
A Useful Procedure for Finding Partial

Correlation Coefficients

An Illustration of the Procedure 96

Measuring the Importance of Variables 97

The Results Using Backward Regression : 97

Backward Rank Regression 98

The Model from Backward Rank Regression 98

A Comparison of the Several Models 98

Stepwise Regression $\quad 99$

Di scussion $\quad 99$

$\begin{array}{ll}\text { References } & 101\end{array}$

PART THREE APPLICATION OF THESE PROCEDURES TO SENSITIVITY AND UNCERTAINTY ANALYSES OF THE GROUND WATER TRANSPORT MODEL NWFT/DVM

Example of Setting Up and Executing the Latin Hypercube Sampling Program Along with Output from a Transport Model

Parameter Cards Used to Generate Latin Hypercube 104 Subroutine USRDIST 105

LHS NWFT DVM for NRC Short Course: 106

Total Integrated Di scharge to 104 Years 112

An Example of Sensitivity Analysis Results Based on the Partial Rank Correlation Coefficient

Partial Rank Correlation Figures :

Stepwise Regression Analysis for the Previous Example of this Section Using all 105 Observations

Transformations to Create New Variables (such as Retardation Factors)

Stepwise Regression Program 136

Summary of Stepwise Regression on Raw Data 145

Summary of Stepwise Regression on Ranks 146 
PART ONE:

Figure No.

Page No.

Some Input Variables for a Hypothetical Nuclear Waste Repository

Input Variables and Their Distributions for a Hypothetical Black Box Model

A Normal Density Function $f(x)$ and its Corresponding Distribution Function $F(x)$

A Uniform Density Function $f(x)$ and its Corresponding Distribution Function $F(x)$

A Lognormal Distribution Function $f(x)$ and its Corresponding Distribution Function $F(x)$

A Loguniform Density Function $f(x)$ and its Corresponding Distribution Function $F(x)$

A Distribution Function Estimated from a Large Number of Sample Observations

A Hypothetical Distribution Function $F(x)$ for Use in Drawing a Random Sample of Size $N=2$

A Hypothetical Distribution Function $F(x)$ for Use in Drawing a Stratified Sample of Size $N=2$

10 Worksheet for Drawing a Random Sample of Size $N=4$ from $F(x)$

11 A Hypothetical Distribution Function $F(x)$ for Use in Drawing a Random Sample of Size $N=4$

Worksheet for Drawing a Latin Hypercube Sample of Size $N=4$ from $F(x)$

A Hypothetical Distribution Function $F(x)$ for Use in Drawing a Latin Hypercube Sample of Size $N=4$ 


\section{LIST OF FIGURES (Continued)}

Page No.

14 Worksheet for Drawing a Random Sample of

Size 10 for $X_{1}$, where $x_{1}$ is Normal, $\mu=1, \sigma=1$

Worksheet for Drawing A Random Sample of

Size 10 for $X_{2}$, where $x_{2}$ is Normal, $\mu=2, \sigma=1$

Worksheet for Drawing A Random Sample of

Size 10 for $X_{3}$, where $X_{3}$ is Normal, $\mu=2, \sigma=1$

Worksheet for Drawing A Random Sample of

Size 10 for $X_{4}$, where $X_{4}$ is Normal, $\mu=3, \sigma=1$

The Multivariate Input Vectors Using a Random

Sample, and the Corresponding Output

An Empirical Distribution Function from the Random Sample of Figure 18

Five Empirical Distribution Functions (a)-(e) from Random Samples of Size 10, and an Estimate of the Population Distribution Function

21 A Graph of the Mean of the Five EDFs from Figure 19, and an Estimate of the Population Distribution Function

The Mean and One Standard Deviation Bounds of the Five EDFs from Figure 20

A Stratified Sample of Size 10 for $x_{1}$ from a Normal Population with $\mu=1$ and $\sigma=.1$

A Stratified Sample of Size 10 for $x_{2}$ from a Normal Population with $\mu=2$ and $\sigma=1$

A Stratified Sample of Size 10 for $X_{3}$ from $a$ Normal Population with $\mu=2$ and $\sigma=1$

A Stratified Sample of Size 10 for $x_{4}$ from a Normal Population with $\mu=3$ and $\sigma=1$

A Latin Hypercube Sample of Size 10 and the Associated Output $Y$ 


\section{LIST OF FIGURES (Continued)}

Page No.

An Empirical Distribution Function for the Output in Figure 27

Student Problem: Generate 4 Stratified Samples, the Latin Hypercube Sample and an Empirical Distribution Function

Five EDFs Obtained from Latin Hypercube Samples of Size 10 each, and an Estimate of the Population Distribution Function

The Mean of the Five EDFs from Figure 35, and an Estimate of the Population Distribution Function

The Mean of the Five EDFs from Figure 35, and One Standard Deviation Bounds

The Mean of Five EDFs from Figure 20 and One Standard Error Bounds for a Random Sample of Size $N=50$

The Mean of Five EDFs from Figure 35 and One Standard Error Bounds for a Replicated Latin Hypercube Sample of Total Size $N=50$

The Output Distribution Function When the Inputs are Uniformly Distributed, Contrasted with the Case of Normal Input Variables

The Correlation Matrix for the Latin Hypercube Sample in Figure 27

The Ranks of the Input Variables in the Latin Hypercube Sample of Figure 27

The Rank Correlation Matrix for the Ranks in Figure 42

The Rank Ordering Induced on the Random Sample of Size $N=10$, Whose Output EDF is Given in Figure $20(a)$ 
Page No.

45 The Rank Correlation Coefficients for the Ranks in Figure 44

Changing the Order of the Values of $x_{1}$ to Reduce the Spurious Correlation

A Target Correlation Matrix for Four Input Variables

Rank Orderings to Achieve Correlations Close to Those in Figure 47

Sample Rank Correlation Matrix for Ranks in Figure 48

New Input Values with Rank Correlation Matrix Given by Figure 49
An Example of the Differences in output Distributions Obtained, Assuming Input Variable Independence and Assuming a Correlation Between Input Variables

PART TWO:

1 Worksheet for Finding $\underline{a}$ and $\underline{b}$ Using Least Squares

A Graph of the Data in Figure 1, and the Least Squares Regression Line

The Residuals and Sum of Squares from Figure 2

4 Worksheet for Least Squares on the Ranks

A Graph of the Ranks from Figure 4 and the Least Squares Line on the Ranks

Worksheet for Finding Residuals from Rank Regression

A Graph of the Data in Figure 1, and the Rank Regression Curve 
Page No.

8 Nine Values from the Function $Y=e^{X}$

9 Rank Regression Curve and the Least Squares

Line for 9 Points from the Relationship

$y=e^{x}$

10 Graph of the Ranks of $X$ and $Y$ as Given in Figure 9

11 A Comparison of Least Squares Linear Regression on the Data with Regression on the Ranks, Using Data from Daniel and Wood (1971)

12 Multivariable Data from Brownlee (1965)

13 Predicted Values and Residuals Using Least Squares Regression and Rank Regression, on the Data from Figure 12, Using the Variables $x_{1}, x_{2}$ and $x_{3}$

14 The Ranks of the Data in Figure 13 and the Predicted Ranks from Equation (9)

15 Some Correlation Coefficients from the Data from Figure 12 and the Ranks from Figure 14

Predicted Values and Residuals from the Forward Regression Models on the Data and on the Ranks

17 Predicted Values and Residuals Using Forward Rank Regression and Backward Rank Regression 


\section{LIST OF TABLES}

PART ONE

Table No.

Page No.

1 A Table of Uniform Random Numbers

62

2 The Cumulative Standard Normal Distribution

63 
TUTORIAL ON THE LATIN HYPERCUBE SAMPL ING PROGRAM

\section{The Purpose of the Course}

The purpose of this tutorial is to demonstrate how to draw multivariate random samples, using either Random Sampling or using Latin Hypercube Sampling, where the multivariate random sample may have any specified marginal distributions and any specified correlation matrix. This tutorial shows how to obtain such a sample manually, and how to use the Latin hypercube computer prograin to accomplish the same task. A comparison between Random Sampling and Latin Hypercube Sampling is made to show some of the relative benefits of using a Latin Hypercube Sample.

The Importance of Good Sampling Techniques

Some physical processes are difficult to study directly, for various reasons, and are therefore observed indirectly through the use of mathematical models: The mathematical models are often so complex that they are amenable to solution only through the use of numerical methods on a computer. Even then the solution may involve a considerable amount of computer time, so care is needed in selecting the input variables in such a way that the most important information is obtained concerning the output variable. This suggests the use of efficient statistical methods for the design and analysis of pseudo-data generated through the use of such models. Some of those methods are described in detail in the following sections.

The Nuclear Waste Repository Model

Consider the model for simulating geologic conditions in a repository for nuclear waste. The input variables may be random variables or may be parameters whose values are unknown but may be known to lie in given intervals with specified probabilities. In either case, the input variables are subject to uncertainties that may be described by means of a probability distribution. In Figure 1 a diagram of a hypothetical nuclear waste repository is given, and four input variables are shown along with their hypothesized probability distributions. Each probability distribution is specified by name and by the lower limit a and upper limit $b$. In the normal distribution $a$ and $b$ represent truncation of the usual normal distribution at the .00I and .999 quantiles. The upper limit for the lognormal and loguniform distributions are taken to be the .999 quantiles. The convenience of working with a finite range for each variable considerably outweighs the disadvantage of working with a slightly truncated form of a standard distribution. 
Several Types of Input Distributions

The normal distribution and the uniform distribution are wel 1 known distributions which are symmetric. Typical density functions for these two distributions are given in Figure 1. The lognormal distribution is a unimodal distribution, skewed to the right, which is often used to represent random variables that assume only positive values. The logarithm of a lognormally distributed random variable is a random variable with a normal distribution. A loguniform distribution has many of the same properties as a lognormal distribution, such as being unimodal, skewed to the right, and nonnegative. The shape of the distribution is slightly different, with the right tail of the distribution being considerably heavier than the right tail of a lognormal distribution. If a random variable has a loguniform distribution, its logarithm has a uniform distribution. Instead of these four distributions, any probability distribution may be specified for the input variables. These distributions are simply the ones used most frequently in this type of model.

The Importance of Accurate Input Distributions

In any model such as the waste isolation model the output from the model is the item of interest. There are uncertainties attached to the output because there are uncertainties inherent in the input. Thus the output is expressible only in terms of its probability distribution, or properties such as the mean, standard deviation, median, quartiles or other quantities. An accurate representation of the output requires an accurate representation of the input. Therefore good answers to questions concerning the output require accurate representations of the input distributions. Since the quantities represented by the input variables may possess some correlation in nature, that same correlation should be reflected in the selection of input variables for the model. The requirements imposed thus far, i.e., specified distributions on each variable and specified correlations between variables, require some nonstandard statistical methodology. The methods presented in this tutorial have been developed specifically for models such as this one, so they will probably not be familiar to the reader.

The Simplified Black Box Model

In order to present the statistical methodology in a clear, uncluttered manner, details which are not relevant to the statistical methodology are suppressed in this tutorial. One such detail is the model itself. Although the model is the most important link in the study of a physical system, the proper development and verification of the model is the responsibility of geologists, physicists, engineers and other experts. From the statistician's viewpoint the model is viewed as a "black box," with many input variables and one or more output variables. Figure 2 shows a diagram of the model from a statistician's viewpoint, 


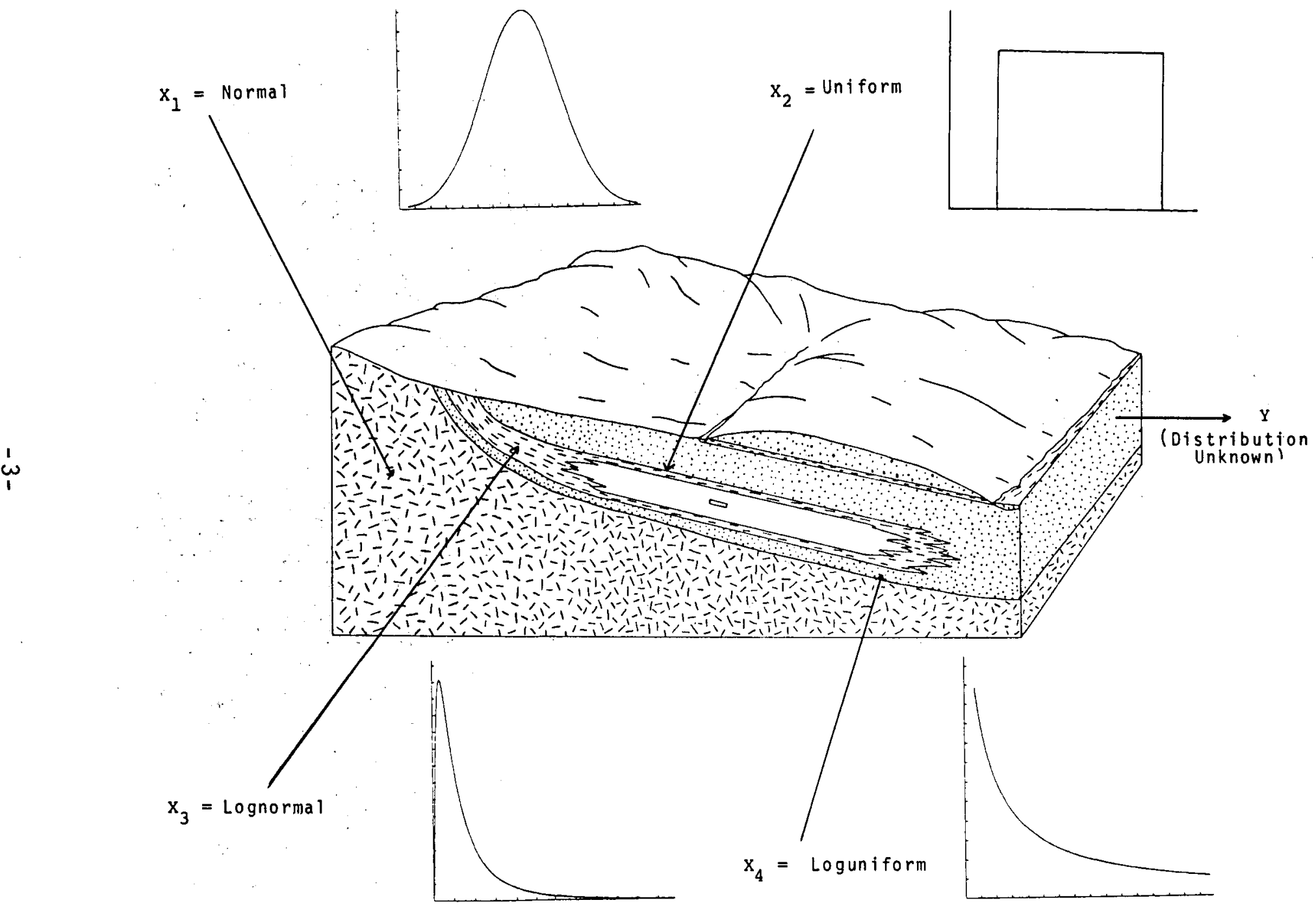

Figure 1. Some Input Variables for a Hypothetical Nuclear Waste Repository. 


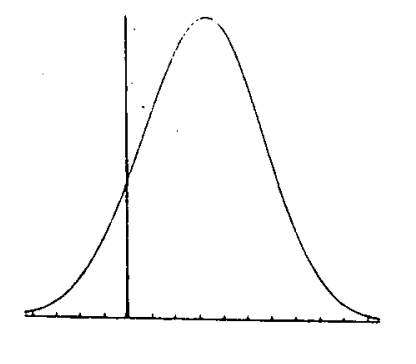

$\mathrm{x}_{1}=$ Normal

$(a=2.09, b=4.09)$

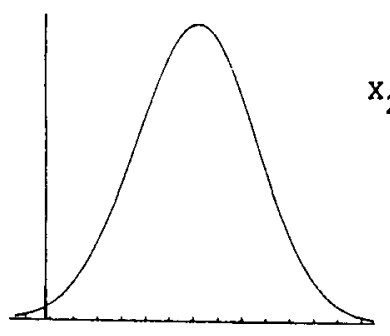

$\mathrm{x}_{2}=$ Normal

$(a=-1.09, b=5.09)$

$(a=-1.09, b=5.09)$
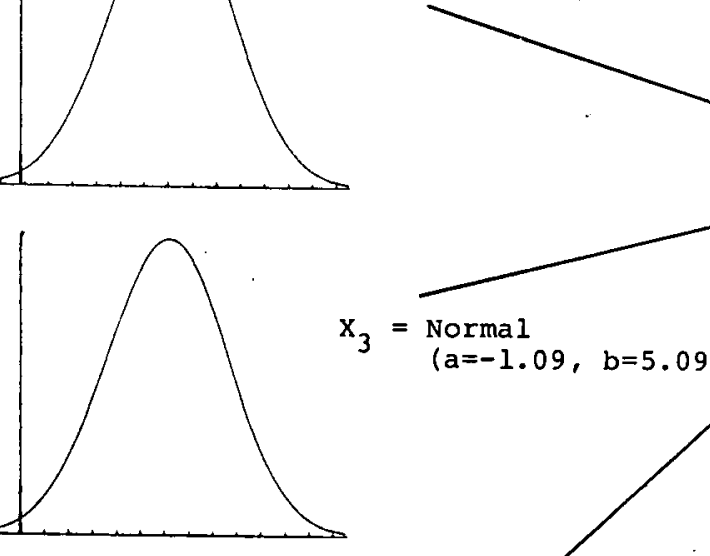

$x_{3}=$ Normal

$(a=-1.09, b=5.09)$

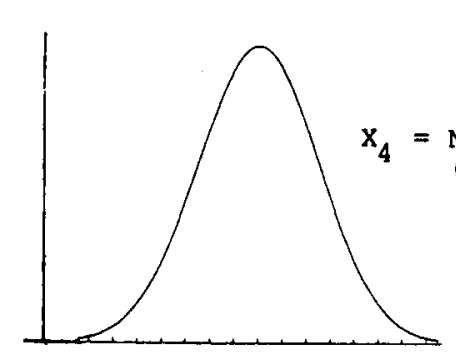

$\mathrm{x}_{4}=$ Normal

$(a=-.09, b=6.09)$

Figure 2. Input Variables and Their Distributions

for a Hypothetical Biack Box Model. 
where there are four input variables and where the input variables have specified probability distributions, all normal in this case. The statistician's job is to understand the desired objectives of the study, and to use (and/or develop) methods for achieving those objectives. Some of the desired objectives of this study are given as follows.

Objectives of a Simulation Study

To assess the probability of the output exceeding specified limits.

For some values of the input variables the output of a model may exceed the limits of acceptability as imposed by regulatory agencies. How likely is: this to happen? Because the input variables have uncertainties associated with them, the exceedance probability can only be estimated on the basis of several runs in which input variables are selected and the output variable is observed. A method for obtaining a confidence interval on this probability is also needed. If several valid methods are available for finding such a confidence interval, the method that gives the smallest interval is obviously the best method to use for achieving this objective.

To determine the sensitivity of the output to the various input variables.

If some input variables are very influential on the output variable, those input variables require close study in any actual site selection decisions. Assumptions regarding the distributions of those variables al so need careful consideration. On the other hand any input variables that show little or no influence on the output variable are not very important to study from a cost effective standpoint, and as sumptions regarding their distributions are not as critical. Statistical methods are needed for measuring the relative importance of the input variables on the basis of several runs of the model. Note that some variables may be important at some time points but not important at other time points, so the method of selecting input values for the various runs of the model should be flexible enough to ailow this determination. The el imination of nonsignificant input variables may result in a substantial simplification of the model, and a sharper focus on the more relevant aspects of the model.

\section{On the Latin Hypercube Sampling Program}

The Latin Hypercube Sampling Program was written to enable a researcher to select input variables according to any of several different methods. It is necessary for the user of this program to specify several items, including the input distributions, the correlation matrix of the input variables, and the type of sampling procedure desired. The program then takes care of obtaining numerical quantities to use as input variables for the model, where those numerical quantities resemble 
values of random variables with the specified probability distributions, with a correlation structure as specified by the user, and selected according to the specified sampling scheme. As the name of the program suggests, one of the options for sampling is Latin Hypercube Sampling, which is a very useful sampling scheme developed specifically for problems such as this one. However, the user may specify Random Sampling instead, which is a frequently used sampling procedure. Variations of these sampling procedures are available as options in this program, however, attention will be focused primarily on these two options.

\section{Options for Input Distributions}

The input distribution is specified separately for each input variable. There are five options available for input distributions; norma 1, uniform, lognormal, loguniform, and a user-specified distribution. The first four distributions are built into the program and are very easy to use. These were discussed earlier. The user-specified distribution requires that a subroutine be written to supply distributions other than those four.

\section{The Distribution Function vs. the Density Function}

Although it is more usual in statistics to think in terms of density functions when describing the distribution of a random variable, there are definite advantages of considering distribution functions (CDFs) in this tutorial. A CDF represents the cumulative probability associated with a random variable. That is, if $f(x)$ is the density function of a continuous random variable $x$, then the distribution function $F(x)$ represents the cumulative probability up to the value $x$,

$$
F(x)=P(x \leqq x)=\int_{-\infty}^{x} f(t) d t
$$

While a random variable must be continuous in order to possess a density function, all random variables possess distribution functions, whether they are continuous, discrete, or some combination of continuous and discrete. Figures $3,4,5$ and 6 illustrate distribution functions and density functions for particular normal, uniform, lognormal, and loguniform distributions. Figure 7 presents the estimated distribution function of the number of boreholes present in a randomly selected 1100 acre tract which is underlain by bedded salt and which has at least one borehole present. Note that this is a discrete distribution function. 
$f(x)$

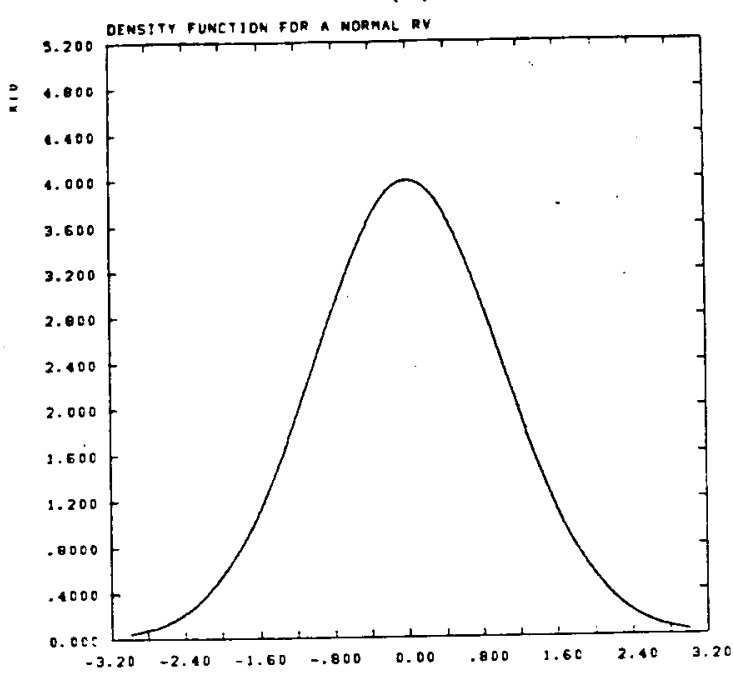

$F(x)$

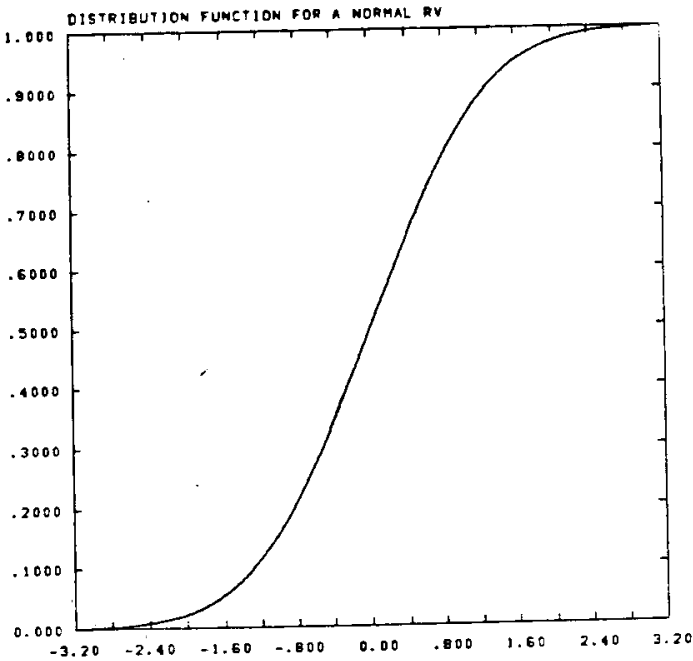

Figure 3. A Normal Density Function $f(x)$ and its Corresponding Distribution Function $F(x)$.
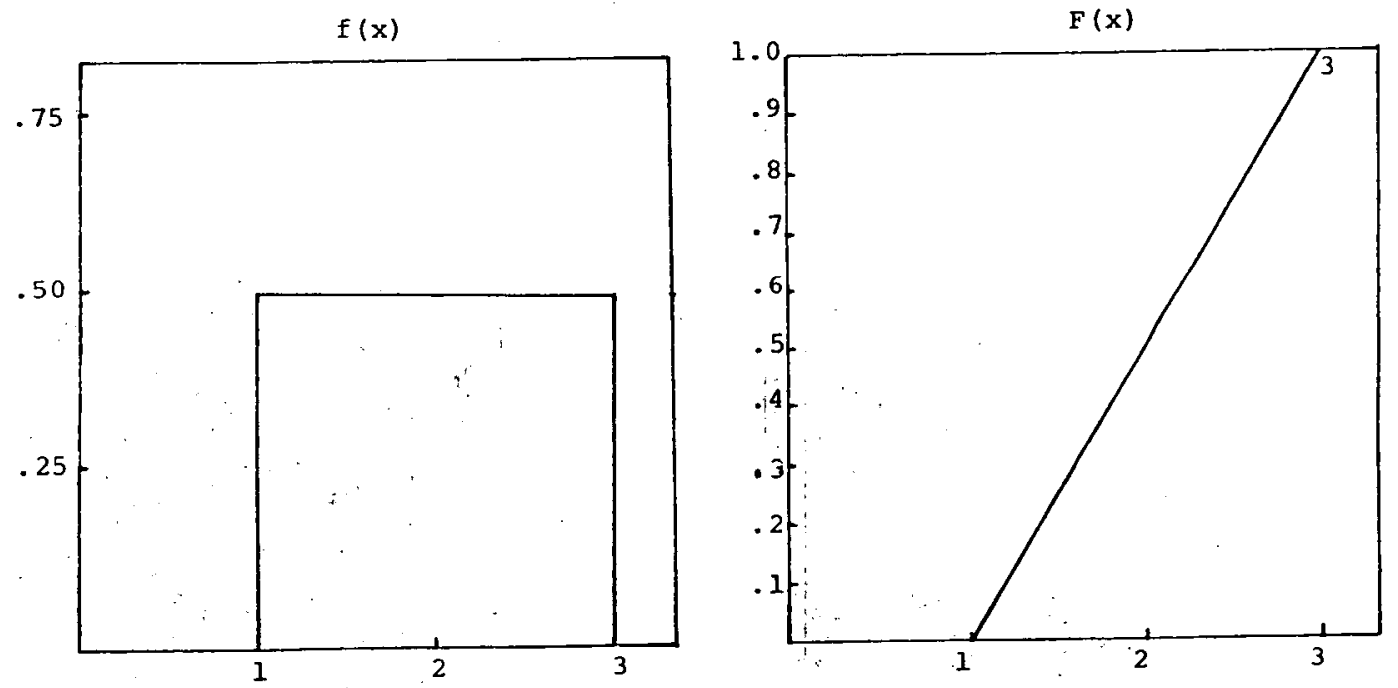

Figure 4. A Uniform Density Function $f(x)$ and its Corresponding Distribution Function $F(x)$.

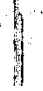


$f(x)$

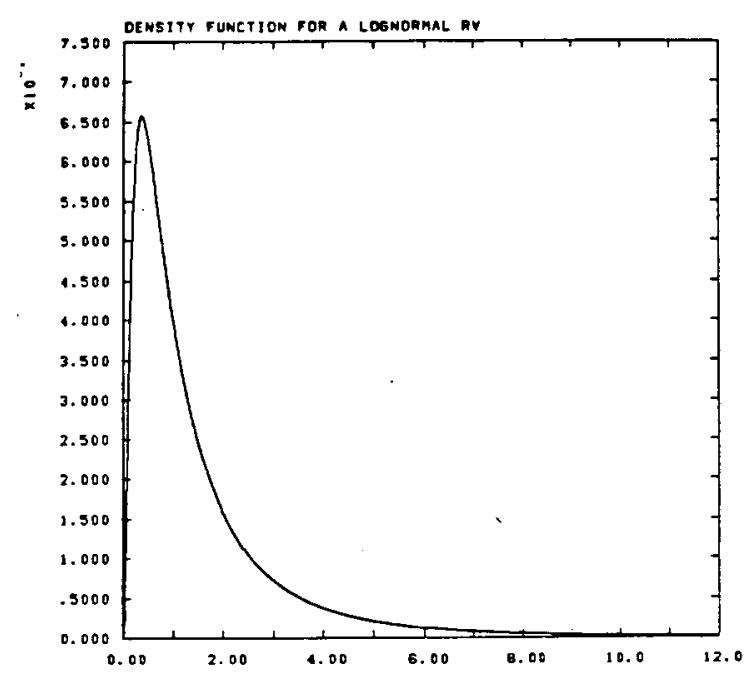

$F(\mathbf{x})$

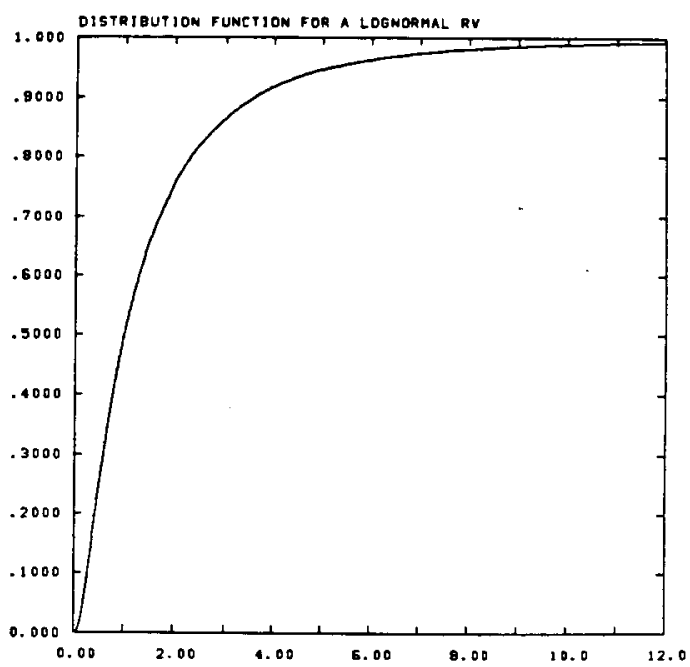

Figure 5. A Lognormal Density Function $f(x)$ and its Corresponding Distribution Function $F(x)$.

$f(x)$

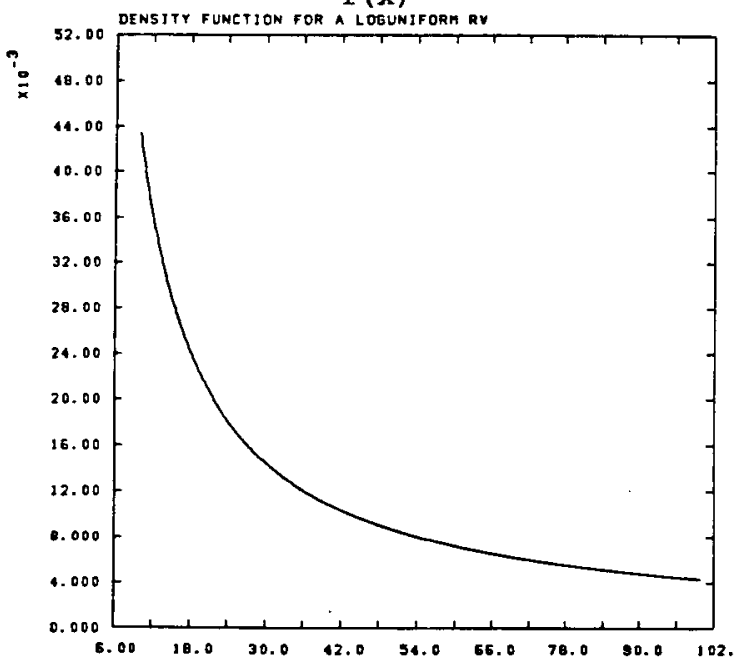

$F(x)$

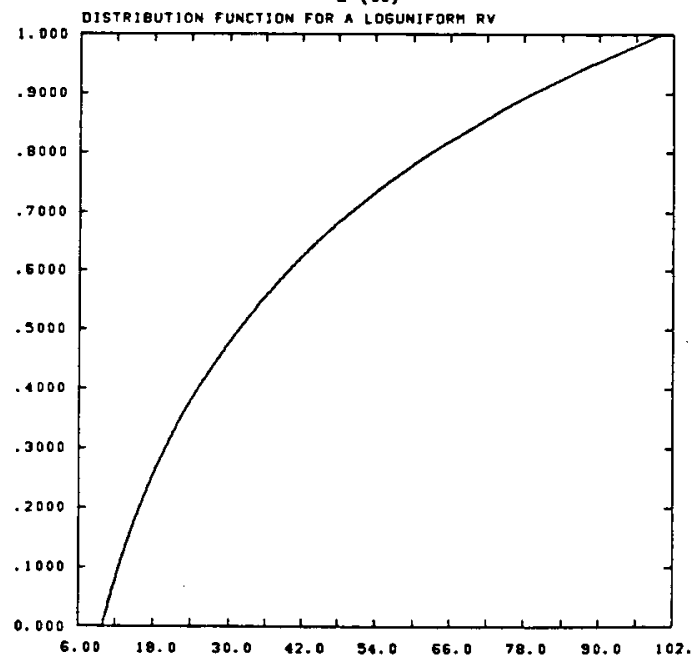

Figure 6. A Loguniform Density Function $f(x)$ and its Corresponding Distribution Function $F(x)$. 
EMPIRICAL DISTRIBUTION FUNCTION FOR

CONDITIONAL DISTRIBUTION OF THE NUMBER

OF BOREHOLES PER 1100 ACRE TRACTS

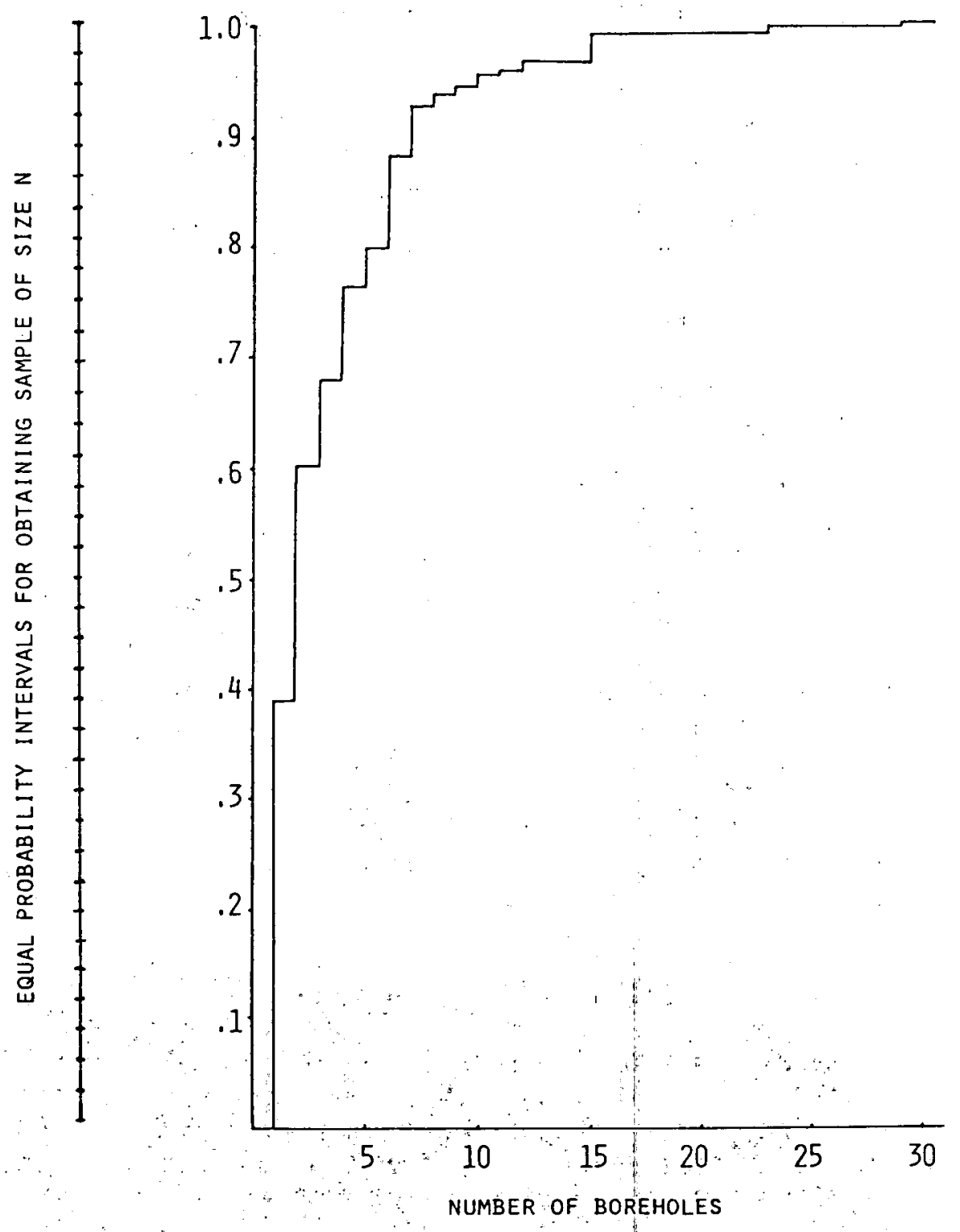

Figure 7., A Distribution Function Estimated From a Large Number of Sample Observations. 
Drawing a Random Sample (I1)ustration)

A hypothetical distribution function is given in Figure 8 for the purposes of illustrating the principles behind drawing a random sample. If a random sample of size $N=2$ is to be drawn from $F(x)$, the following steps are followed.

1. Draw $N=2$ random uniform values. A table of random digits such as Table 1 may be used for this purpose. Enter the table at some randomly selected row (suggestion: let the row number equal the last two digits of your social security number) and a random column (perhaps the third digit from the end of your social security number) and record two sets of numbers, two digits each, reading across. If row 31 , column 3 is selected, the numbers are 87 and 91.

2. Convert these to numbers between 0 and 1 . The simplest way to do this is to divide by 100 , which converts 87 to .87 and 91 to .91 .

3. Use Figure 8 to find $F-1$ for these numbers. The numbers .87 and .91 are found on the vertical axis in Figure 8 , and the inverse function $F^{-1}$ of $F(x)$ is used to convert these to 2.13 and 2.34. These two numbers 2.13 and 2.34 are the random sample of size 2 from the probability distribution given by $F(x)$.

Note that by chance both of the numbers in the random sample in the example happened to be near the upper end of the range of possible values of the random variable being sampled, because the two numbers from Table 1 happened to be close to 100 . This is the nature of random samples; no guarantee is given that the numbers in the sample will be spread out over the range of possible values of the random variable. For this reason, the following method of sampling, called stratified sampling, is often preferred.

Drawing a Stratified Sample (Illustration)

A hypothetical distribution function $F(x)$ (the same one used in Figure 8 ) is given in Figure 9 to illustrate the principle behind stratified sampling. For a stratified sample of size $N=2$, one observation is sampled at random from the lower half (in a probability sense) of the distribution and one value is sampled at random from the upper half.

1. Draw $N=2$ random uniform values. For simplicity the same numbers

87 and 91 will be used again.

2. Convert the first number to a number between 0 and .5 and the second to a number between .5 and 1.0 . First, each number is converted to a number between 0 and .5 by dividing by 200 . Then .5 is added to the second number. 


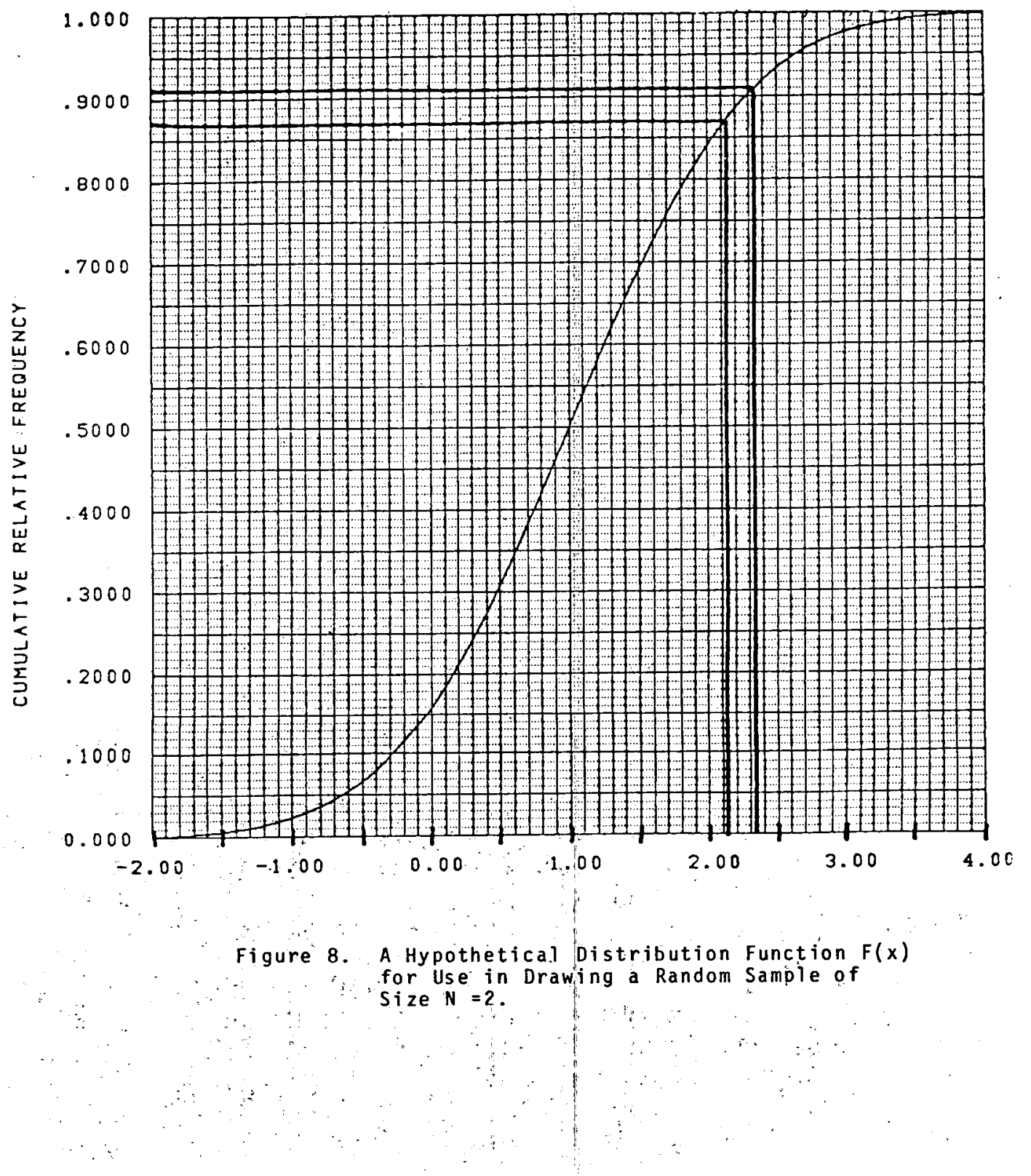




$$
\begin{aligned}
& \frac{87}{200}=.435 \\
& \frac{91}{200}+.5=.455+.5=.955
\end{aligned}
$$

3. Use Figure 9 to find $F^{-1}$ for these numbers. The numbers .435 and .955 are found on the vertical scale in Figure 9 , and the inverse function $F^{-1}$ of $F(x)$ is used to convert these to a stratified sample of size 2 from $F(x)$. The sample consists of the two numbers 0.84 and 2.70. Note that the first number is in the lower half of the distribution and the second number is in the upper half of the distribution, in a probability sense.

For a stratified sample of size $N$, the vertical axis of Figure 9 would be divided into $N$ equal intervals between 0 and 1 , one observation would be sampled at random from each interval using uniform random numbers, and these would be converted to a random sample from $F(x)$ through the use of an inverse function.

More Exact Normal Values from Table 2

The graphical method for finding $x$, given $F(x)$, that was $i 11$ ustrated in the preceding examples is simple and straightforward. It may be used with the graph of any distribution function. The only limitation of such a method is that graphical methods are good to 2 or 3 decimal place accuracy at best. Of course, if the distribution function is discrete, as in Figure 7 , whole integer accuracy may be sufficient.

The distribution function used in the illustrations happens to be normal with mean 1 and variance 1 . Therefore, the exact values for $F^{-1}$, column (d) in Figures 10 and 12, may be found from Table 2. Round the value in column $(c)$ to three decimal places. Enter Table 2 to get $\Phi^{-1}$, the inverse for a standard normal distribution $(\mu=0, \sigma=1)$. Then add 1 to get the inverse for $F(x)$, because $\mu=1$. In general, multiplication by $\sigma$ and addition of $\mu$, in that order, converts from $\Phi^{-1}$ to any normal random variable with mean $\mu$ and variance $\sigma 2$.

The number .87 converts to 1.1264 in Table 2, and then to 2.1264 by adding 1.0. The number .91 converts to 2.3408 using the same procedure, thus justifying the two numbers obtained in the random sample of size 2. For the stratified sample of size 2, the numbers $F(x)=.435$ and $F(x)=.955$ convert to $-.1637+1=.8363$ and 2.6954 respectivel $y$, in agreement with the graphical results but with greater accuracy. 


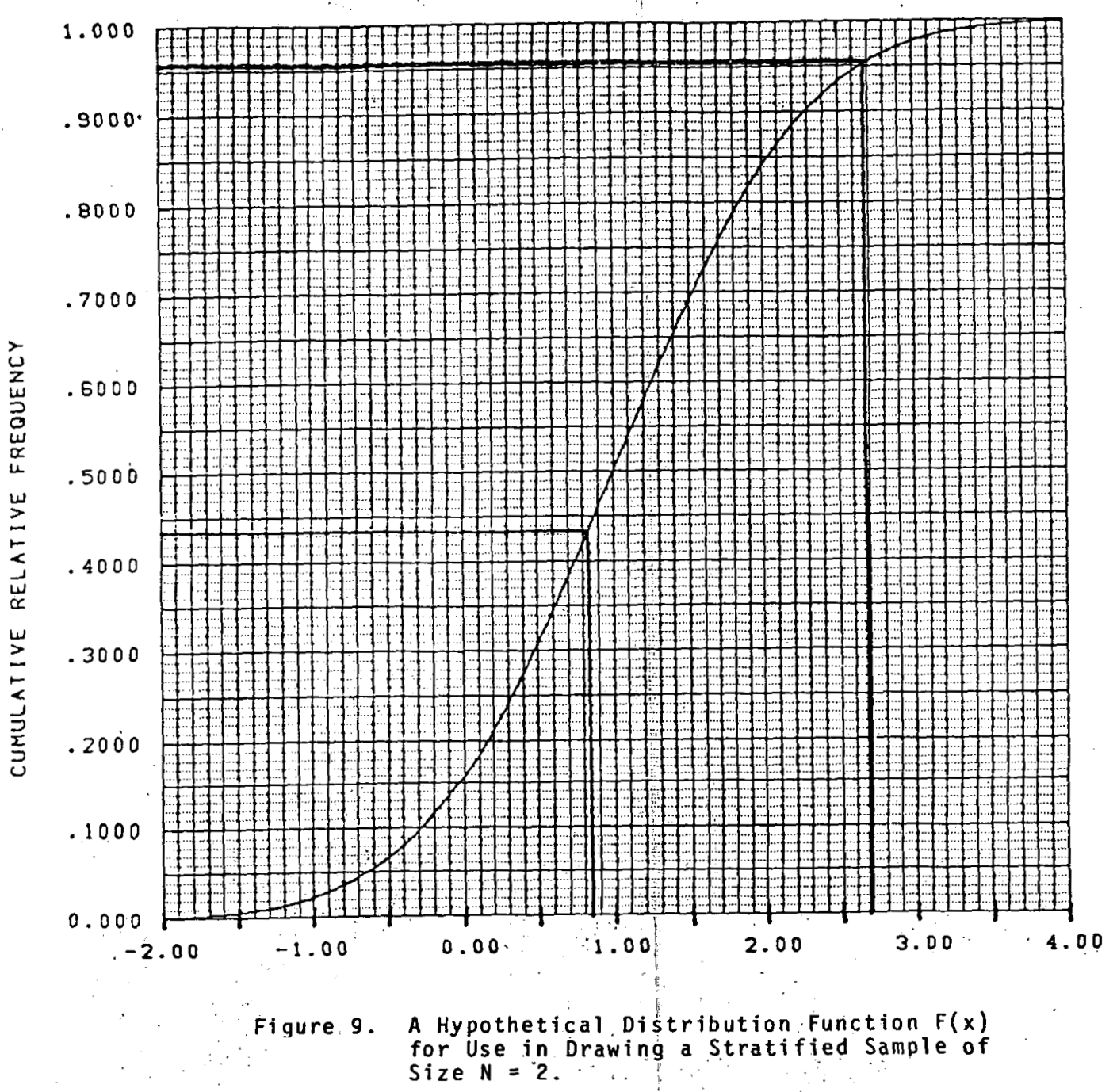




\section{EXERCISE 1}

Use Table 1 and Figures 10 and 11 [columns (a) through (d) only]; to obtain a random sample of size 4 from the distribution $F(x)$.

(a)

(b)

Random

numbers

(2 digits) (c)

$(c)=\frac{(b)}{100}$

(d)

(e)

(f)

$$
F^{-1}(c) \quad \Phi^{-1}(c) \quad(e)+1
$$

1 .

2.

3.

4.

Figure 10. Worksheet for Drawing a Random Sample of Size $N=4$ from $F(x)$. 


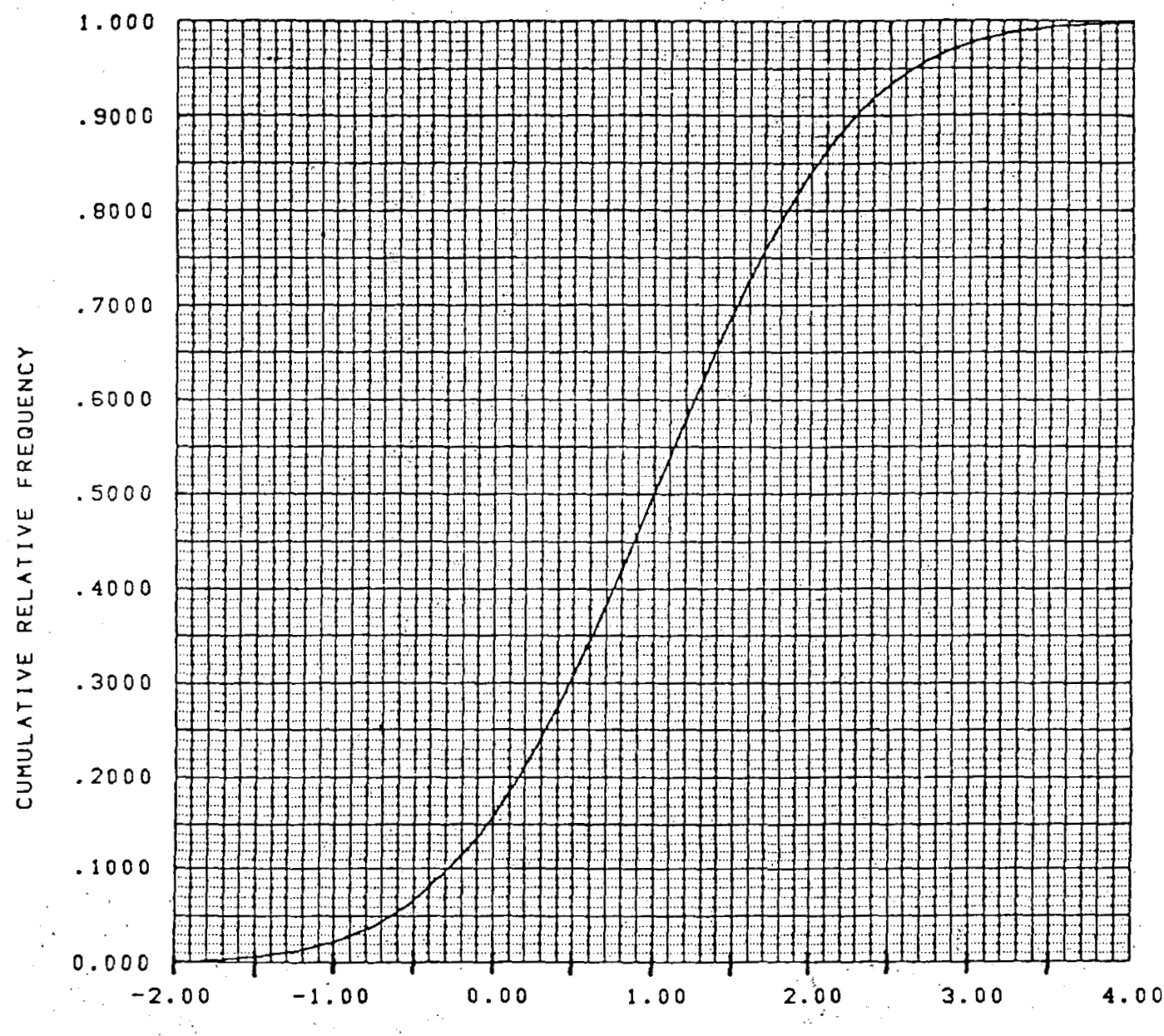

Figure 11. A Hypothetical Distribution Function $F(x)$ for Use in Drawing a Random Sample of size $N=4$. 


\section{EXERCISE 2}

Use Table 1 and Figures 12 and 13 [columns (a) through (d) only], to obtain a stratified sample of size 4 from the distribution $F(x)$. Note how the stratified sample is spread over all four quarters of the probability distribution.
(a)
(b)
(c)
(d)
(e)
(f)
Random
numbers
( 2 digits)
$(c)=\frac{(b)}{400}+\frac{(a)-1}{4}$
$\mathrm{F}^{-1}(\mathrm{c})$
$\Phi^{-1}(c)$
$\Phi^{-1}(c)+1$
1.
2.
3.
4.

Figure 12. Worksheet for Drawing a Latin Hypercube Sample of Size $N=4$ from $F(x)$.

QUESTION 1: For purposes of estimating the mean of the population, do you think the average of a random sample or the average of a stratified sample will tend to give a more accurate figure? ANSWER AT BOTTOM OF PAGE 18. 


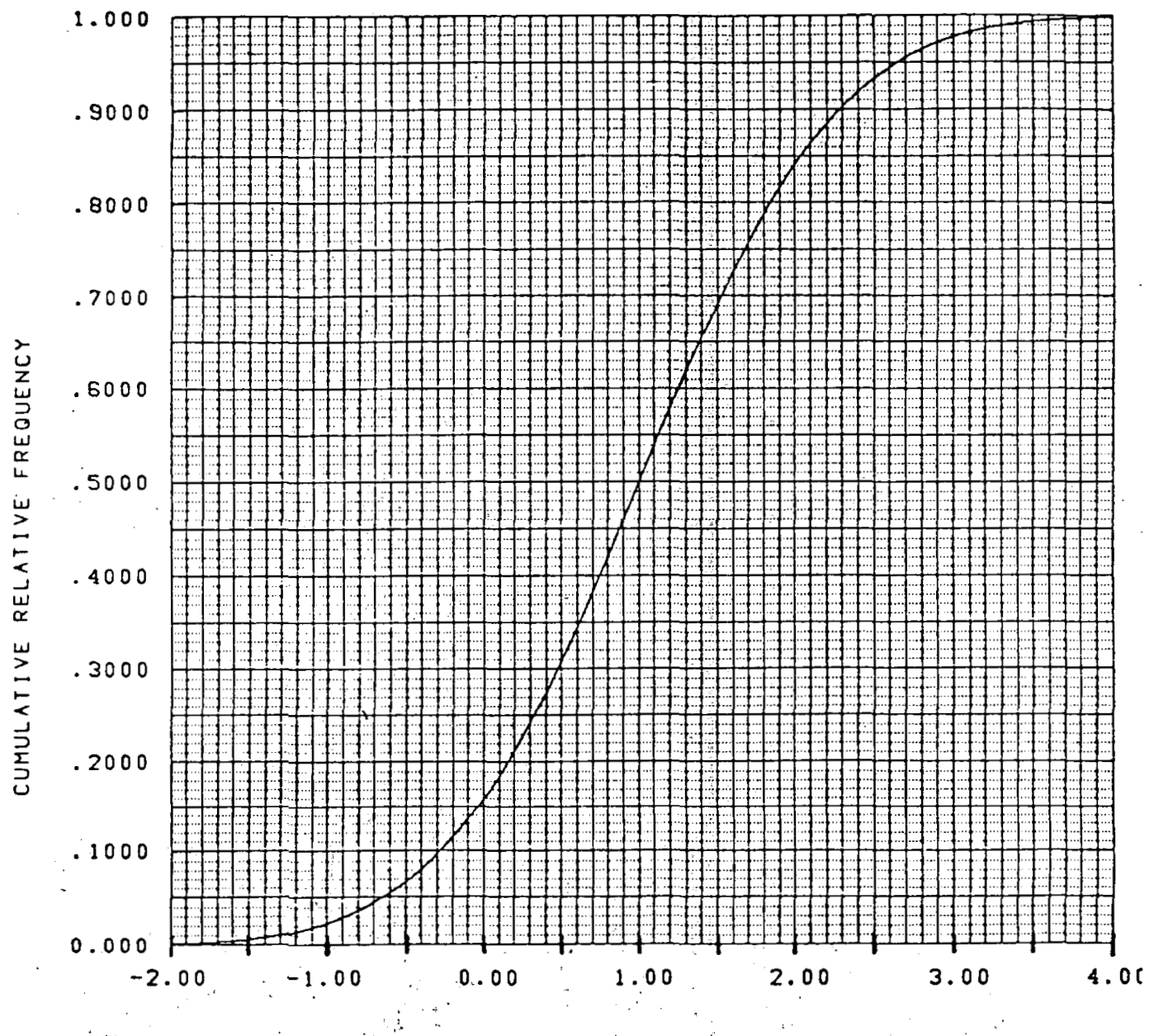

Figure 13. A Hypothetical Distribution Function $F(x)$ for Use in Drawing a Latin Hypercube Sample of Size $N=4$. 


\section{EXERCISE 3}

Use Table 2 and Figure 10, columns (e) and ( $f$ ), to obtain more accurate figures for the random sample from $F(x)$, the normal distribution with $\mu=1$ and $\sigma=1$.

\section{EXERCISE 4}

Use Table 2 and Figure 12, columns (e) and (f), to obtain more accurate figures for the stratified sample from $F(x)$.

ANSWER TO QUESTION 1: It can be shown that the average from a stratified sample will tend to be closer to the true population mean. 
Obtaining a Multivariate Random Sample

The usual model involves many input variables rather than just the one input variable as was used in the previous examples. If four input variables are involved, as in Figure 2 , then one value needs to be obtained for each input variable before an input to the model is complete. Let $K$ be the number of input variables; $K=4$ in Figure 2. Then $K$ numbers are obtained as one input vector, where each number represents one input variable. If $N$ is the number of runs desired, then $\mathrm{N}$ sets of $K$ numbers each are obtained in all.

The Random Sampling method with $K$ input variables is a simple extension of the Random Sampling method for one input variable, if the input variables are independent. The first observation on $x_{1}$ is simply matched with the first observation on $x_{2}$, the first observation on $x_{3}$, and so on, for the first input vector. The second input vector consists of the second values obtained for $x_{1}, x_{2}, \ldots, x_{K}$, and so on for all $\mathrm{N}$ input vectors. The situation is not so simple if some specified correlation is desired on the input values, but that will be described later.

\section{Obtaining a Latin Hypercube Sample}

To obtain a Latin Hypercube Sample, when the input variables are uncorrelated, the situation is almost as simple. First, stratified samples of size $\mathrm{N}$ are obtained on each input variable, in the manner previously described for finding stratified samples. Then the stratified sample of size $N$ on $X_{1}$ is permuted into a random order, using some randomization method. The $N$ observations on $X_{2}$ are also permuted into a random order, independent of the order on the values of $x_{1}$. The values for each input variable are arranged in a random order, independent of the order of the other input variables.

Once the samples are permuted as described, the Latin Hypercube Sample is easily constructed. The first value of $x_{1}$ is matched with the first values of $x_{2}, x_{3}, \ldots, x_{k}$ for the first input vector. The second values of each are matched for the second input vector. This matching procedure is followed until all $\mathrm{N}$ values of each variable are used. The method for matching the observations to achieve some target correlation values will be given later, for the non-independent case.

Comparing Latin Hypercube with Random Sampling

A Latin Hypercube Sample has observations that are spread over the entire range of each input variable, and the spread is in a uniform manner, in a probability sense. This is unlike the Random Sample which may produce clusters of observations anywhere in the range of the variables. It is difficult to prove analytically that either method of sampling is better than the other. However, some comparisons of the two sampling methods have been made with actual models, and the Latin 
Hypercube Samples appear to give much better results where the goal is to estimate the distribution function of the output variable. The following exercises are designed to illustrate how to obtain Random Samples and Latin Hypercube Samples with multivariate input, when the input variables are uncorrelated. Then comparisons will be made between the two methods to see what kind of accuracy is obtained on estimates of the output.

Obtaining a Multivariate Random Sample (Illustration)

Refer to Figure 2 where there are four input variables each with a normal distribution function. In each run observations on these four input variables go into a model, represented by a black box, and the output is recorded. The following exercise takes the reader step by step through the process of obtaining a random sample of size 10 . The subsequent exercise takes the reader through the steps in obtaining a Latin Hypercube Sample of size 10. Later these samples will be entered into a black-box type model and the outputs recorded and compared. But first the samples are obtained.

\section{EXERCISE 5 (Multivariate random sample of size 10)}

1. In order to draw a 4-variate random sample of size 10,40 random numbers are needed from Table 1. Starting where you left off in Exercise 2, choose 40 three-digit random numbers, reading across the table row by row. Record these in the order drawn, down column (b) in Figures 14-17.

2. The numbers in column (b) are converted to probabilities between 0 and 1 by dividing by 1000, for column (c).

3. The probabilities in column (c) are converted to random samples from a standard normal distribution $(\mu=0, \sigma=1)$ by entering Table 2 in the respective row and column, and recording the table entry in column (d).

4. The standard normal values from column (d) are converted to normal values with other means by adding the constant indicated in column (e) of each figure.

5. Transcribe all 40 numbers in column (e) into the respective columns of Figure 18. Each row of Figure 18 represents one input to the black box model. 


$$
\begin{aligned}
& (a) \\
& \text { Obs. } \\
& \text { Numbe } \\
& 1 . \\
& 2 . \\
& 3 . \\
& 4 . \\
& 5 . \\
& 6 . \\
& 7 . \\
& 8 . \\
& 9 . \\
& 9 .
\end{aligned}
$$

Number

(b)

Uni form

Random

Number

(3 digits) (c)

(b) $\Phi^{-1}(c)$

$(c)=\frac{(b)}{1000}$ from Table 2 (e)

Input

$(e)=(d)+1$

Figure 14. Worksheet for Drawing a Random Sample of Size 10 for $x_{1}$ where $x_{1}$ is Normal, $\mu=1, \sigma=1$. 
(a)

Obs.

Number (b)

Uniform

Random

Number

(3 digits) (c)

$(c)=\frac{(b)}{1000}$ (d)

$\Phi^{-1}(c)$

from Table 2 (e)

Input

$(e)=(d)+2$

1.

2.

3.

4.

5.

6.

7.

8.

9.

10.

Figure 15. Worksheet for Drawing a Random Sample of Size 10 for $X_{2}$, where $x_{2}$ is Normal, $\mu=2, \sigma=1$. 
(a)

Obs.

Number (b)

Uni form

Random

Number (c)

(c) $=\frac{(b)}{1000}$ (d)

$\Phi^{-1}(c)$ (e)

$(e)=(d)+2$

1.

2.

3.

4.

5.

6.

7.

8.

9.

10.

Figure 16. Worksheet for Drawing a Random Sample of Size 10 for $X_{3}$, where $X_{3}$ is Normal, $\mu=2, \sigma=1$. 


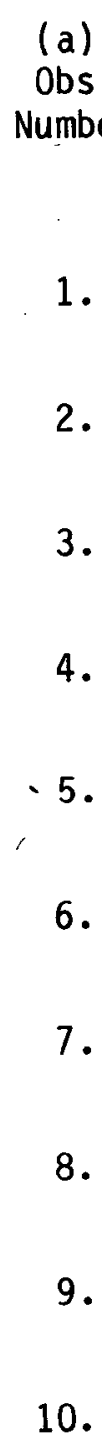

(b)

Uniform

Random

Number (c)

$(c)=\frac{(b)}{1000} \quad \Phi^{-1}(c)$ (e)

$(e)=(d)+3$

1.

2.

3.

4.

5.

6.

7.

8.

9.

10.

Figure 17. Worksheet for Drawing a Random Sample of Size 10 for $X_{4}$, where $x_{4}$ is Normal, $\mu=3, \sigma=1$. 
6. Using a pre-programmed calculator, or the equation

$$
y=x_{1}+x_{2} x_{3}-x_{2} \ln \left|x_{1}\right|+\exp \left(x_{4} / 4\right)
$$

whichever is more convenient, find the output value $Y$ for each of the 10 input values $\left(x_{1}, x_{2}, x_{3}, x_{4}\right)$, and record these in column (f) of Figure 18.

7. Plot the 10 values of $Y$ on the abscissa of Figure 19, and draw an empirical distribution function. An empirical distribution function is a step function which equals zero on the left, and proceeding from left to right, rises a height of $1 / \mathrm{N}$ at each of the $\mathrm{N}$ sample points, until it equals 1.0. This is an estimate of the true distribution function of the output. In this case, start at zero on the left and increase the height of the graph by $1 / 10$ at each observation on $Y$, as the graph proceeds from left to right. At the largest observed value of $Y$ the graph should jump from a height of .9 to a height of 1.0 .

The Accuracy of the Output From a Random Sample

The empirical distribution function in Figure 19 provides an estimate of the population distribution function of the output. The sample mean of the 10 values of $Y$ provides an estimate of the population mean, and other sample values provide estimates of their corresponding population values in the usual manner for random samples.

In order to see how well these samples function as the basis for population estimates, five random samples of size 10 each were obtained using the Latin Hypercube Sampling Program, and were entered into the black box model to obtain outputs. The five empirical distribution functions are given in Figure 20, while Figure 21 presents a picture of the mean of all five e.d.f.'s together. In the background of Figures 20 and 21 is an accurate estimate of the true distribution function of the output, obtained by using a random sample of size $N=1000$.

The mean of all five e.d.f.'s, averaged in the vertical direction, is plotted again in Figure 22. This is the same e.d.f. one would obtain if all 50 sample observations were treated as a random sample of size $N=50$, which it actually is. Above and bel ow the mean curve. in Figure 22 are curves that represent one standard deviation distance, where the standard deviation is computed vertically from the five curves in Figure 20 , and smoothed using a three point moving average. The standard deviation is presented to givel some idea of the accuracy involved in each individual random sample of size 10 . 


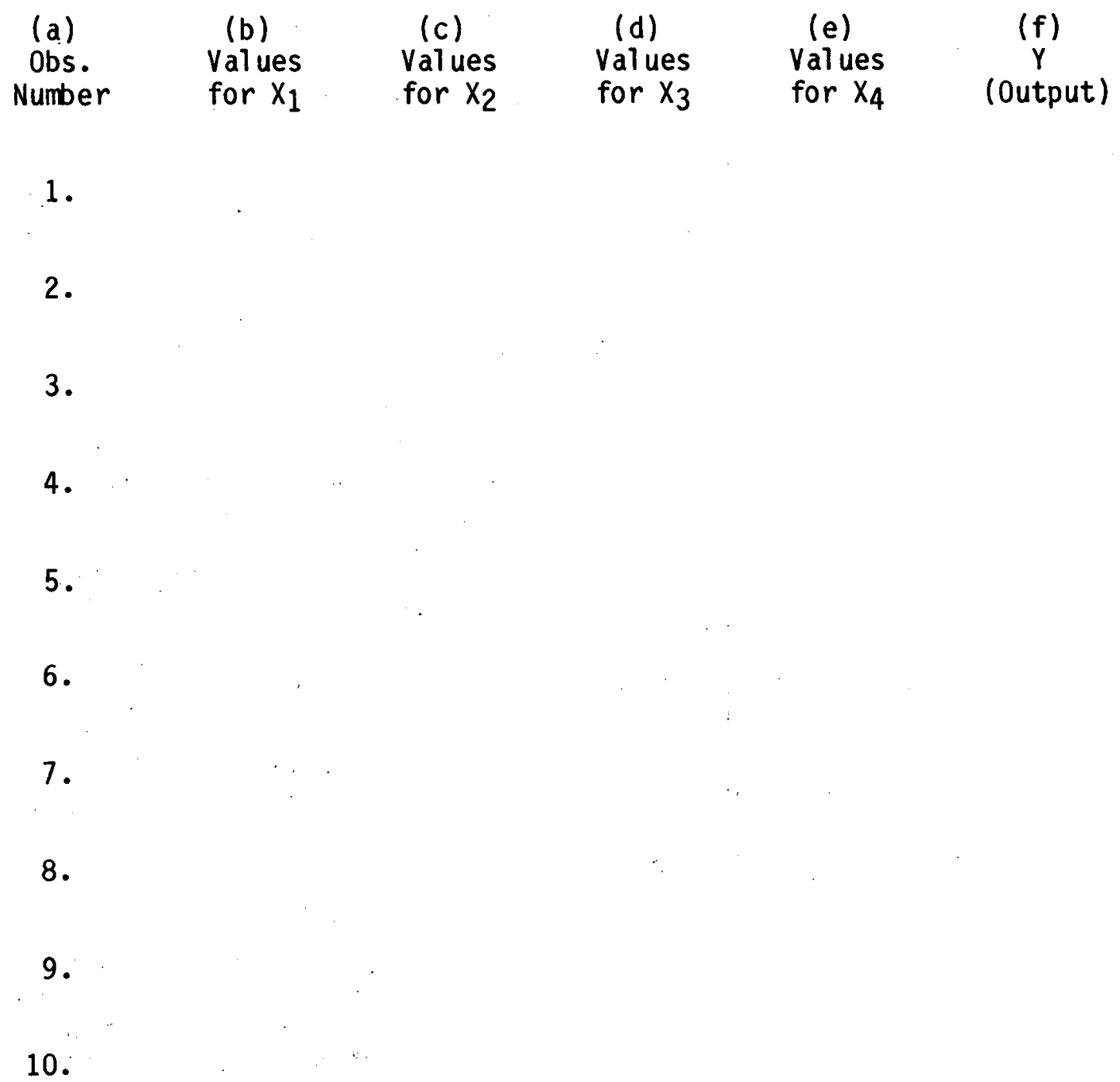

Figure 18. The Multivariate Input Vectors Using a Random Sample, and the Corresponding Output. 


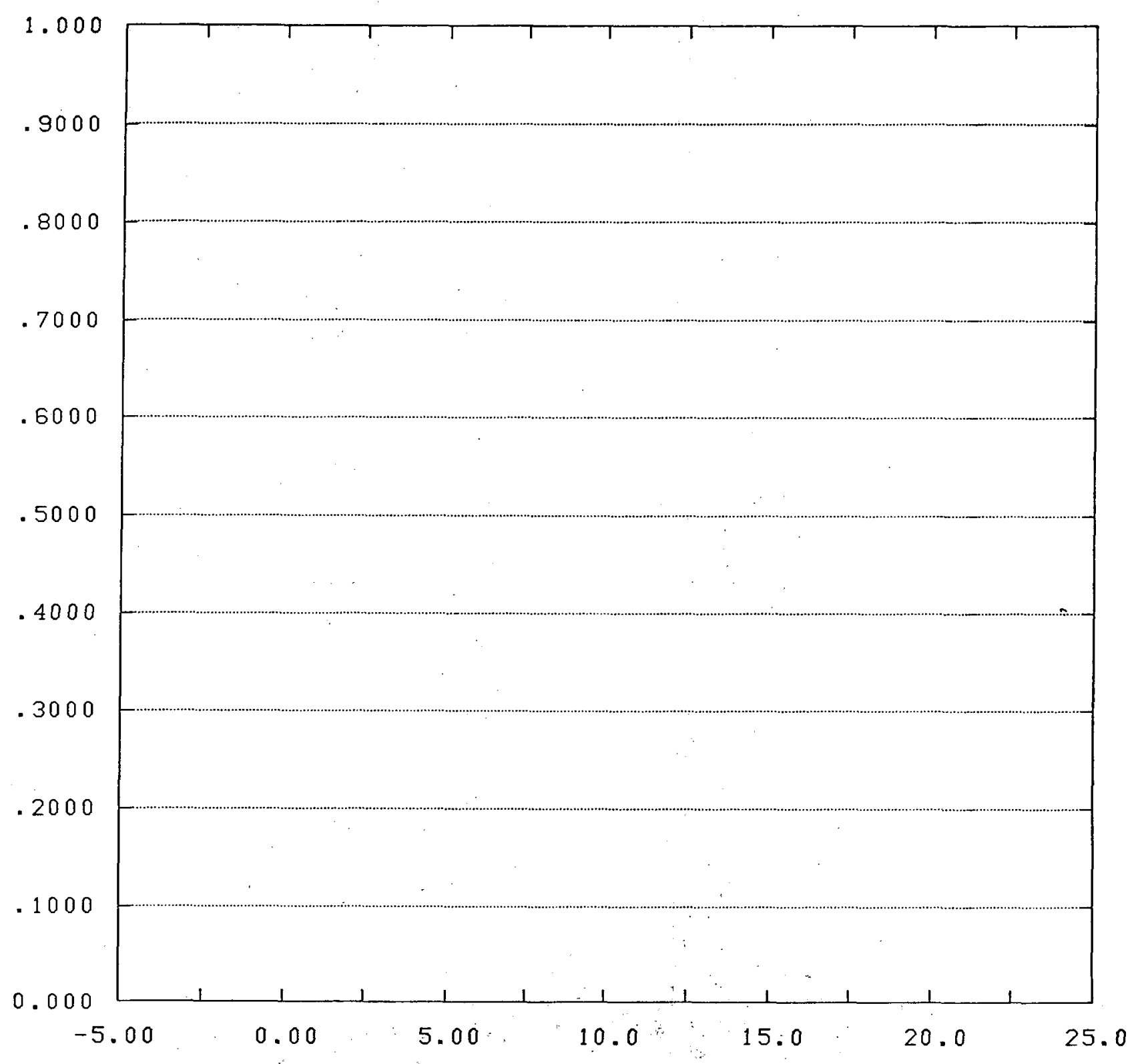

Figure 19. An Empirical Distribution Function from the Random Sample of Figure 18. 
b)

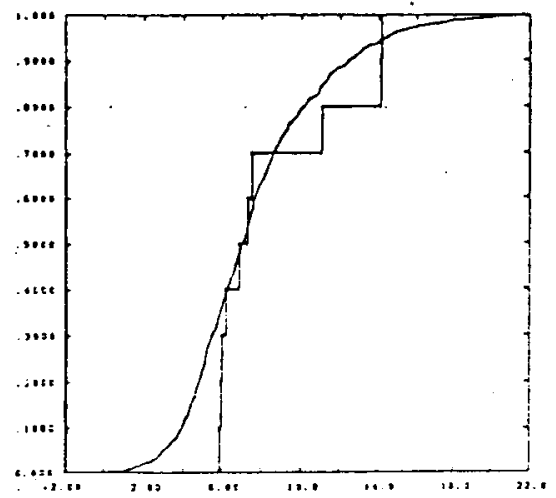

(c)

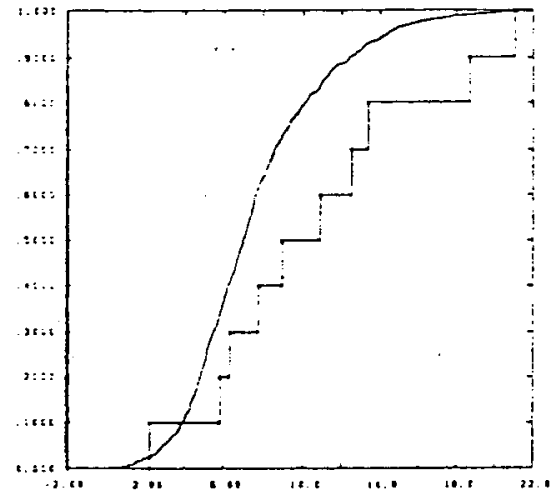

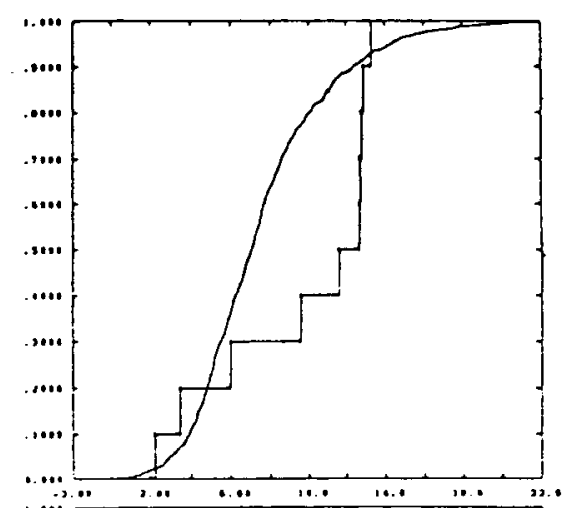

(b)

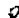

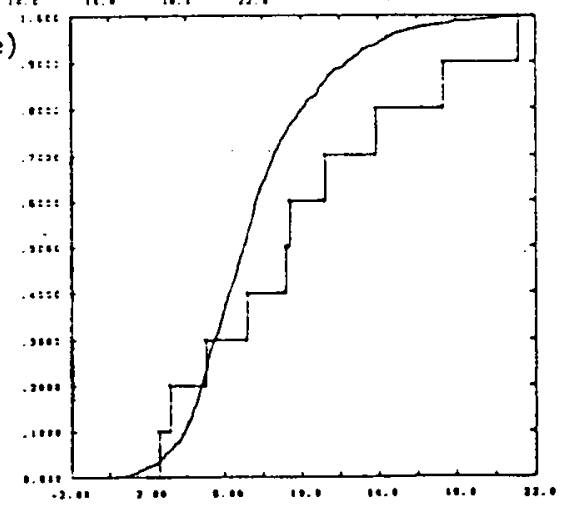

Figure 20. Five Empirical Distribution Function (a) - (e) from Random Samples of Size 10, and an Estimate of the Population Distribution Function 
RS INDEP. SAMP NRML VS RS INDEP SAMP NRML $N=50$

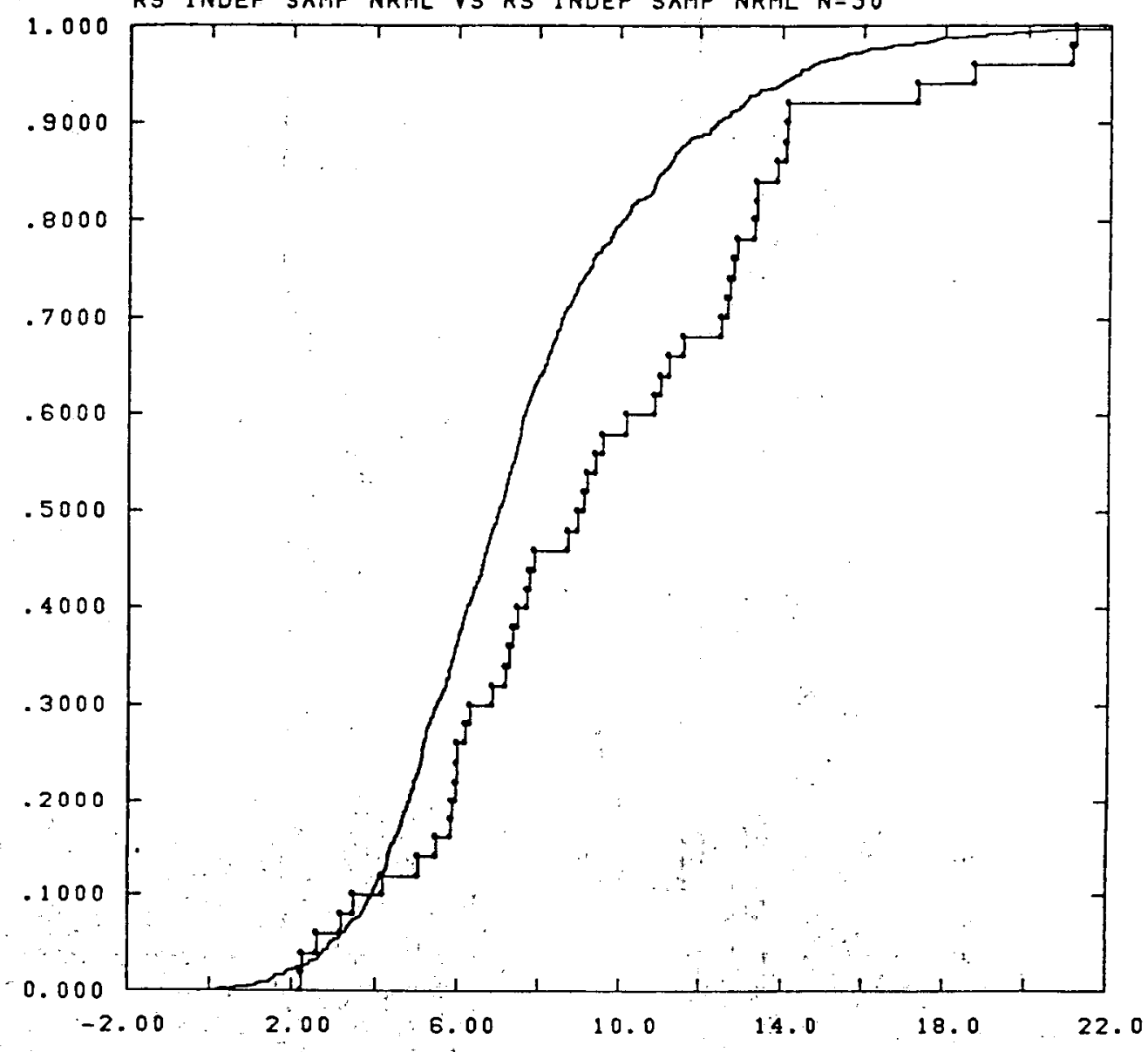

Figure 21.: A Graph of the Mean of the Five EDF's from Figure 20, and an Estimate of the Population Distribution Function 


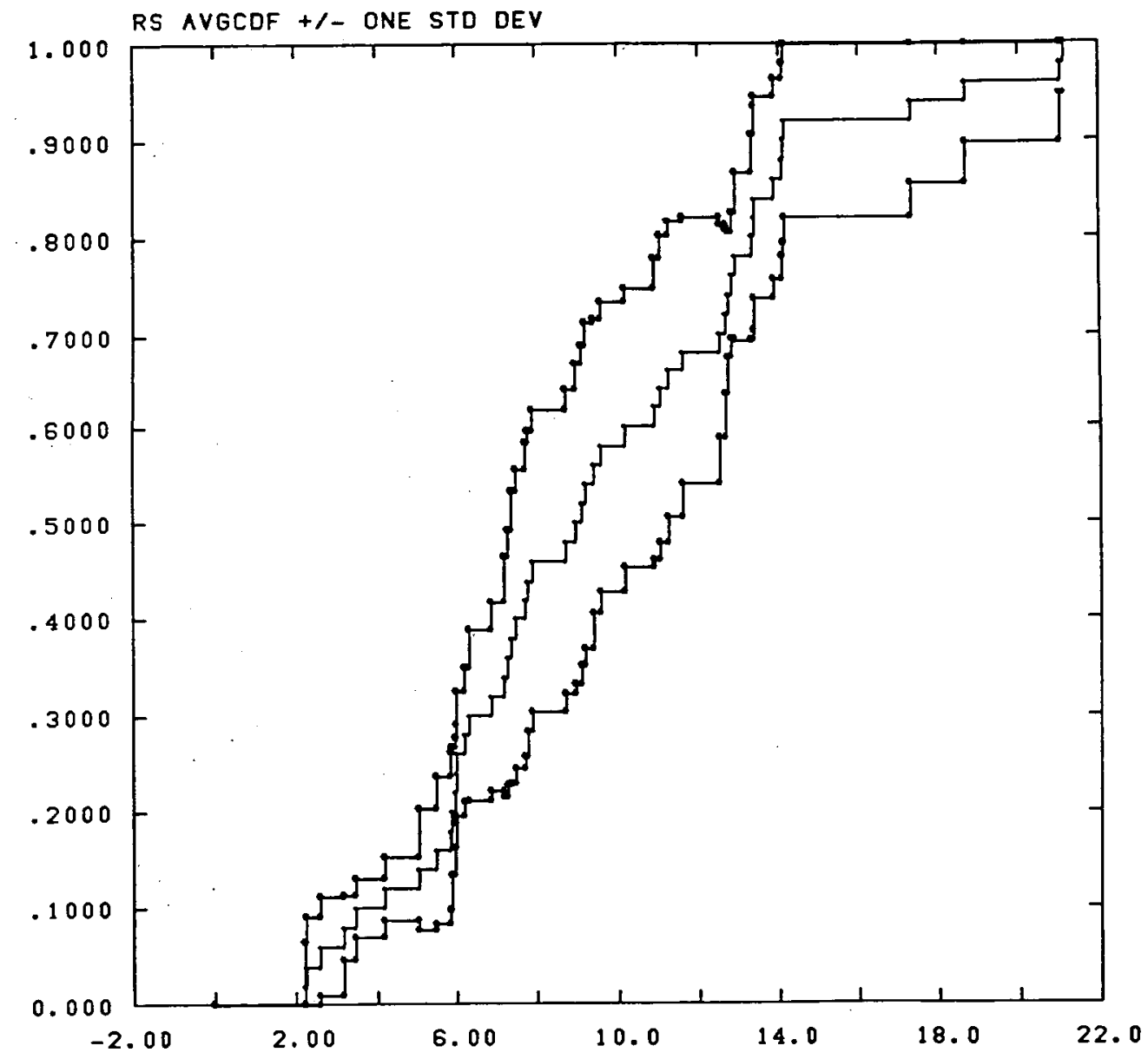

Figure 22. The Mean and One Standard Deviation Bounds of the Five EDFs from Figure 20. 
Obtaining a Random Permutation

Before drawing a Latin Hypercube Sample, one method for arranging numbers in a random order will be discussed. One way of obtaining a random permutation of the integers 1 to $N$ is to draw $N$ numbers from a random number table (or a computer program) and use the ranks of those numbers as the random permutation. For example, starting with row 41 , column 1 in Table 1, ten consecutive 4-digit numbers are given as follows, along with their ranks.

$\begin{array}{lr}\begin{array}{lr}\text { Random } \\ \text { Numbers }\end{array} & \text { Ranks } \\ 9 & 10 \\ 9842 & 5 \\ 7075 & 2 \\ 2333 & 3 \\ 3626 & 4 \\ 4270 & 1 \\ 0163 & 8 \\ 8924 & 6 \\ 7766 & 9 \\ 9699 & 7 \\ 8420 & \end{array}$

Since the random numbers fol low a random ordering, the ranks form a random permutation of the integers from 1 to 10 .

Obtaining a Latin Hypercube Sample (Illustration)

The following steps outline the procedure for finding a Latin Hypercube Sample. The situation described in Figure 2 is used. A sample of size $N=10$ will be formed using $K=4$ input variables, which are independent of each other, and normaliy distributed with $\sigma^{2}=1$ and means $\mu_{1}=1, \mu_{2}=2, \mu_{3}=2, \mu_{4}=3$ respectively. The reader should follow through the steps, and Figures 23-28 to ensure an understanding of the process.

Step 1. Obtain uniform random numbers in each of $N$ strata. Select a random starting point in Table 1 and, reading across, select 40 twodigit random numbers. Write these in column (b) in Figures 23-26. Divide each of these numbers by 1000 and add the lower bound for the strata from column (a): Put the result in column (c). These are the stratified sample values of $F(x)$.

Step 2. Arrange the values of $F(x)$ in a random order. Draw an additional 40 random numbers from Table 2 , using 4 -digit numbers to reduce the chances of ties and put these numbers in column (d). In the case of ties, redraw until there are no ties. Rank each 10 of these from 1 to 10 in each Figure and put the ranks in column (e). 


\begin{tabular}{|c|c|c|c|c|c|c|c|}
\hline $\begin{array}{l}\text { (a) } \\
\text { Lower } \\
\text { Bound } \\
\text { of } \\
\text { Stratum }\end{array}$ & $\begin{array}{l}\text { (b) } \\
\text { Uni form } \\
\text { Random } \\
\text { Numbers }\end{array}$ & $F(x)$ & $\begin{array}{l}\quad(d) \\
\text { Uni form } \\
\text { Random } \\
\text { Numbers } \\
\end{array}$ & $\begin{array}{c}\text { (e) } \\
\text { Ranks } \\
\text { of } \\
\text { (d) } \\
\end{array}$ & $\begin{array}{l}(f) \\
\text { Random } \\
\text { Order } \\
\text { of } F(x) \\
\end{array}$ & $\begin{array}{c}\text { (g) } \\
\Phi^{-1}\end{array}$ & $\begin{array}{c}(h) \\
F^{-1}=(g)+1\end{array}$ \\
\hline $\begin{array}{l}.0 \\
.1 \\
.2 \\
.3 \\
.4 \\
.5 \\
.6 \\
.7 \\
.8 \\
.9\end{array}$ & $\begin{array}{l}31 \\
93 \\
52 \\
70 \\
22 \\
84 \\
06 \\
70 \\
54 \\
62\end{array}$ & $\begin{array}{l}.031 \\
.193 \\
.252 \\
.370 \\
.422 \\
.584 \\
.606 \\
.770 \\
.854 \\
.962\end{array}$ & $\begin{array}{l}7216 \\
3095 \\
3812 \\
1510 \\
6878 \\
9190 \\
3187 \\
4934 \\
4055 \\
6087\end{array}$ & $\begin{array}{r}9 \\
2 \\
4 \\
1 \\
8 \\
10 \\
3 \\
6 \\
5 \\
7\end{array}$ & $\begin{array}{l}.854 \\
.193 \\
.370 \\
.031 \\
.770 \\
.962 \\
.252 \\
.584 \\
.422 \\
.606\end{array}$ & $\begin{array}{r}1.05 \\
-0.87 \\
-0.33 \\
-1.87 \\
0.74 \\
1.77 \\
-0.67 \\
0.21 \\
-0.20 \\
0.27\end{array}$ & $\begin{array}{r}2.05 \\
0.13 \\
0.67 \\
-0.87 \\
1.74 \\
2.77 \\
0.33 \\
1.21 \\
0.80 \\
1.27\end{array}$ \\
\hline
\end{tabular}

Figure 23. A Stratified Sample of Size 10 for $X_{1}$ from a Normal Population with $\mu=1$ and $\sigma^{2}=1$.

\begin{tabular}{|c|c|c|c|c|c|c|c|}
\hline $\begin{array}{l}\text { (a) } \\
\text { Lower } \\
\text { Bound } \\
\text { of } \\
\text { Stratum }\end{array}$ & $\begin{array}{l}\text { (b) } \\
\text { Uniform } \\
\text { Random } \\
\text { Numbers }\end{array}$ & $F(x)$ & $\begin{array}{l}\quad \text { (d) } \\
\text { Uniform } \\
\text { Random } \\
\text { Numbers }\end{array}$ & $\begin{array}{l}\text { (e) } \\
\text { Ranks } \\
\text { of } \\
\text { (d) }\end{array}$ & $\begin{array}{l}(f) \\
\text { Random } \\
\text { Order } \\
\text { of } F(x)\end{array}$ & $\begin{array}{l}(g) \\
\Phi^{-1}\end{array}$ & $\begin{array}{c}(h) \\
F^{-1}=(g)+2\end{array}$ \\
\hline $\begin{array}{l}.0 \\
.1 \\
.2 \\
.3 \\
.4 \\
.5 \\
.6 \\
.7 \\
.8 \\
.9\end{array}$ & $\begin{array}{l}35 \\
21 \\
61 \\
44 \\
86 \\
29 \\
89 \\
16 \\
86 \\
07\end{array}$ & $\begin{array}{r}.035 \\
.121 \\
.261 \\
.344 \\
.486 \\
.529 \\
.689 \\
.716 \\
.886 \\
.907\end{array}$ & $\begin{array}{l}8279 \\
0709 \\
2565 \\
3900 \\
5224 \\
7295 \\
8286 \\
0981 \\
4063 \\
9147\end{array}$ & $\begin{array}{r}8 \\
1 \\
3 \\
4 \\
6 \\
7 \\
9 \\
2 \\
5 \\
10\end{array}$ & $\begin{array}{l}.716 \\
.035 \\
.261 \\
.344 \\
.529 \\
.689 \\
.886 \\
.121 \\
.486 \\
.907\end{array}$ & $\begin{array}{r}0.57 \\
-1.81 \\
-0.64 \\
-0.40 \\
0.07 \\
0.49 \\
1.21 \\
-1.17 \\
-0.04 \\
1.32\end{array}$ & $\begin{array}{l}2.57 \\
0.19 \\
1.36 \\
1.60 \\
2.07 \\
2.49 \\
3.21 \\
0.83 \\
1.96 \\
3.32\end{array}$ \\
\hline
\end{tabular}

Figure 24. A Stratified Sample of Size 10 for $X_{2}$ from a Normal Population with $\mu=2$ and $\sigma^{2}=1$. 
(a)

\begin{tabular}{|c|c|c|c|c|c|c|c|}
\hline $\begin{array}{l}\text { Lower } \\
\text { Bound } \\
\text { of } \\
\text { Stratum }\end{array}$ & $\begin{array}{l}\quad \text { (b) } \\
\text { Uni form } \\
\text { Random } \\
\text { Numbers }\end{array}$ & $\begin{array}{l}(c) \\
F(x)\end{array}$ & $\begin{array}{l}\quad(d) \\
\text { Uni form } \\
\text { Random } \\
\text { Numbers }\end{array}$ & $\begin{array}{l}\text { (e) } \\
\text { Ranks } \\
\text { of } \\
\text { (d) }\end{array}$ & $\begin{array}{l}(f) \\
\text { Random } \\
\text { Order } \\
\text { of } F(x)\end{array}$ & $\begin{array}{l}(g) \\
\Phi^{-1}\end{array}$ & $\begin{array}{c}(h) \\
F^{-1}=(g)+2\end{array}$ \\
\hline $\begin{array}{l}.0 \\
.1 \\
.2 \\
.3 \\
.4 \\
.5 \\
.6 \\
.7 \\
.8 \\
.9\end{array}$ & $\begin{array}{l}41 \\
86 \\
71 \\
49 \\
51 \\
91 \\
69 \\
68 \\
50 \\
65\end{array}$ & $\begin{array}{l}.041 \\
.186 \\
.271 \\
.349 \\
.451 \\
.591 \\
.669 \\
.768 \\
.850 \\
.965\end{array}$ & $\begin{array}{l}2554 \\
9485 \\
4242 \\
6274 \\
5233 \\
5720 \\
2946 \\
1723 \\
7720 \\
7896\end{array}$ & $\begin{array}{r}2 \\
10 \\
4 \\
7 \\
5 \\
6 \\
3 \\
1 \\
8 \\
9\end{array}$ & $\begin{array}{l}.186 \\
.965 \\
.349 \\
.669 \\
.451 \\
.591 \\
.271 \\
.041 \\
.768 \\
.850\end{array}$ & $\begin{array}{r}-0.89 \\
1.81 \\
-0.39 \\
0.44 \\
-0.12 \\
0.23 \\
-0.61 \\
-1.74 \\
0.73 \\
1.04\end{array}$ & $\begin{array}{l}1.11 \\
3.81 \\
1.61 \\
2.44 \\
1.88 \\
2.23 \\
1.39 \\
0.26 \\
2.73 \\
3.04\end{array}$ \\
\hline
\end{tabular}

Figure 25. A Stratified Sample of Size 10 for $X_{3}$ from a Normal Population with $\mu=2$ and $\sigma^{2}=1$.

(a)

\begin{tabular}{|c|c|c|c|c|c|c|c|}
\hline $\begin{array}{l}\text { Lower } \\
\text { Bound } \\
\text { of } \\
\text { Stratum }\end{array}$ & $\begin{array}{l}\quad \text { (b) } \\
\text { Uni form } \\
\text { Random } \\
\text { Numbers }\end{array}$ & (c) & $\begin{array}{l}\quad(d) \\
\text { Uni form } \\
\text { Random } \\
\text { Numbers } \\
\end{array}$ & $\begin{array}{l}\text { (e) } \\
\text { Ranks } \\
\text { of } \\
\text { (d) } \\
\end{array}$ & $\begin{array}{l}\text { (f) } \\
\text { Random } \\
\text { Order } \\
\text { of } F(x) \\
\end{array}$ & $\begin{array}{l}(g) \\
\Phi^{-1}\end{array}$ & $\begin{array}{c}(h) \\
F^{-1}=(g)+3\end{array}$ \\
\hline $\begin{array}{l}.0 \\
.1 \\
.2 \\
.3 \\
.4 \\
.5 \\
.6 \\
.7 \\
.8 \\
.9\end{array}$ & $\begin{array}{l}50 \\
00 \\
13 \\
81 \\
40 \\
66 \\
32 \\
11 \\
99 \\
24\end{array}$ & $\begin{array}{l}.050 \\
.100 \\
.213 \\
.381 \\
.440 \\
.566 \\
.637 \\
.711 \\
.899 \\
.924\end{array}$ & $\begin{array}{l}2750 \\
4961 \\
3183 \\
9444 \\
1575 \\
1057 \\
3086 \\
1964 \\
4827 \\
3923\end{array}$ & $\begin{array}{r}4 \\
9 \\
6 \\
10 \\
2 \\
1 \\
5 \\
3 \\
8 \\
7\end{array}$ & $\begin{array}{l}.381 \\
.899 \\
.566 \\
.924 \\
.100 \\
.050 \\
.440 \\
.213 \\
.711 \\
.637\end{array}$ & $\begin{array}{r}-0.30 \\
1.28 \\
0.17 \\
1.43 \\
-1.28 \\
-1.64 \\
-0.15 \\
-0.80 \\
0.56 \\
0.35\end{array}$ & $\begin{array}{l}2.70 \\
4.28 \\
3.17 \\
4.43 \\
1.72 \\
1.36 \\
2.85 \\
2.20 \\
3.56 \\
3.35\end{array}$ \\
\hline
\end{tabular}

Figure 26. A Stratified Sample of Size 10 for $x_{4}$ from a Normal Population with $\mu=3$ and $\sigma 2=1$. 


\begin{tabular}{|c|c|c|c|c|c|}
\hline $\begin{array}{l}\text { (a) } \\
\text { Obs. } \\
\text { No. }\end{array}$ & $\begin{array}{c}\text { (b) } \\
\text { Input } \\
x_{1} \\
\end{array}$ & $\begin{array}{c}\text { (c) } \\
\text { Input } \\
x_{2} \\
\end{array}$ & $\begin{array}{c}\text { (d) } \\
\text { Input } \\
x_{3} \\
\end{array}$ & $\begin{array}{c}\text { (e) } \\
\text { Input } \\
X_{4} \\
\end{array}$ & $\begin{array}{c}\text { (f) } \\
\text { output } \\
Y \\
\end{array}$ \\
\hline $\begin{array}{r}1 \\
2 \\
3 \\
4 \\
5 \\
6 \\
7 \\
8 \\
9 \\
10\end{array}$ & $\begin{array}{r}2.05 \\
0.13 \\
0.67 \\
-0.87 \\
1.74 \\
2.77 \\
0.33 \\
1.21 \\
0.80 \\
1.27\end{array}$ & $\begin{array}{l}2.57 \\
0.19 \\
1.36 \\
1.60 \\
2.07 \\
2.49 \\
3.21 \\
0.83 \\
1.96 \\
3.32\end{array}$ & $\begin{array}{l}1.11 \\
3.81 \\
1.61 \\
2.44 \\
1.88 \\
2.23 \\
1.39 \\
0.26 \\
2.73 \\
3.04\end{array}$ & $\begin{array}{l}2.70 \\
4.28 \\
3.17 \\
4.43 \\
1.72 \\
1.36 \\
2.85 \\
2.20 \\
3.56 \\
3.35\end{array}$ & $\begin{array}{r}5.02 \\
4.16 \\
5.61 \\
6.28 \\
6.02 \\
7.19 \\
10.39 \\
3.00 \\
9.02 \\
12.88\end{array}$ \\
\hline
\end{tabular}

Figure 27. A Latin Hypercube Sample of Size 10 and the Associated Output Y. 


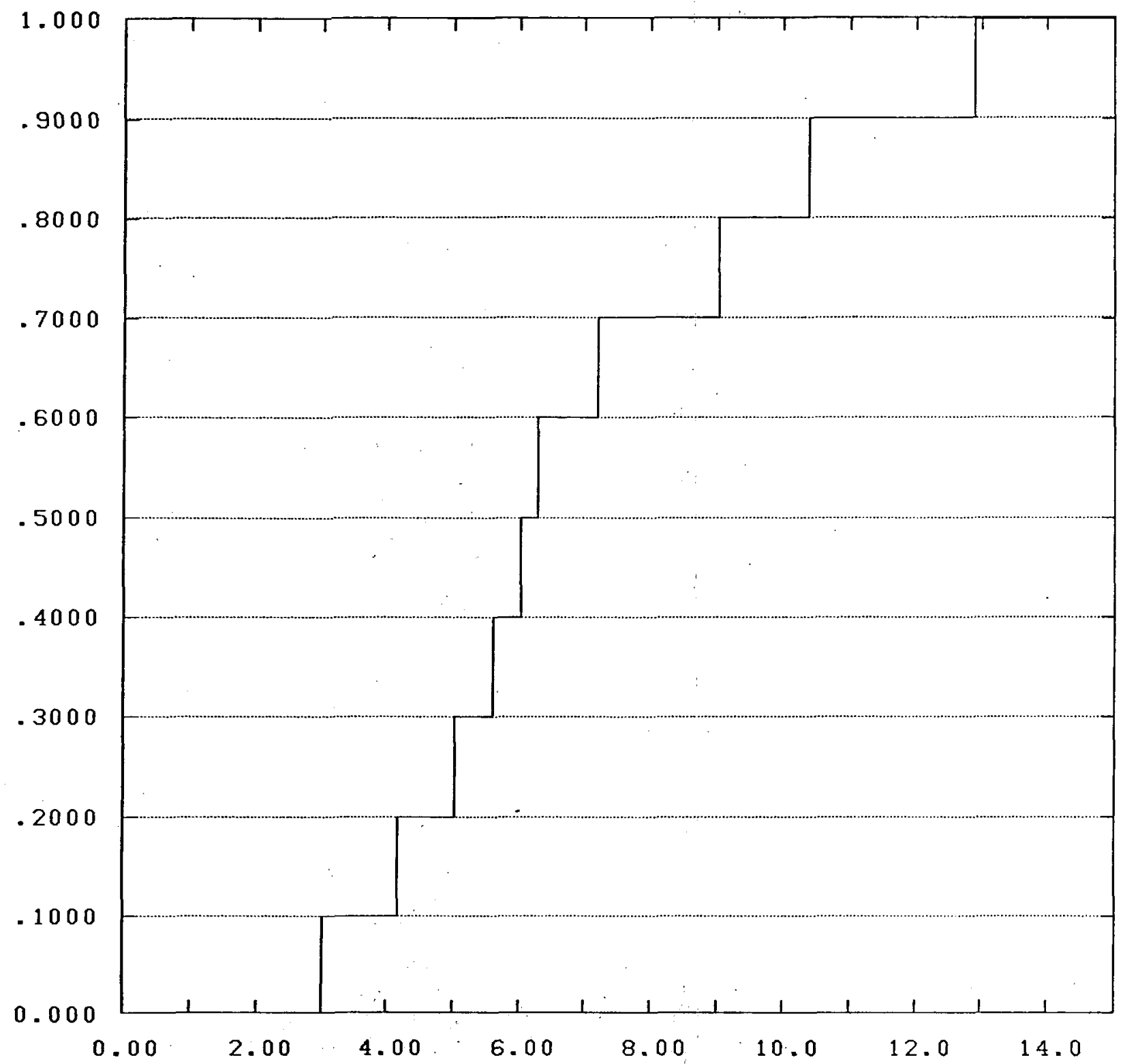

Figure 28. An Empirical Distribution Function for the output in Figure 27. 
Now rearrange the numbers from column $(c)$, putting the smallest number next to rank 1 , the second smallest next to rank 2 , and so on to the largest number which goes next to rank 10 in each Figure. These go in column ( $f)$.

Step 3. Convert the values of $F(x)$ to a stratified sample from $F(x)$. Since $F(x)$ is a normal distribution function, enter the respective row and column of Table 2 as indicated by the value in column ( $f$ ), and record the table entry $\Phi^{-1}$, in column $(g)$. Add the mean of $F(x)$ to column $(g)$ to get the value of $F^{-1}$, which goes in column $(h)$. Column (h) contains the stratified sample from $F(x)$, arranged in random order.

Step 4. Combine the individual stratified samples into a Latin Hypercube Sample. Transcribe the numbers in column (h) of Figure 23 into column (b) of Figure 27 , without changing the relative ordering. In a similar fashion the numbers in column (c) of Figure 27 come from Figure 24, column (d) comes from Figure 25 and column (e) comes from Figure 26. It is important to keep the same relative ordering of the numbers when transcribing them.

Step 5. Obtain the output from the black box model using the Latin Hypercube Sample. The entries in row 1 of Figure 27 are entered into a pre-programmed calculator, or the function

$$
y=x_{1}+x_{2} x_{3}-x_{2} \ln \left|x_{1}\right|+\exp \left(x_{4} / 4\right)
$$

whichever is more convenient, to get the output $Y$ of the black box mode1. Repeat this procedure for each row in Figure 27.

Step 6. Plot an empirical distribution function. Plot the 10 values of $Y$ from Figure 27 onto the horizontal axis of Figure 28 . Draw a step function, starting at zero on the left, and increasing in steps of height $1 / 10$ at each value of $Y$, until the graph reaches a height of 1.0 at the largest value of. $Y$. This is an estimate of the distribution function of the output, obtained using Latin Hypercube Sampling. The average of the 10 values of $Y$ may be used to estimate the population mean; the sample variance, sample median, etc., may be used to estimate the population counterparts.

\section{EXERCISE 6. (Obtaining a Latin Hypercube Sample of size 10)}

Follow the same steps used in the previous example, and obtain a Latin Hypercube Sample of size $N=10$. Use Figures 29-32 to record the steps involved in finding the stratified samples, and put them together in Figure 33 as a Latin Hypercube Sample. Obtain the output values and graph the empirical distribution function in Figure 34. 


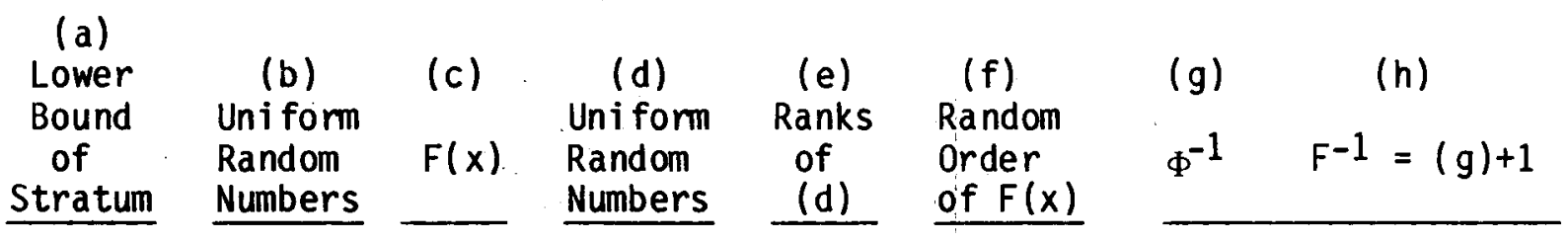

.0

.1

.2

.3

.4

.5

.6

.7

.8

.9

Figure 29. Student Problem: A Stratified Sample of Size 10 for $x_{1}$ from a Normal Population with $\mu=1$ and $\sigma^{2}=1$. 


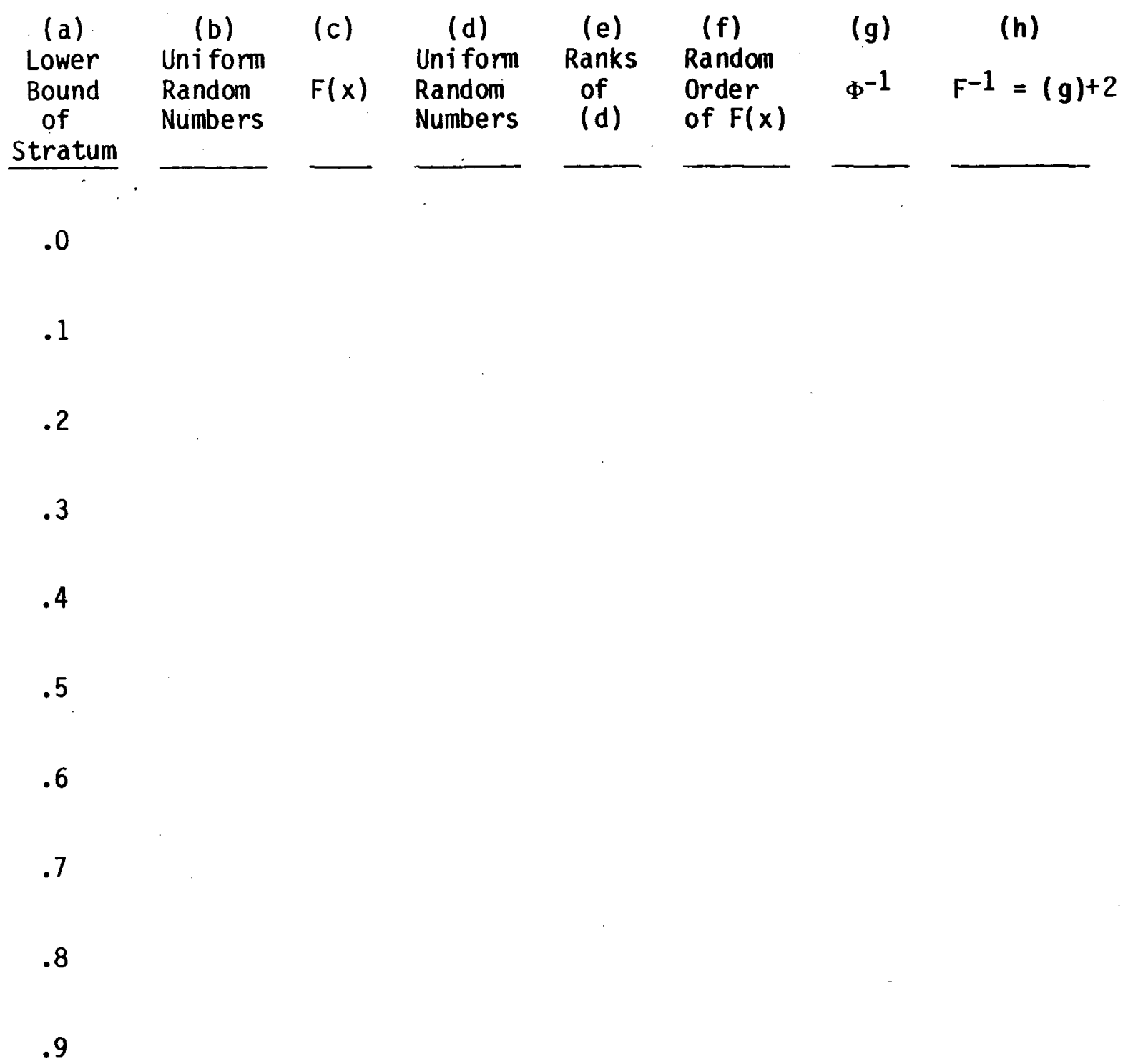

Figure 30. Student Problem: A Stratified Sample of Size 10 for $x_{2}$ from a Normal Population with $\mu=2$ and $\sigma^{2}=1$. 


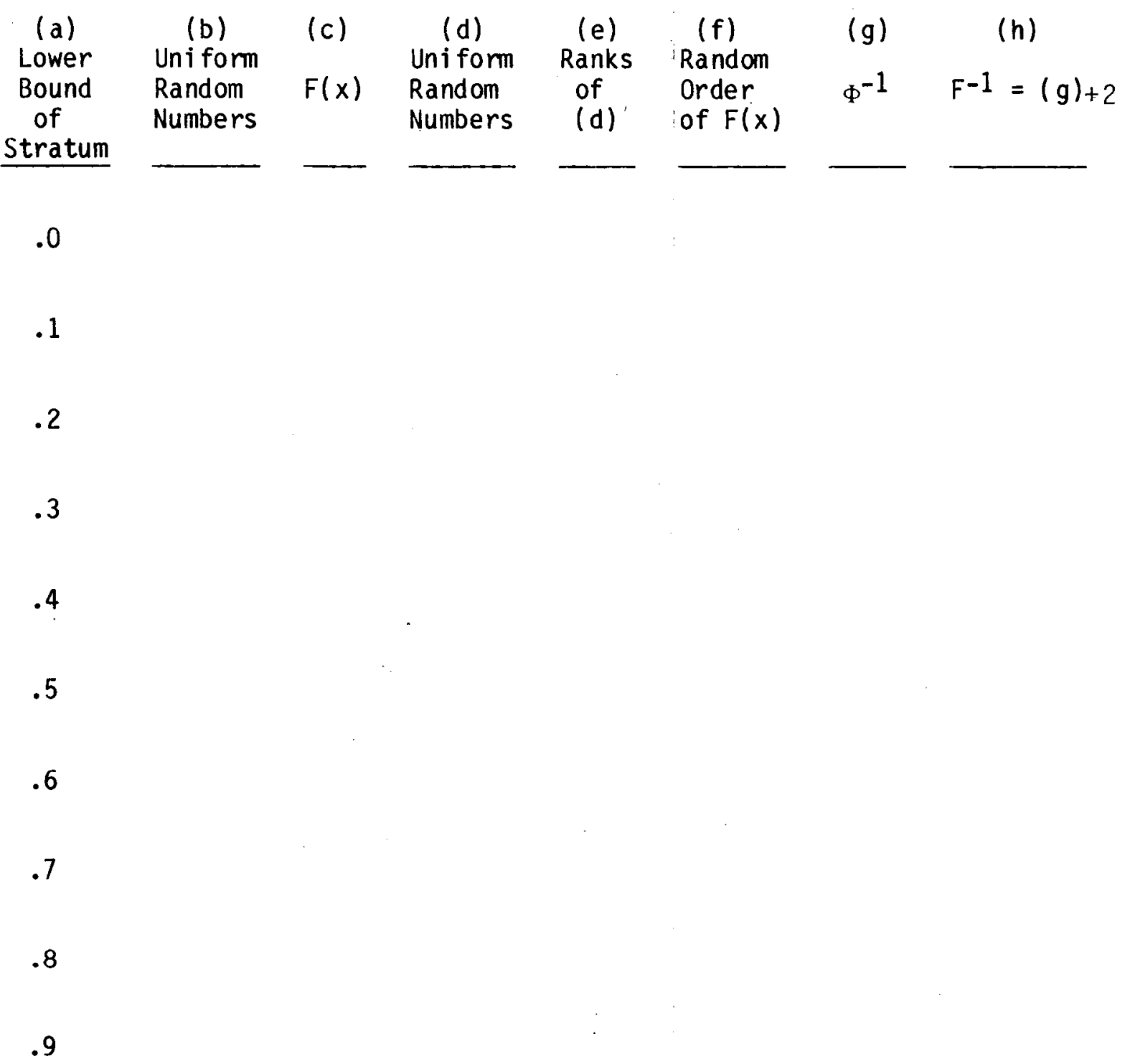

Figure 31. Student Problem: A Stratified Sample of Size 10 for $X_{3}$ from a Normal Population with $\mu i=2$ and $\sigma^{2}=1$. 


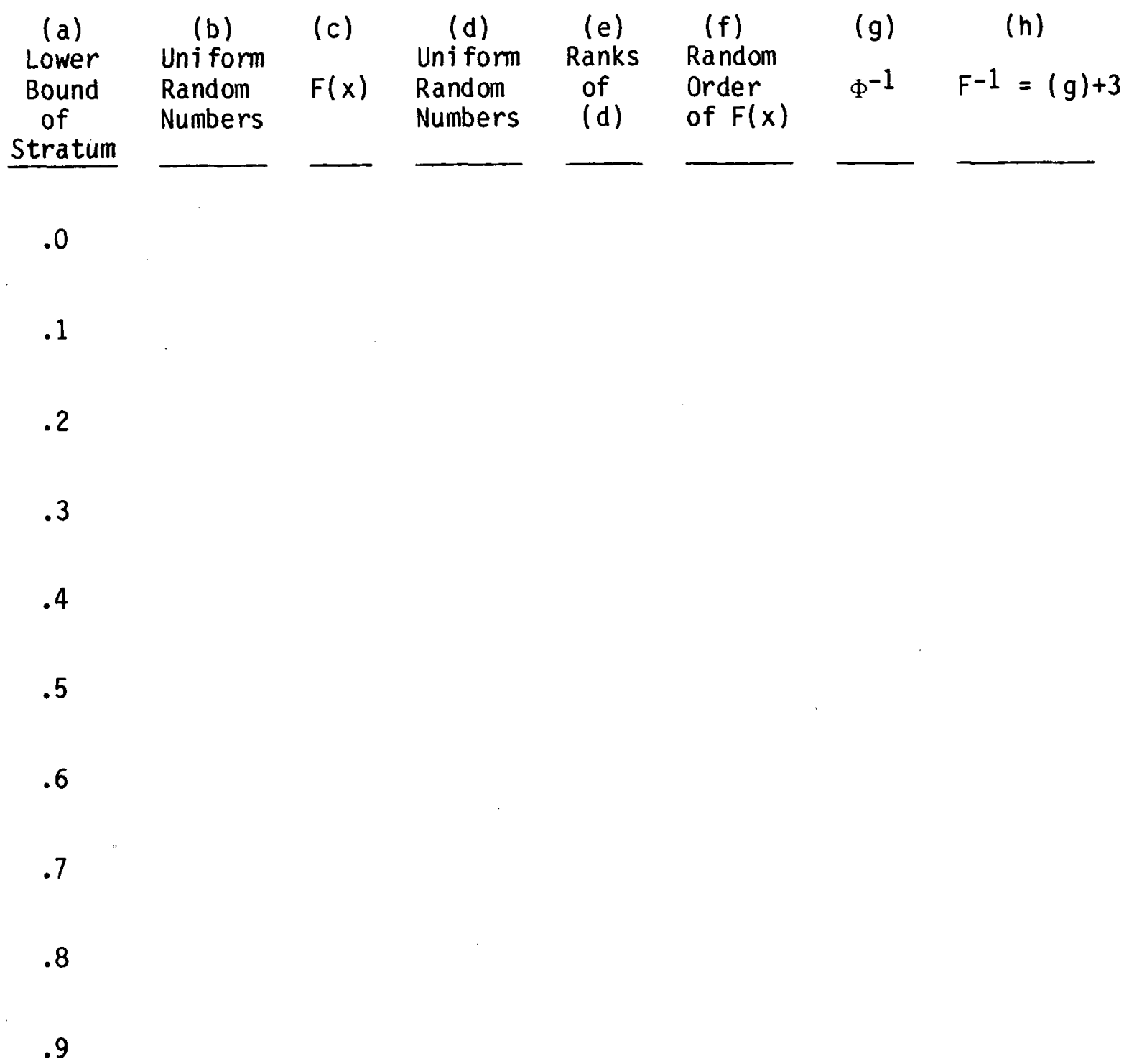

Figure 32. Student Problem: A Stratified Sample of Size 10 for $X_{4}$ from a Normal Population with $\mu=3$ and $\sigma^{2}=1$. 


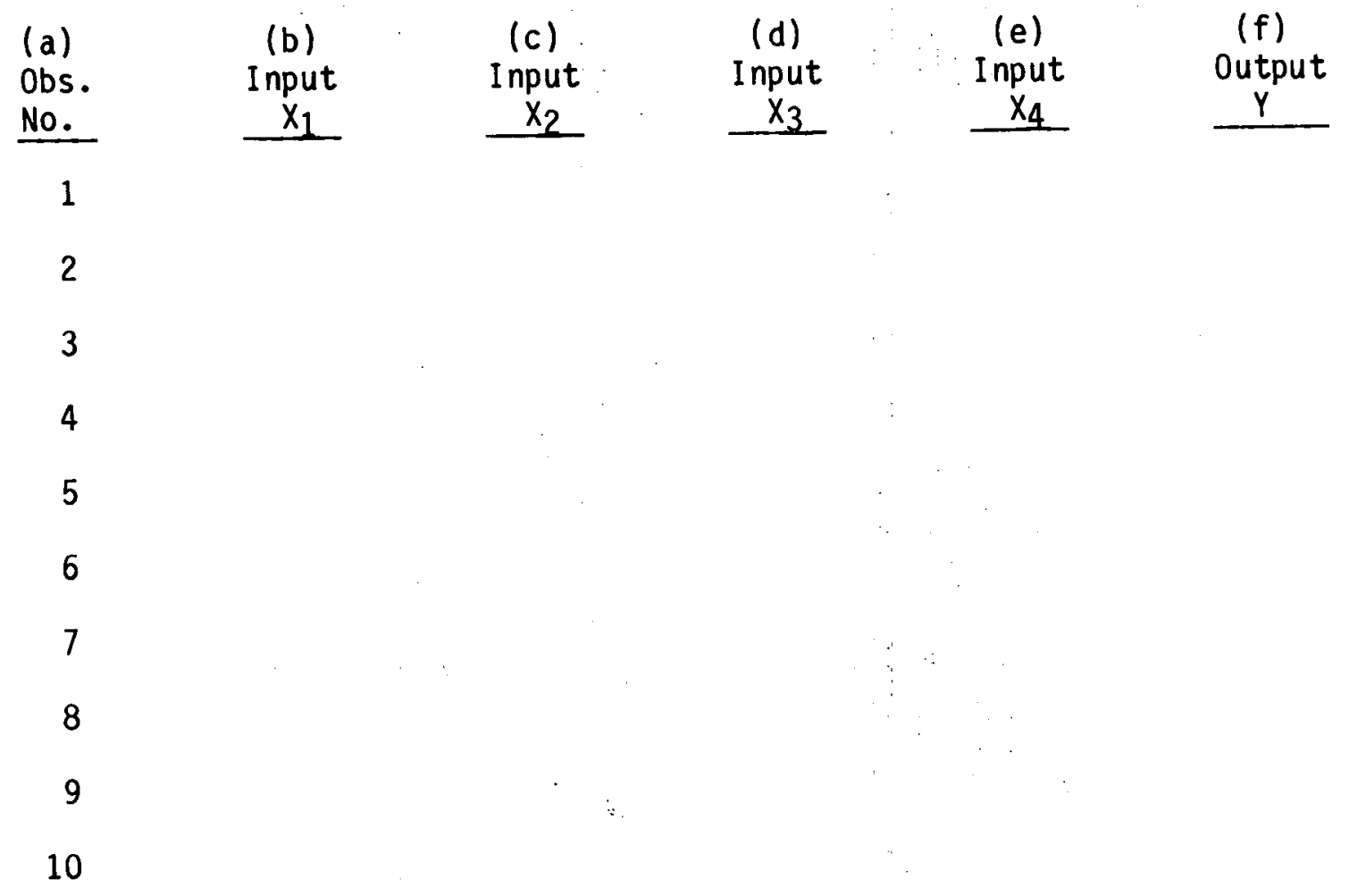

Figure 33. Student Problem: Worksheet for a Latin Hypercube Sample of Size 10 and the Associated Output Y. 


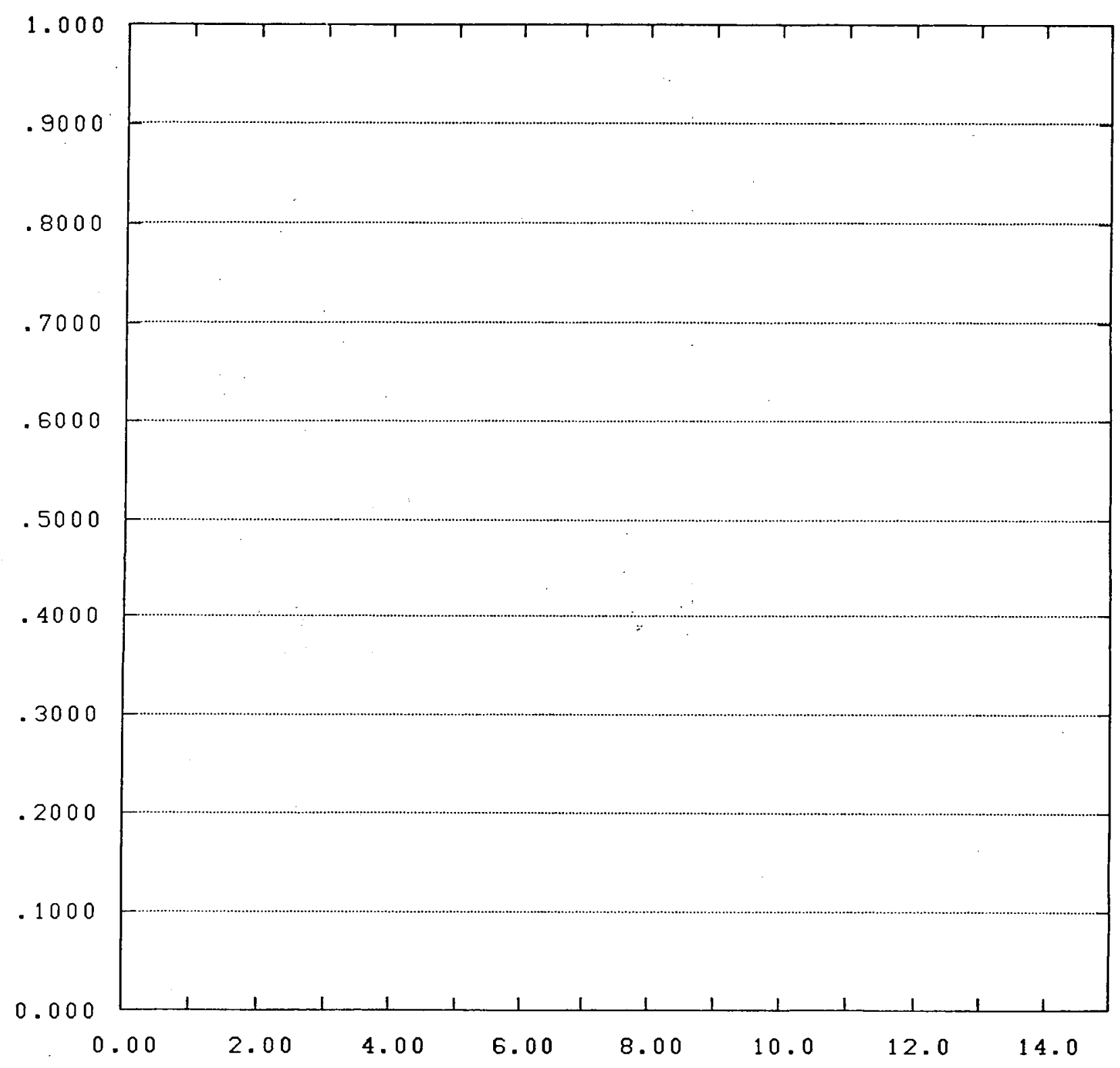

Figure 34. Student Problem: Worksheet for an Empirical Distribution Function for the Output in Figure 33. 
Accuracy Obtained from Using a Latin Hypercube Sample

In order to get some idea of how well a Latin Hypercube Sample of size 10 functions as a basis for estimation, the Latin Hypercube Sampling program was used to obtain five Latin Hypercube Samples of size 10 each. The e.d.f.'s for these five samples appear in Figure $35,(a)-(e)$. The mean of these five graphs, computed in a vertical direction, appears in Figure 36. In the background of Figures 35 and 36 is an estimate of the population distribution function, obtained using a Latin Hypercube Sample of size $N=1000$. This estimate coincides almost perfectly with the estimate in Figures 20 and 21 which was obtained from a Random Sample of size $\mathrm{N}=1000$. This close agreement confirms the fact that both methods of sampling are providing unbiased estimates of the population distribution function.

The mean EDF is plotted again in Figure 37 , along with curves plotted one standard deviation above and bel ow the mean curve. These three curves collectively give some idea of the spread involved using Latin Hypercube Samples of size 10 as estimators of the population distribution function. The standard deviation is computed vertically from the five curves in Figure 35 , and smoothed using a three point moving average.

A Comparison of Latin Hypercube with Random Sampling

A comparison of Figures 36 and 37 with Figures 21 and 22 shows that, in this case, the five Latin Hypercube Samples provide a better composite estimate of the population distribution function than do the five random samples obtained earlier. Because of sampling variability, there is no guarantee that Latin Hypercube samples are always better than random samples, but all of the simulation studies we are aware of indicate a definite tendency in this idirection.

The Replicated Latin Hypercube Sample

When five random samples are pooled together as in Figure 21 the result is another random sample, whose size is equal to the total pooled sample size. However, when five (or any number) Latin Hypercube Samples are pooled together as in Figure 36, the result is called a Replicated Latin Hypercube Sample. Actually the inputs are more correctly called the replicated Latin Hypercube Sample. Replication al lows standard deviations to be computed. These standard deviations should be divided by $\sqrt{r}$, where $r$ is the number of replications, to get an estimate of the standard errorl of the estimate of the mean c.d.f. In the previous example, the standard deviations would be divided by $\sqrt{5}$. The estimate of the true output distribution function obtained from a random sample of size 50, and the standard error of the estimate, is given in Figure 38. This is different than Figure 22 which $i 11$ ustrated the error involved when using a random sample of size 10. The corresponding

T

Standard error refers to the standard deviation of an estimate 


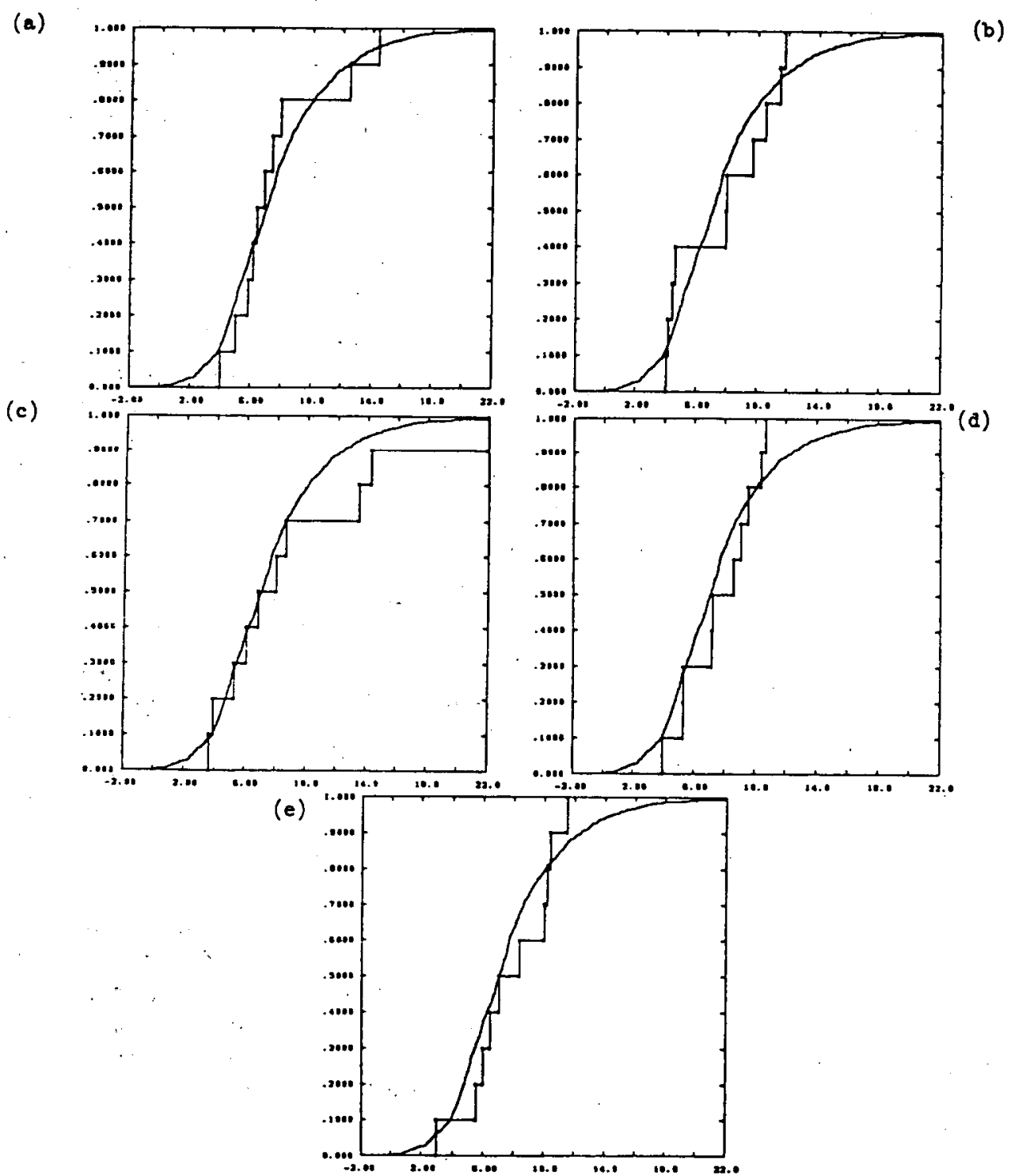

Figure 35. Five EDF's Obtained from Latin Hypercube Samples of Size 10 Each, and an Estimate of the Population Distribution Function 


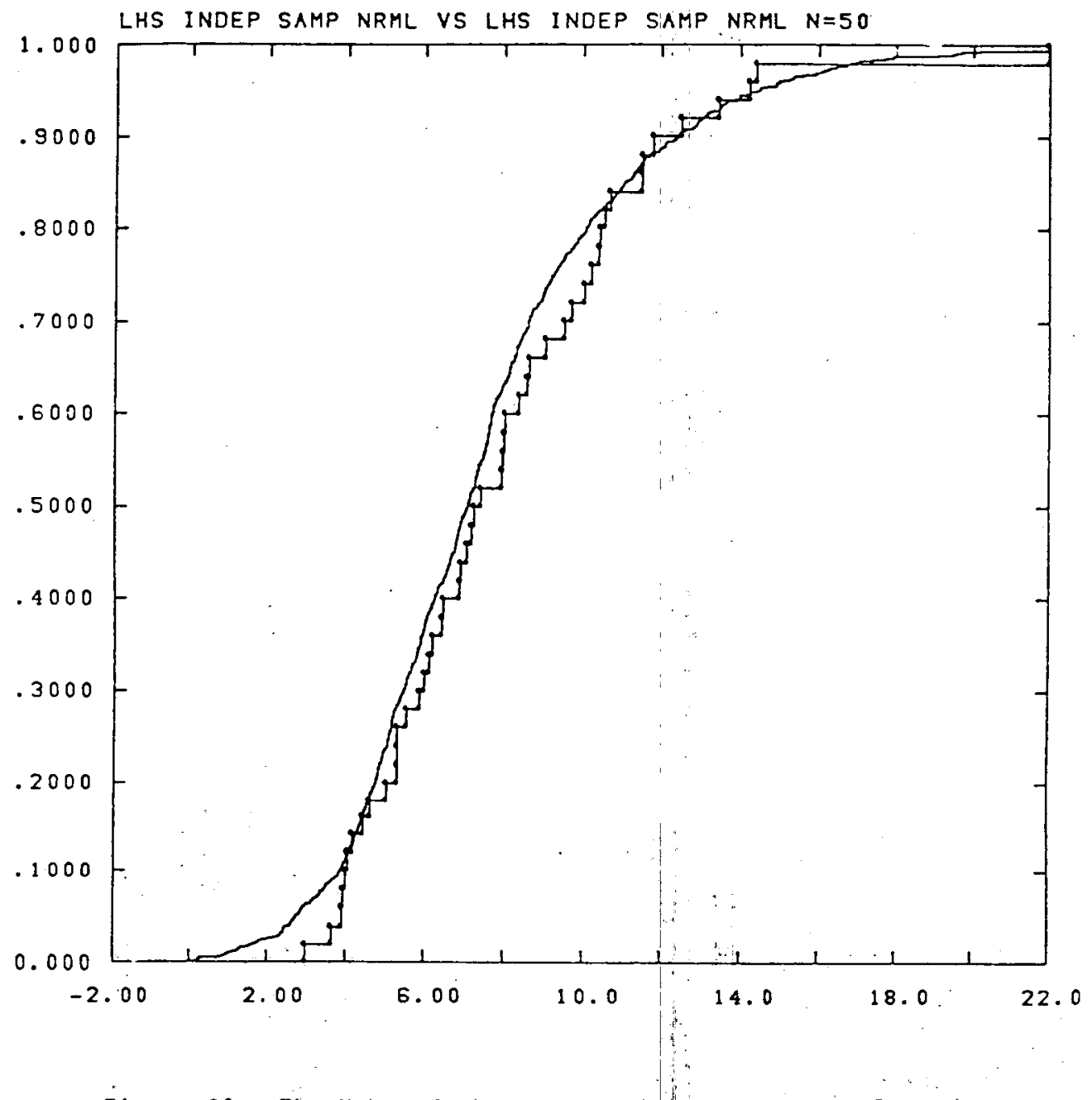

Figure 36. The Mean of the Five EDF's from Figure 35 , and an Estimate of the Populationldistribution Function 


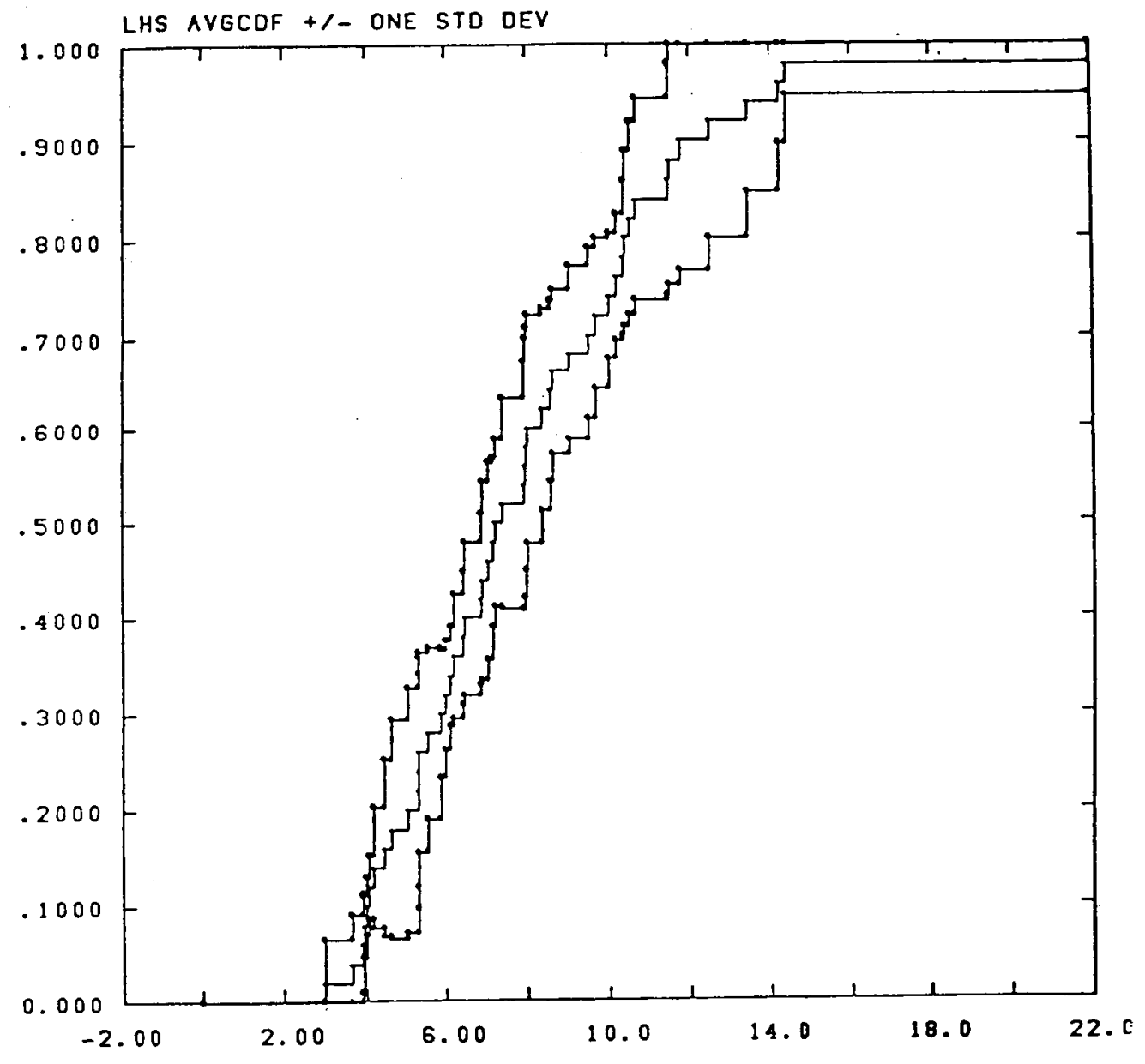

Figure 37. The Mean of the Five EDF's from Figure 35, and One Standard Deviation Bounds 


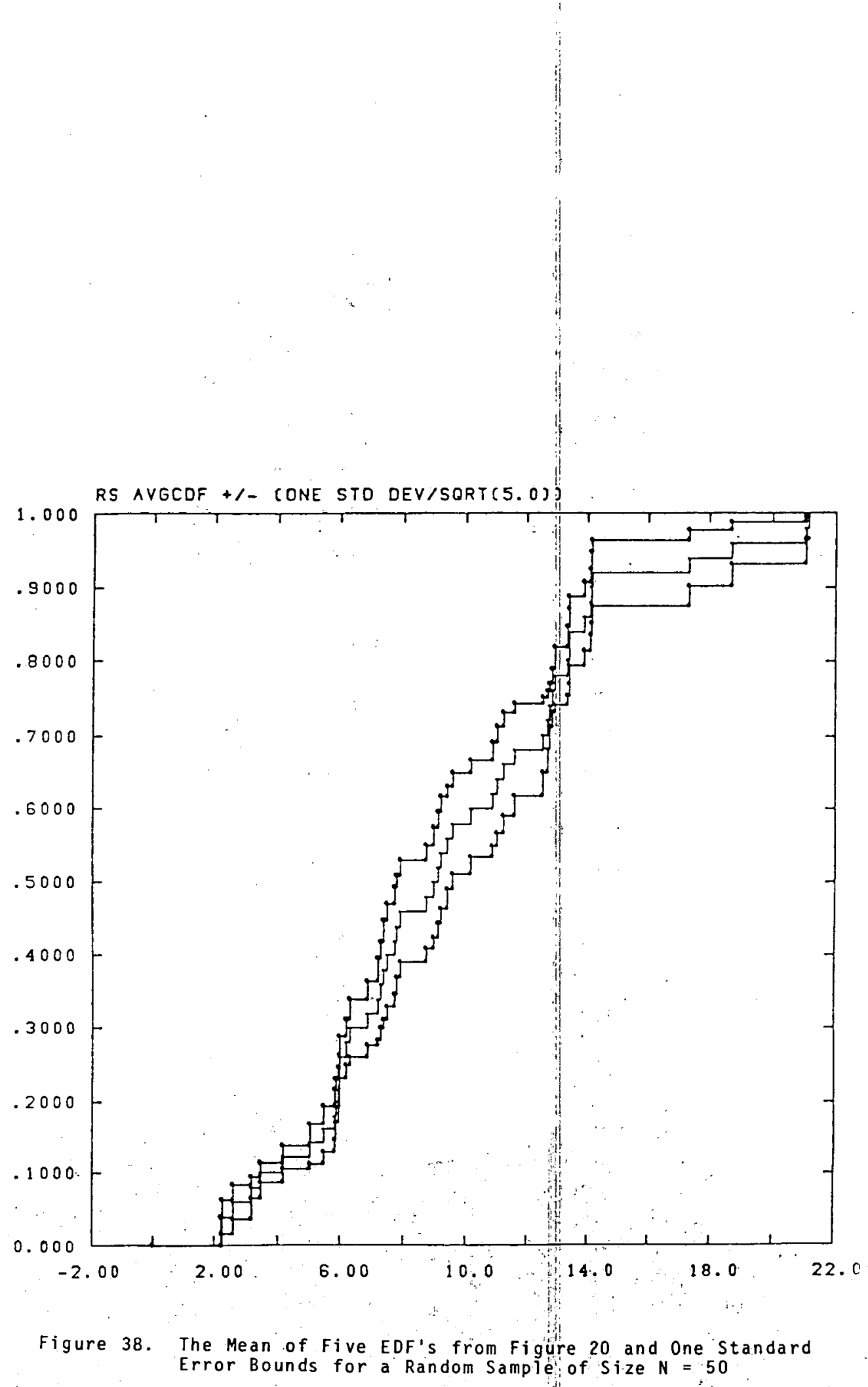


figure for a Replicated Latin Hypercube Sample (5 samples of 10 each) is given in Figure 39, showing the estimated distribution function and the one standard error bounds. This may be contrasted with Figure 37 which illustrated the standard error involved when using a single Latin Hypercube Sample of size 10.

\section{Estimating Other Population Parameters}

Other population quantities are estimated in the usual way from the sample outputs. For example, the sample mean is used to estimate the population mean. Each of the five random samples provides a sample mean, as does each of the five Latin Hypercube samples. These are lis ted below.

True population mean $\mu=7.585$

\begin{tabular}{|c|c|c|c|}
\hline \multicolumn{2}{|c|}{$\begin{array}{c}\text { Random Sample } \\
\text { Estimates }\end{array}$} & \multicolumn{2}{|c|}{$\begin{array}{c}\text { Latin Hypercube Sa } \\
\text { Estimates }\end{array}$} \\
\hline $\begin{array}{r}1 . \\
2 . \\
3 . \\
4 \\
5 . \\
\text { ave. }\end{array}$ & $\begin{array}{r}8.504 \\
9.736 \\
10.778 \\
8.825 \\
10.029 \\
9.575\end{array}$ & $\begin{array}{r}1 . \\
2 . \\
3 . \\
4 . \\
5 . \\
\text { ave. }\end{array}$ & $\begin{array}{l}7.672 \\
7.682 \\
9.266 \\
7.735 \\
7.867 \\
8.044\end{array}$ \\
\hline
\end{tabular}

Latin Hypercube Samples appear to provide better estimates of most, if not all, population parameters when compared with Random Samples. However, this observation is based only on empirical evidence, not theoretical proof, and may not be true in particular cases.

\section{Changes in the Input Distributions}

If the input distributions are changed, the output distribution will be changed also. Just how much the output distribution function will change depends on the degree of change in the input distributions and the strength of the association between the output and each input variable. For purposes of illustration, the input distributions in the example depicted in Figure 2 were changed from normal to uniform for each of the four variables. The range of each variable remained the same. Now the samples of observations from each distribution will not tend to be in the center of the range, as with a normal distribution, but will tend to be spread evenly from one end of the range to the other. This increased emphasis on values in the tails of the range can be expected to alter the output distribution somewhat, but the degree of change is difficult to predict. 


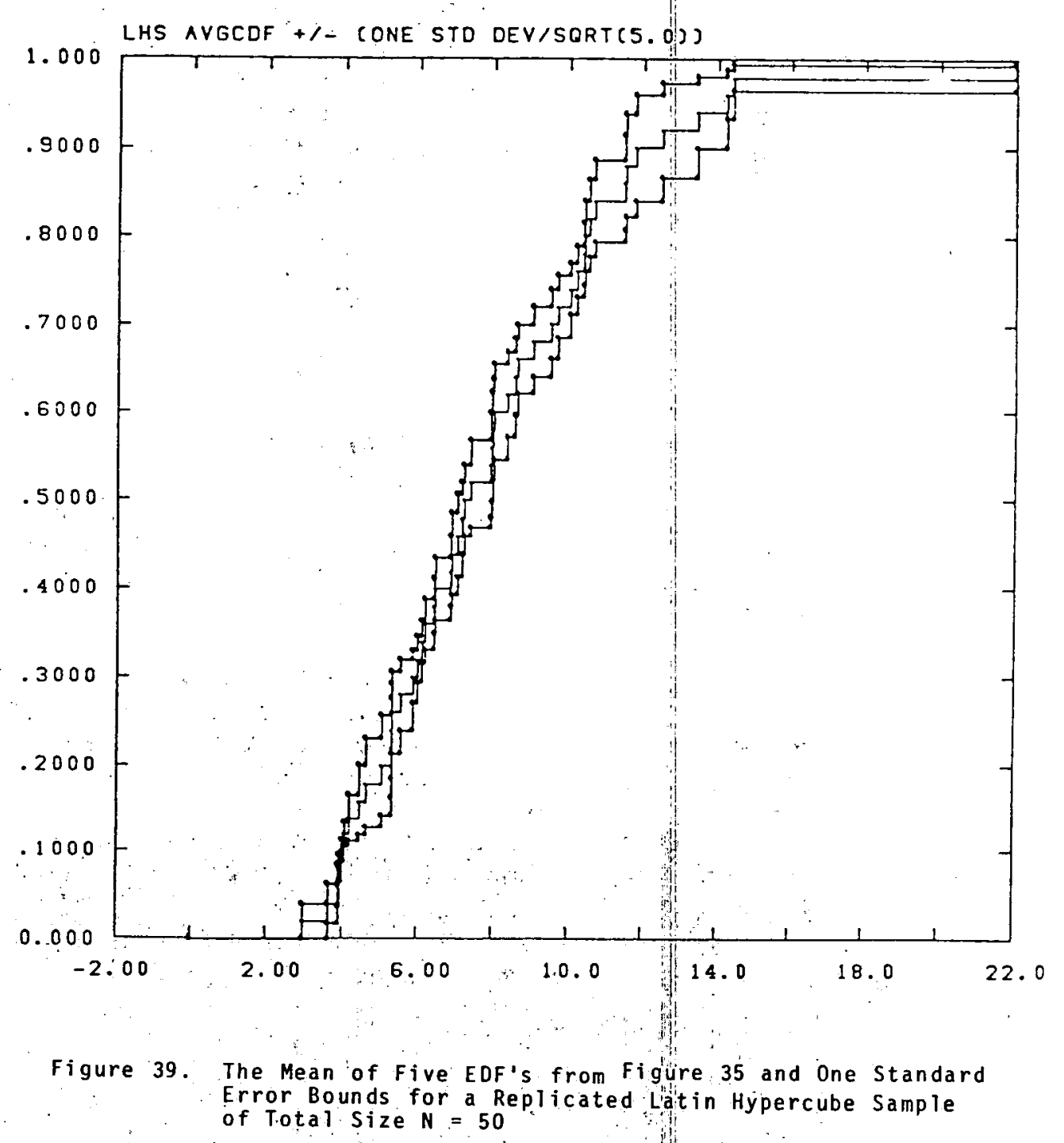




\section{Illustrating the Effect of a Change in Input Distributions}

To see how much change is induced by changing the four input distributions from normal to uniform, a Latin Hypercube Sample of size $N=1000$ was obtained using the Latin Hypercube Sampling Program. The steps involved in finding such a sample are similar to those described earlier. That is, in Figures 23-26, columns (a) through ( $f$ ) would remain unchanged, but to convert from $F(x)$ to $F^{-1}$, as given in column (h), the inverse function for a uniform distribution would be used instead of the inverse function for a normal distribution function. The result for a sample of size 1000 is given in Figure 40 (dark line) and contrasted with the previous case involving normal distributions ( 1 ight line). The large sample size enables these graphs to be treated as if they were the true output distribution functions. The change in the distribution is considerable, which illustrates the importance of being as accurate as possible in specifying the input distribution functions.

\section{The Actual Correlation on the Input Values}

Recall that in drawing a multivariate random sample, the process depended on numbers from a random number generator or, in this case, Table 1. Since the numbers drawn in this way are supposed to be independent of one another, any correlation induced should be spurious correlation due simply to usual random fluctuation one might expect to encounter in random samples.

The same is true for the Latin Hypercube Samples, which were dependent on random numbers for the pairing of values of $x_{1}$ with $x_{2}$ for instance. Since the values of $x_{1}$ and $x_{2}$ were permuted at random, any correlation between $x_{1}$ and $x_{2}$ should be spurious correlation. To see how much correlation actually exists between these randomly permuted values, the actual correlation coefficient was computed on the values of $x_{1}$ and $x_{2}$ given in columns $(b)$ and $(c)$ of Figure 27 . That correlation is $r_{12}=.3595$ which is much less than the $5 \%$ critical value .632 , so a correlation this large can easily be due to chance fluctuations. The entire correlation matrix for the columns in Figure 26 is given in Figure 41. 


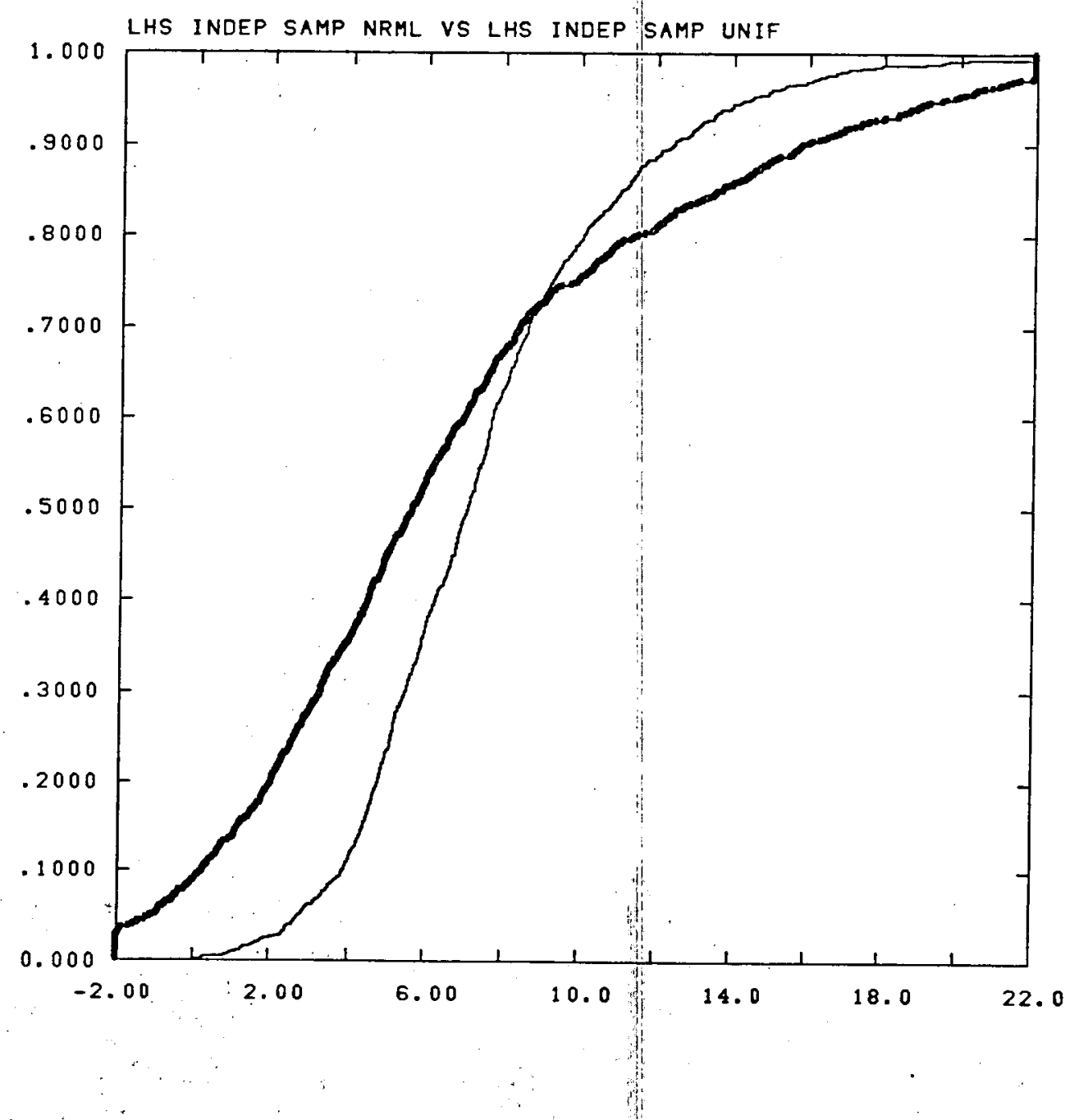

Figure 40. The Output Distribution Function when the Inputs are Uniformly Distributed, Contrasted With the Case of Normal Input Variables. 


$\begin{array}{cccc} & x_{1} & x_{2} & x_{3} \\ x_{2} & .3595 & & \\ x_{3} & -.2822 & -.1024 & \\ x_{4} & -.8535 & -.3170 & .5565\end{array}$

Figure 41. The Correlation Matrix for the Latin Hypercube Sample in Figure 27.

Note that five of the six correlation coefficients are nonsignificant at the $5 \%$ level. The correlation between $X_{1}$ and $X_{4}$ is -.8535 , which exceeds in absolute value the critical value, but this is merely a chance occurrence, since the sampling method does not induce any systematic correlation in the values. Also, the critical value .632 applies to random samples; the exact critical value for Latin Hypercube Samples is unknown.

The Rank Correlation on the Input Values

Since the exact behavior of the correlation coefficient from Latin Hypercube Samples is not known, and since its behavior even with random sampling is unknown, if the input distributions are not normal, it makes more sense to work with the rank correlation coefficient, known as Spearman's rho, and which is simply $r$ computed on the ranks of the data. The behavior of the rank correlation coefficient is the same for Latin Hypercube Samples as it is for random samples, and is the same for all types of input distributions.

The ranks of the Latin Hypercube Sample of Figure 27 are given in column (e) of Figures 23-26, and are reproduced in Figure 42 for the reader's convenience. The rank correlation matrix for these ranks is given in Figure 43 . The $5 \%$ critical value for the rank correlation coefficient is .6364 . Note that, as before, the only correlation that exceeds this value in absolute value is the rank correlation between $X_{1}$ and $X_{4}$. Some of the other correlations tend to be large also, such as $\rho_{34}=.6121$ between $X_{3}$ and $x_{4}$.

Some Undesirable Effects of Spurious Correlation

These large correlation coefficients that occur by chance after a random permutation of the input variables are annoying for several reasons. For one, the independence assumption between input variables implies that the population correlations equal zero. Since the sample correlations act as estimates of the population values, it would be 

desirable for the sample correlations to be close to zero if possible. In this way, the sample input values would be more "typical" of the population, and this should be reflected in more confidence in the output being more typical of the population output.

A second reason for wanting smaller correlation is that large correlation tends to introduce an effect known as multi-colinearity, which is acceptable if the variables are actually related, but which may be undesireable if the variables are actually independent. For these reasons, a method for reducing sample correlations of input values is desirable. Such a feature is built into the Latin Hpercube Sampling Program.

\section{Reducing the Spurious Correlation}

The Latin Hypercube Sampling program does not obtain a random pairing of the input vectors in either the random sampling option or the Latin Hypercube option. Rather, it pairs the variables so they will have correlation coefficients closer to the population correlation coefficients, in order to reduce the undesirable effects associated with spurious correlation. For the first random sample of size 10, whose e.d.f. is given in Figure 20(a), the input variables were arranged so that their ranks matched the ranks given in Figure 44 . That is, instead of being satisified with a random ordering such as in column (e) of Figures 14-17, the values are arranged so that their ordering agrees with the ordering in Figure 44. Then the rank correlations are given in Figure 45. Note that the largest rank correlation in Figure 45 is .2217 , and that four of the six correlations are less than .1. These correlations as a group tend to be closer to the zero population value that is appropriate for independent input variables. Note also that the rank correlations in Figure 45 depend only on the ranks in Figure 44 , and not in any way on the input distributions or the particular input values.

\section{An Illustration of Reducing the Correlation}

The same rank ordering given in Figure 44 was used on both the first random sample and the first Latin Hypercube Sample, whose e.d.f. is given in Figure 35(a). To illustrate how this is accomplished, the example given in Figures 23-27 will be reworked so that the correlation matrix of the input values will be the same as in Figure 45 . This means that the ranks of the input values need to agree with the columns of Figure 44. For variable $X_{1}$ the original ordering and the new ordering are given in Figure 46. The original ordering was given in Figure 23, column (h) and the original ranks were given in the same figure, column (e). The new rank ordering for $X_{1}$ is given in Figure 44 , column (b). Since the new ordering has rank 8 in run number. 1 , the $x_{1}$ value with rank $8, x_{1}=1.74$, is now listed first. All of the values of $x_{1}$ are thus arranged to agree with the new rankings, as illustrated in Figure 46. 


\begin{tabular}{|c|c|c|c|c|}
\hline $\begin{array}{l}\text { (a) } \\
\text { Run }\end{array}$ & (b) & (c) & (d) & (e) \\
\hline Number & $x_{1}$ & $x_{2}$ & $x_{3}$ & $x_{4}$ \\
\hline 1 & 8 & 6 & 1 & 9 \\
\hline 2 & 7 & 7 & 10 & 5 \\
\hline 3 & 2 & 2 & 6 & 6 \\
\hline 4 & 4 & 5 & 3 & 7 \\
\hline 5 & 9 & 10 & 5 & 3 \\
\hline 6 & 6 & 4 & 9 & 1 \\
\hline 7 & 1 & 9 & 2 & 2 \\
\hline 8 & 10 & 3 & 4 & 8 \\
\hline 9 & 5 & 1 & 7 & 4 \\
\hline 10 & 3 & 8 & 8 & 10 \\
\hline
\end{tabular}

Figure 44. The Rank Ordering Induced on the Random Sample of Size $N=10$, Whose Output EDF is Given in Figure $20(a)$.

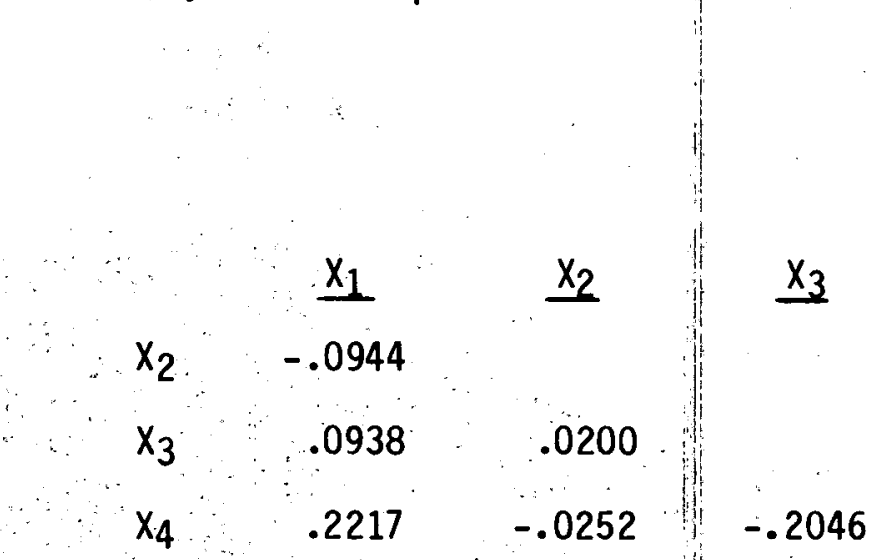

Figure 45. The Rank Correlation Coefficients for the Ranks in Figure 44 . 


\begin{tabular}{ccccc}
$\begin{array}{c}\text { (a) } \\
\text { Run }\end{array}$ & $\begin{array}{c}\text { (b) } \\
\text { Original } \\
\text { Ordering } \\
\text { Fig. 23, } \\
\text { col. (h) }\end{array}$ & $\begin{array}{c}\text { (c) } \\
\text { Original } \\
\text { Ranks } \\
\text { Fig. } 23 \\
\text { col. (e) }\end{array}$ & $\begin{array}{c}\text { (d) } \\
\text { New } \\
\text { Ranks } \\
\text { Fig. }\end{array}$ & $\begin{array}{c}\text { (e) } \\
\text { col. (b) }\end{array}$ \\
\hline 1 & $\begin{array}{c}\text { New } \\
\text { Ordering }\end{array}$ & \\
\hline 2 & 0.05 & 9 & 8 & 1.74 \\
3 & 0.67 & 4 & 7 & 1.27 \\
4 & -0.87 & 1 & 2 & 0.13 \\
5 & 1.74 & 8 & 4 & 0.67 \\
6 & 2.77 & 10 & 9 & 2.05 \\
7 & 0.33 & 3 & 6 & 1.21 \\
8 & 1.21 & 6 & 1 & -0.87 \\
9 & 0.80 & 5 & 10 & 2.77 \\
10 & 1.27 & 7 & 5 & 0.80 \\
& & & 3 & 0.33
\end{tabular}

Figure 46. Changing the Order of the Values of $x_{1}$ to Reduce the Spurious Correlation. 
Similarly the values of $x_{2}$ are arranged in the ordering suggested by the ranks in column (c) of Figure 44. Columns (d) and (e) of Figure 44 define the new orderings of the values of $x_{3}$ and $x_{4}$. When all of the input variables are arranged in the order specified by Figure 44 , their rank correlation matrix will be the same as the one in Figure 45. Of course; the output values $Y$ will not be the same as before since the combinations of input values are now different. However, these new output values are treated the same as any other output values; e.d.f.'s are plotted as they were in Figures 20 and 35 , sample means and sample standard deviations are computed, and so on.".

Simulating Correlated Input Variables

The same principle that is used to make the sample correlations close to zero is used by the Latin Hypercubel Sampling program to make the sample correlation close to any target correlation. That is, first the pairings of ranks are found that result in a desired sample rank correlation coefficient, and then the sample values are arranged in the order suggested by the ranks. These sample correlation coefficients will not equal exactly their target correlations, just as the sample correlations in the previous example did not equal exactly zero, but they will usually be fairly close. The Latin Hypercube Sampling program furnishes a matrix of ranks to use for the ordering of the sample values, and al so furnishes the sample correlation matrix associated with those ranks. If the sample correlation matrix is unsatisfactory for any reason, the program can be used again and again to furnish new rank orderings until a rank ordering with a satisfactory sample rank correlation matrix is found. (See Iman and Conover, 1980).

\section{Illustration of Correlating Input Variables}

Suppose the target correlation matrix is given by Figure 47 . These values are supplied to the Latin Hypercube Sampling program, and a matrix of ranks that may be used is supplied: One such matrix is given in Figure 48. Note that any sample of size 10 for each of four input values may be arranged in the order suggested by Figure 48 . It does not matter if a random sample is used, or ifila Latin Hypercube Sample is used, or what types of input distributions are used. When the sample values have the ordering of Figure 48, they will have the sample rank correlation coefficients given in Figure 49.

The sample values from the Latin Hypercube sample given in Figure 27 are rearranged in the order suggested by Figure 48 . First the $x_{1}$ values are rearranged so that their rank ordering is changed from the former order, 9, 2, 4, 1,8,10,3,6,5,7, as given in column (e) of Figure 23, to the new order $1,9,8,3,7,4,2,6,5,10$, as given in column (b) of Figure 48:. This is the same type of procedure that was illustrated in Figure 46; only the new ordering is different, since now the objective is to achieve correlations close to zero, as was formerly the case. 


$\begin{array}{llll} & \underline{x_{1}} & \underline{x_{2}} & \underline{x_{3}} \\ x_{2} & .8 & & \\ x_{3} & .3 & .4 & \\ x_{4} & .6 & .9 & .7\end{array}$

Figure 47. A Target Correlation Matrix for Four Input Variables.

\begin{tabular}{|c|c|c|c|c|}
\hline $\begin{array}{l}\text { (a) } \\
\text { Run }\end{array}$ & $\begin{array}{l}\text { (b) } \\
x_{1}\end{array}$ & $\begin{array}{r}(c) \\
x_{2}\end{array}$ & $\begin{array}{r}(d) \\
x_{3}\end{array}$ & $\begin{array}{r}(e) \\
X_{4}\end{array}$ \\
\hline Number & $\therefore$ & 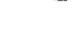 & $\overline{-}$ & 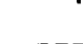 \\
\hline 1 & 1 & 2 & 2 & 3 \\
\hline 2 & 9 & 10 & 9 & 10 \\
\hline 3 & 8 & 8 & 8 & 8 \\
\hline 4 & 3 & 3 & 10 & 6 \\
\hline 5 & 7 & 9 & 7 & 9 \\
\hline 6 & 4 & 7 & 3 & 7 \\
\hline 7 & 2 & 1 & 6 & 2 \\
\hline 8 & 6 & 5 & 4 & \\
\hline 9 & 5 & 4 & 1 & 1 \\
\hline 10 & 10 & 6 & 5 & a \\
\hline
\end{tabular}

Figure 48. Rank Orderings to Achieve Correlations Close to Those in Figure 47.

$\begin{array}{llll} & \underline{x_{1}} & \underline{x_{2}} & \underline{x_{3}} \\ x_{2} & .7939 & & \\ x_{3} & .3212 & .3576 & \\ x_{4} & .5152 & .8545 & .6606\end{array}$

Figure 49. Sample Rank Correlation Matrix for Ranks in Figure 48. 
After rearranging the order of the sample values for $x_{1}$ through $x_{4}$ into the order suggested by the ranks given in:|Figure 48 , the new arrangements are given in Figure 50. The values given in columns (b) through (e) of Figure 50 are obtained from column (h) of Figures 23 through 26 respectively. The ranks in Figure 50 are the ranks in Figure 48 , so the sample rank correlation matrix of the data given in Figure 50 is given by Figure 49 .

Note that even though this example happened to use a Latin Hypercube Sample, a random sample could have been used just as well. All that is required is that the values have the same ordering as given in Figure 48, and the sample rank correlation matrix will be the one in Figure 49.

A New Output Distribution Function

For the sake of illustration, the new combinations of input values shown in Figure 50 were entered into the black box model. The output values are 1 isted in column ( $f$ ) of Figure 50. These are different output values than those given in Figure 27 , because the input values occur in different combinations than before. The e.d.f. is therefore different than before. This is as it should be because the population distribution function being estimated is different than it was before. That is, the true output distribution function depends on what correlations the input variables have, as well as what the input distributions are. The difference in the output distributions due to the correlations structure, Figure 47 , being assumed rather than as suming independence of the input variables is shown by the difference in the two curves in Figure 51. The darker curve in. Figure 51 is the true output distribution when the inputs are correlated, and the lighter curve is the one which results from independent inputs. Because of this difference, it is important for the input variables to simulate the population correlation matrix, through the use of some device such as the one built into the Latin Hypercube Sampling program.

How Many Runs are Needed?

One of the main advantages of the Latin hypercube sampling procedure is that the number of runs can be very small, regardless of the number of variables involved. In fact, there is no lower limit to the number of runs (input vectors) if the user is willinglto sacrifice some of the statistical analyses that are available with larger numbers of runs.

To be able to use the correlation reduction techniques, the number of runs needs to exceed the number of variables. This procedure is more. stable if the number of runs exceeds the number of variables by approximately 25\%. This is also a minimum requirement for stability in the regression procedures and partial correlation coefficients described in the next part of this tutarial. Of course, more runs than this results in even more stability, and for best results, the number of runs should be about two or three times the number of variables involved if time and money permit. 


\begin{tabular}{|c|c|c|c|c|c|c|c|c|c|}
\hline $\begin{array}{c}\text { (a) } \\
\text { Run } \\
\text { Number } \\
\end{array}$ & $\begin{array}{c}\text { Input } \\
x_{1} \\
\end{array}$ & $\begin{array}{l}\text { (b) } \\
\text { (rank) }\end{array}$ & $\begin{array}{c}\text { Input } \\
x_{2} \\
\end{array}$ & (rank) & $\begin{array}{r}\text { Input } \\
x_{3} \\
\end{array}$ & rank & $\begin{array}{r}\text { I nput } \\
x_{4} \\
\end{array}$ & (rank) & $\begin{array}{c}\text { (f) } \\
\text { output } \\
Y \\
\end{array}$ \\
\hline 1 & -0.87 & (1) & 0.83 & (2) & 1.11 & (2) & 2.20 & (3) & 1.90 \\
\hline 2 & 2.05 & (9) & 3.32 & (10) & 3.04 & (9) & 4.43 & $(10)$ & 12.79 \\
\hline 3 & 1.74 & (8) & 2.57 & $(8)$ & 2.73 & (8) & 3.56 & (8) & 9.77 \\
\hline 4 & 0.33 & (3) & 1.36 & (3) & 3.81 & (10) & 3.17 & (6) & 9.23 \\
\hline 5 & 1.27 & (7) & 3.21 & (9) & 2.44 & (7) & 4.28 & (9) & 11.25 \\
\hline 6 & 0.67 & (4) & 2.49 & (7) & 1.39 & (3) & 3.35 & (7) & 7.44 \\
\hline 7 & 0.13 & (2) & 0.19 & (1) & 2.23 & (6) & 1.72 & $(2)$ & 2.48 \\
\hline 8 & 1.21 & (6) & 1.96 & (5) & 1.61 & (4) & 2.85 & (5) & 6.03 \\
\hline 9 & 0.80 & (5) & 1.60 & (4) & 0.26 & (1) & 1.36 & (1.) & 2.98 \\
\hline 10 . & 2.77 & $(10)$ & 2.07 & $(6)$ & 1.88 & (5) & 2.70 & (4) & 6.52 \\
\hline
\end{tabular}

Figure 50. New Input Values with Rank Correlation Matrix Given by Figure 49 . 


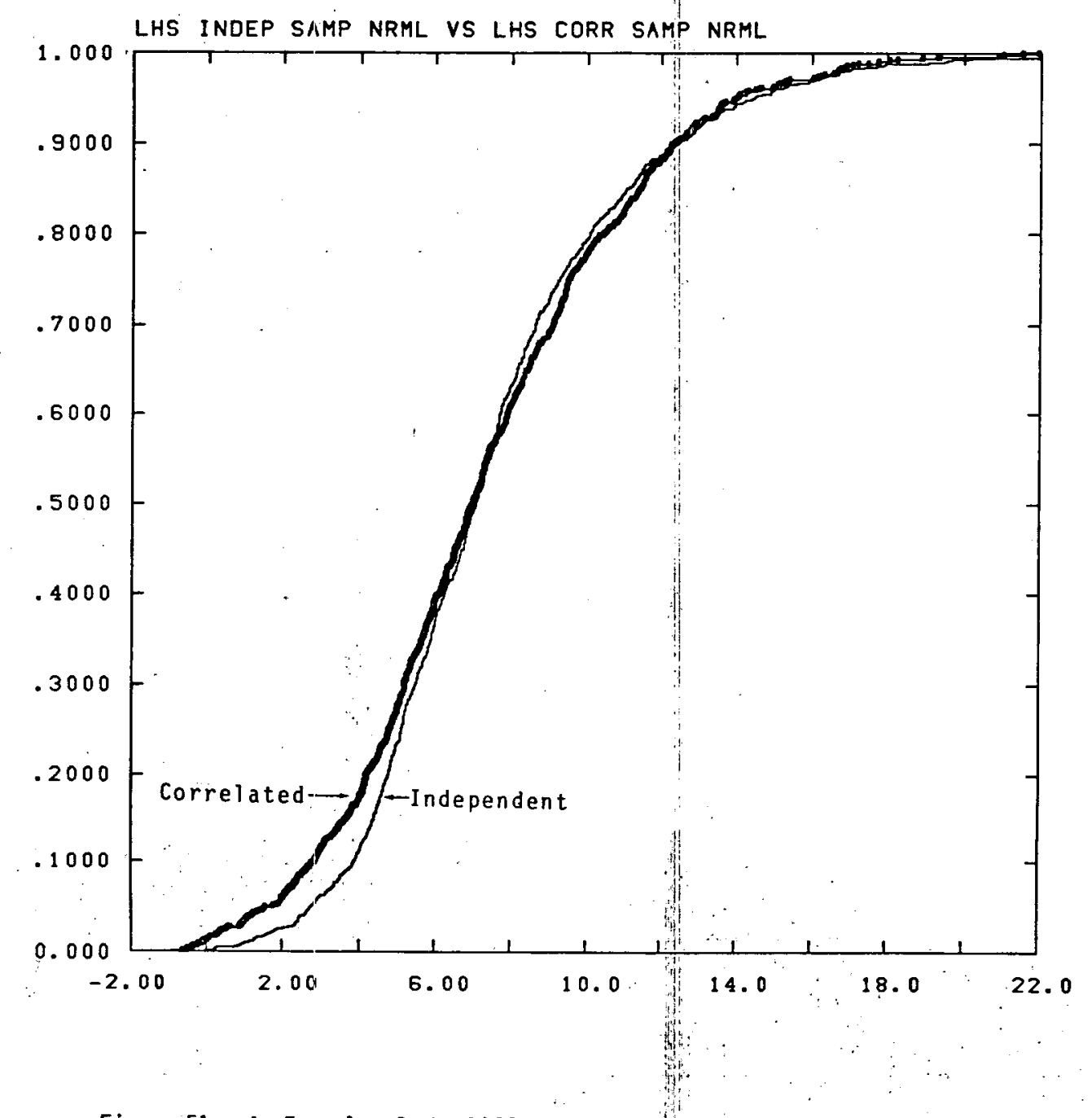

Figure 51. An Example of the Differences in Output Distributions Obtained, Assuming Input Variable Independence and Assuming a Correlation Between Input Variables. 


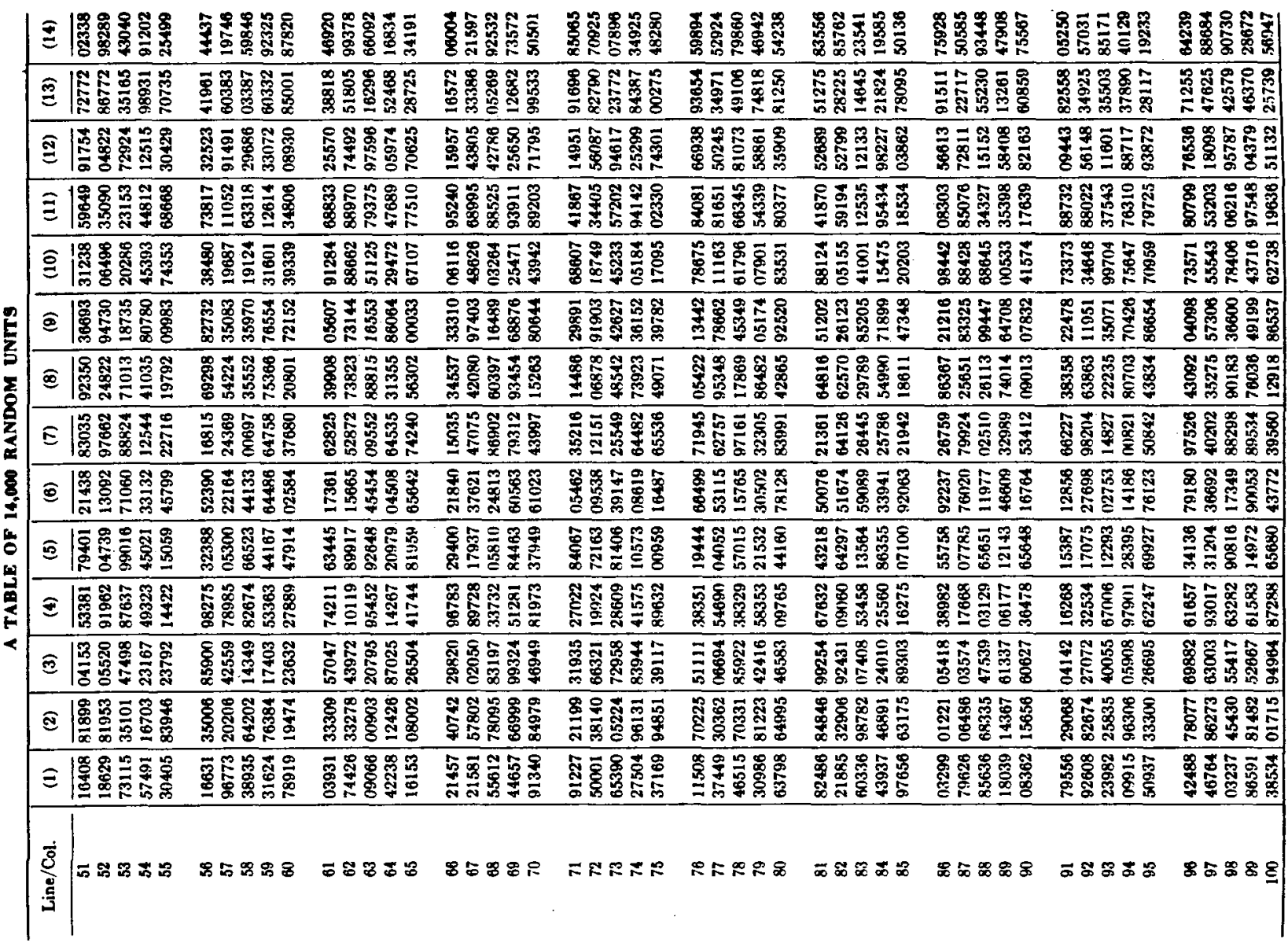

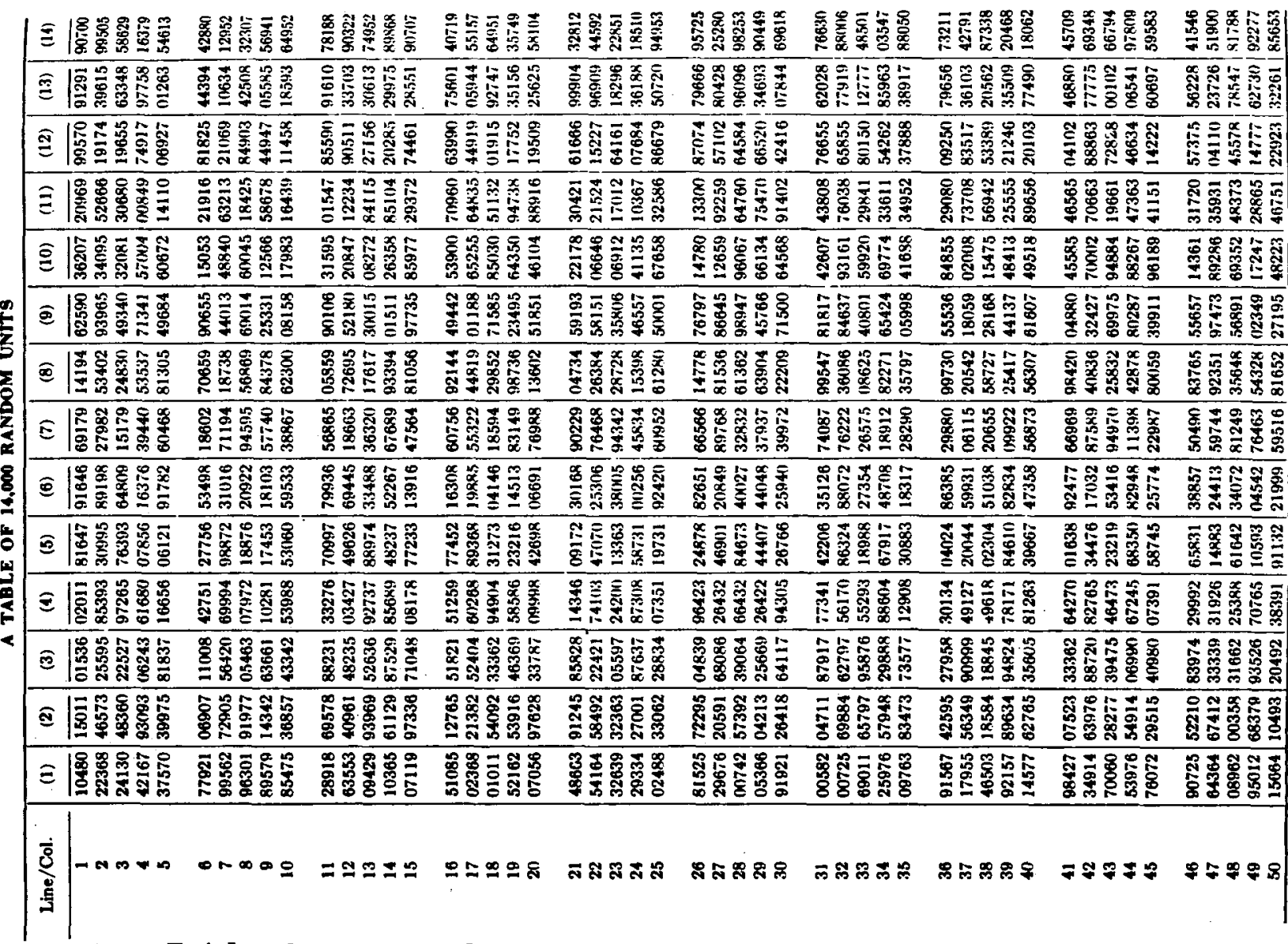

Table 7 A Tabte of Uniform Random Numbers $-62-$ 


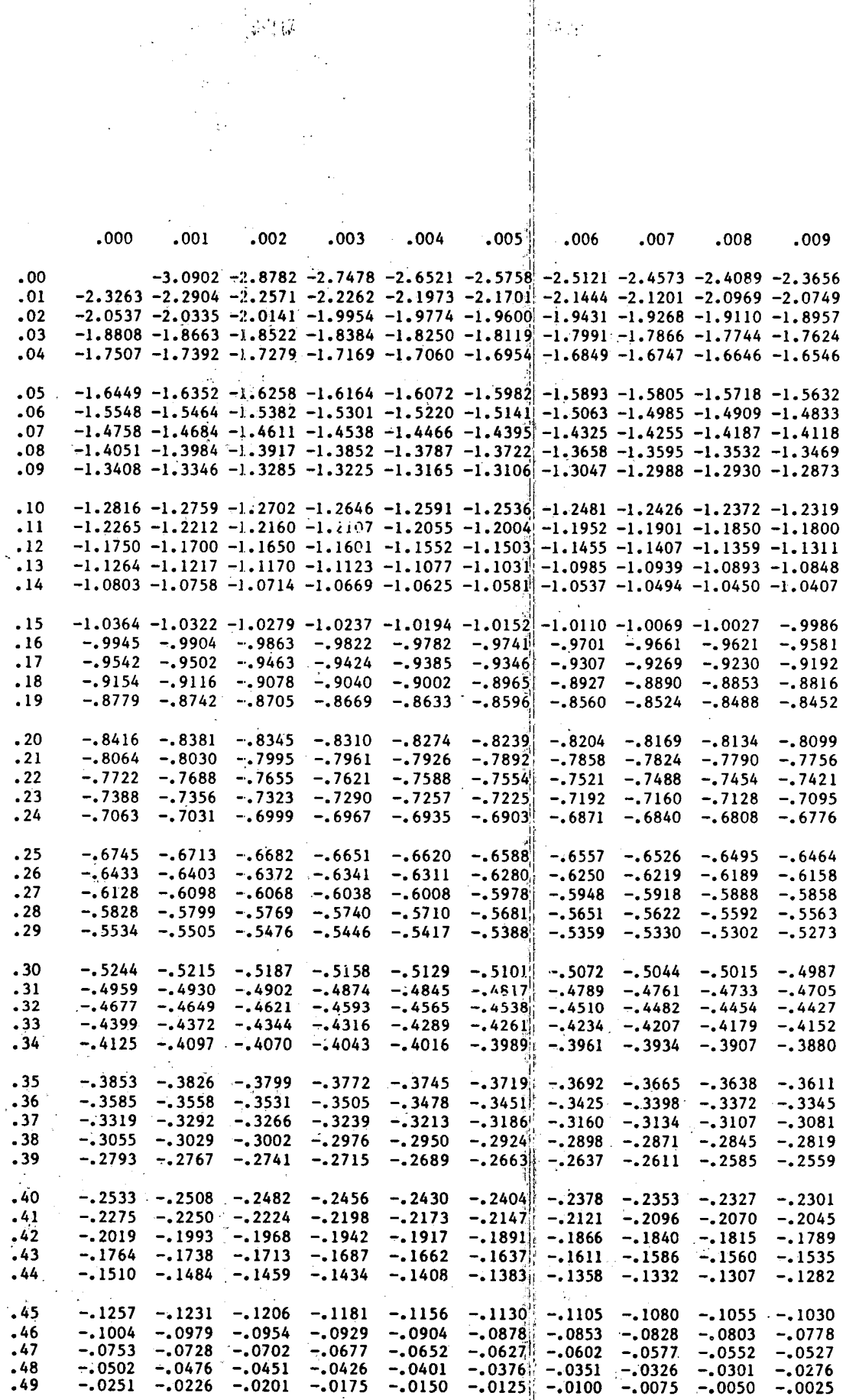

Table 2. The Cumulative Standard Noirmal Distribution 


\begin{tabular}{|c|c|c|c|c|c|c|c|c|c|c|}
\hline & .000 & .001 & .002 & .003 & .004 & .005 & .006 & .007 & .008 & .009 \\
\hline $\begin{array}{l}.50 \\
.51 \\
.52 \\
.53 \\
.54\end{array}$ & $\begin{array}{l}.0000 \\
.0251 \\
.0502 \\
.0753 \\
.1004\end{array}$ & $\begin{array}{l}.0025 \\
.0276 \\
.0527 \\
.0778 \\
.1030\end{array}$ & $\begin{array}{l}.0050 \\
.0301 \\
.0552 \\
.0803 \\
.1055\end{array}$ & $\begin{array}{l}.0075 \\
.0326 \\
.0577 \\
.0828 \\
.1080\end{array}$ & $\begin{array}{l}.0100 \\
.0351 \\
.0602 \\
.0853 \\
.1105\end{array}$ & $\begin{array}{l}.0125 \\
.0376 \\
.0627 \\
.0878 \\
.1130\end{array}$ & $\begin{array}{l}.0150 \\
.0401 \\
.0652 \\
.0904 \\
.1156\end{array}$ & $\begin{array}{l}.0175 \\
.0426 \\
.0677 \\
.0929 \\
.1181\end{array}$ & $\begin{array}{l}.0201 \\
.0451 \\
.0702 \\
.0954 \\
.1206\end{array}$ & $\begin{array}{l}.0226 \\
.0476 \\
.0728 \\
.0979 \\
.1231\end{array}$ \\
\hline $\begin{array}{r}.55 \\
.56 \\
.57 \\
.58 \\
.59\end{array}$ & $\begin{array}{r}.1257 \\
.1510 \\
.1764 \\
.2019 \\
.2275\end{array}$ & $\begin{array}{r}.1282 \\
.1535 \\
.1789 \\
.2045 \\
.2301\end{array}$ & $\begin{array}{r}.1307 \\
.1560 \\
.1815 \\
.2070 \\
.2327\end{array}$ & $\begin{array}{r}.1332 \\
.1586 \\
.1840 \\
.2096 \\
.2353\end{array}$ & $\begin{array}{r}.1358 \\
.1611 \\
.1866 \\
.2121 \\
.2378\end{array}$ & $\begin{array}{r}.1383 \\
.1637 \\
.1891 \\
.2147 \\
.2404\end{array}$ & $\begin{array}{r}.1408 \\
.1662 \\
.1917 \\
.2173 \\
.2430\end{array}$ & $\begin{array}{r}.1434 \\
.1687 \\
.1942 \\
.2198 \\
.2456\end{array}$ & $\begin{array}{r}.1459 \\
.1713 \\
.1968 \\
.2224 \\
.2482\end{array}$ & $\begin{array}{l}.1484 \\
.1738 \\
.1993 \\
.2250 \\
.2508\end{array}$ \\
\hline $\begin{array}{l}.60 \\
.61 \\
.62 \\
.63 \\
.64\end{array}$ & $\begin{array}{l}.2533 \\
.2793 \\
.3055 \\
.3319 \\
.3585\end{array}$ & $\begin{array}{r}.2559 \\
.2819 \\
.3081 \\
.3345 \\
.3611\end{array}$ & $\begin{array}{r}.2585 \\
.2845 \\
.3107 \\
.3372 \\
.3638\end{array}$ & $\begin{array}{r}.2611 \\
.2871 \\
.3134 \\
.3398 \\
.3665\end{array}$ & $\begin{array}{r}.2637 \\
.2898 \\
.3160 \\
.3425 \\
.3692\end{array}$ & $\begin{array}{r}.2663 \\
.2924 \\
.3186 \\
.3451 \\
.3719\end{array}$ & $\begin{array}{r}.2689 \\
.2950 \\
.3213 \\
.3478 \\
.3745\end{array}$ & $\begin{array}{r}.2715 \\
.2976 \\
.3239 \\
.3505 \\
.3772\end{array}$ & $\begin{array}{r}.2741 \\
.3002 \\
.3266 \\
.3531 \\
.3799\end{array}$ & $\begin{array}{r}.2767 \\
.3029 \\
.3292 \\
.3558 \\
.3826\end{array}$ \\
\hline $\begin{array}{l}.65 \\
.66 \\
.67 \\
.68 \\
.69\end{array}$ & $\begin{array}{l}.3853 \\
.4125 \\
.4399 \\
.4677 \\
.4959\end{array}$ & $\begin{array}{r}.3880 \\
.4152 \\
.4427 \\
.4705 \\
.4987\end{array}$ & $\begin{array}{r}.3907 \\
.4179 \\
.4454 \\
.4733 \\
.5015\end{array}$ & $\begin{array}{r}.3934 \\
.4207 \\
.4482 \\
.4761 \\
.5044\end{array}$ & $\begin{array}{r}.3961 \\
.4234 \\
.4510 \\
.4789 \\
.5072\end{array}$ & $\begin{array}{r}.3989 \\
.4261 \\
.4538 \\
.4817 \\
.5101\end{array}$ & $\begin{array}{r}.4016 \\
.4289 \\
.4565 \\
.4845 \\
.5129\end{array}$ & $\begin{array}{r}.4043 \\
.4316 \\
.4593 \\
.4874 \\
.5158\end{array}$ & $\begin{array}{r}.4070 \\
.4344 \\
.4621 \\
.4902 \\
.5187\end{array}$ & $\begin{array}{l}.4097 \\
.4372 \\
.4649 \\
.4930 \\
.5215\end{array}$ \\
\hline $\begin{array}{l}.70 \\
.71 \\
.72 \\
.73 \\
.74\end{array}$ & $\begin{array}{r}.5244 \\
.5534 \\
.5828 \\
.6128 \\
.6433\end{array}$ & $\begin{array}{r}.5273 \\
.5563 \\
.5858 \\
.6158 \\
.6464\end{array}$ & $\begin{array}{r}.5302 \\
.5592 \\
.5888 \\
.6189 \\
.6495\end{array}$ & $\begin{array}{r}.5330 \\
.5622 \\
.5918 \\
.6219 \\
.6526\end{array}$ & $\begin{array}{r}.5359 \\
.5651 \\
.5948 \\
.6250 \\
.6557\end{array}$ & $\begin{array}{r}.5388 \\
.5681 \\
.5978 \\
.6280 \\
.6588\end{array}$ & $\begin{array}{r}.5417 \\
.5710 \\
.6008 \\
.6311 \\
.6620\end{array}$ & $\begin{array}{r}.5446 \\
.5740 \\
.6038 \\
.6341 \\
.6651\end{array}$ & $\begin{array}{r}.5476 \\
.5769 \\
.6068 \\
.6372 \\
.6682\end{array}$ & $\begin{array}{l}.5505 \\
.5799 \\
.6098 \\
.6403 \\
.6713\end{array}$ \\
\hline $\begin{array}{l}.75 \\
.76 \\
.77 \\
.78 \\
.79\end{array}$ & $\begin{array}{l}.6745 \\
.7063 \\
.7388 \\
.7722 \\
.8064\end{array}$ & $\begin{array}{r}.6776 \\
.7095 \\
.7421 \\
.7756 \\
.8099\end{array}$ & $\begin{array}{l}.6808 \\
.7128 \\
.7454 \\
.7790 \\
.8134\end{array}$ & $\begin{array}{l}.6840 \\
.7160 \\
.7488 \\
.7824 \\
.8169\end{array}$ & $\begin{array}{l}.6871 \\
.7192 \\
.7521 \\
.7858 \\
.8204\end{array}$ & $\begin{array}{r}.6903 \\
.7225 \\
.7554 \\
.7892 \\
.8239\end{array}$ & $\begin{array}{l}.6935 \\
.7257 \\
.7588 \\
.7926 \\
.8274\end{array}$ & $\begin{array}{l}.6967 \\
.7290 \\
.7621 \\
.7961 \\
.8310\end{array}$ & $\begin{array}{r}.6999 \\
.7323 \\
.7655 \\
.7995 \\
.8345\end{array}$ & $\begin{array}{l}.7031 \\
.7356 \\
.7688 \\
.8030 \\
.8381\end{array}$ \\
\hline $\begin{array}{l}.80 \\
.81 \\
.82 \\
.83 \\
.84\end{array}$ & $\begin{array}{r}.8416 \\
.8779 \\
.9154 \\
.9542 \\
.9945\end{array}$ & $\begin{array}{r}.8452 \\
.8816 \\
.9192 \\
.9581 \\
.9986\end{array}$ & $\begin{array}{r}.8488 \\
.8853 \\
.9230 \\
.9621 \\
1.0027\end{array}$ & $\begin{array}{r}.8524 \\
.8890 \\
.9269 \\
.9661 \\
1.0069\end{array}$ & $\begin{array}{r}.8560 \\
.8927 \\
.9307 \\
.9701 \\
1.0110\end{array}$ & $\begin{array}{r}.8596 \\
.8965 \\
.9346 \\
.9741 \\
1.0152\end{array}$ & $\begin{array}{r}.8633 \\
.9002 \\
.9385 \\
.9782 \\
1.0194\end{array}$ & $\begin{array}{r}.8669 \\
.9040 \\
.9424 \\
.9822 \\
1.0237\end{array}$ & $\begin{array}{r}.8705 \\
.9078 \\
.9463 \\
.9863 \\
1.0279\end{array}$ & $\begin{array}{r}.8742 \\
.9116 \\
.9502 \\
.9904 \\
1.0322\end{array}$ \\
\hline $\begin{array}{l}.85 \\
.86 \\
.87 \\
.88 \\
.89\end{array}$ & $\begin{array}{l}1.0364 \\
1.0803 \\
1.1264 \\
1.1750 \\
1.2265\end{array}$ & $\begin{array}{l}1.0407 \\
1.0848 \\
1.1311 \\
1.1800 \\
1.2319\end{array}$ & $\begin{array}{l}1.0450 \\
1.0893 \\
1.1359 \\
1.1850 \\
1.2372\end{array}$ & $\begin{array}{l}1.0494 \\
1.0939 \\
1.1407 \\
1.1901 \\
1.2426\end{array}$ & $\begin{array}{l}1.0537 \\
1.0985 \\
1.1455 \\
1.1952 \\
1.2481\end{array}$ & $\begin{array}{l}1.0581 \\
1.1031 \\
1.1503 \\
1.2004 \\
1.2536\end{array}$ & $\begin{array}{l}1.0625 \\
1.1077 \\
1.1552 \\
1.2055 \\
1.2591\end{array}$ & $\begin{array}{l}1.0669 \\
1.1123 \\
1.1601 \\
1.2107 \\
1.2646\end{array}$ & $\begin{array}{l}1.0714 \\
1.1170 \\
1.1650 \\
1.2160 \\
1.2702\end{array}$ & $\begin{array}{l}1.0758 \\
1.1217 \\
1.1700 \\
1.2212 \\
1.2759\end{array}$ \\
\hline $\begin{array}{l}.90 \\
.91 \\
.92 \\
.93 \\
.94\end{array}$ & $\begin{array}{l}1.2816 \\
1.3408 \\
1.4051 \\
1.4758 \\
1.5548\end{array}$ & $\begin{array}{l}1.2873 \\
1.3469 \\
1.4118 \\
1.4833 \\
1.5632\end{array}$ & $\begin{array}{l}1.2930 \\
1.3532 \\
1.4187 \\
1.4909 \\
1.5718\end{array}$ & $\begin{array}{l}1.2988 \\
1.3595 \\
1.4255 \\
1.4985 \\
1.5805\end{array}$ & $\begin{array}{l}1.3047 \\
1.3658 \\
1.4325 \\
1.5063 \\
1.5893\end{array}$ & $\begin{array}{l}1.3106 \\
1.3722 \\
1.4395 \\
1.5141 \\
1.5982\end{array}$ & $\begin{array}{l}1.3165 \\
1.3787 \\
1.4466 \\
1.5220 \\
1.6072\end{array}$ & $\begin{array}{l}1.3225 \\
1.3852 \\
1.4538 \\
1.5301 \\
1.6164\end{array}$ & $\begin{array}{l}1.3285 \\
1.3917 \\
1.4611 \\
1.5382 \\
1.6258\end{array}$ & $\begin{array}{l}1.3346 \\
1.3984 \\
1.4684 \\
1.5464 \\
1.6352\end{array}$ \\
\hline $\begin{array}{r}.95 \\
.96 \\
.97 \\
.98 \\
.99\end{array}$ & $\begin{array}{l}1.6449 \\
1.7507 \\
1.8808 \\
2.0537 \\
2.3263\end{array}$ & $\begin{array}{l}1.6546 \\
1.7624 \\
1.8957 \\
2.0749 \\
2.3656\end{array}$ & $\begin{array}{l}1.6646 \\
1.7744 \\
1.9110 \\
2.0969 \\
2.4089\end{array}$ & $\begin{array}{l}1.6747 \\
1.7866 \\
1.9268 \\
2.1201 \\
2.4573\end{array}$ & $\begin{array}{l}1.6849 \\
1.7991 \\
1.9431 \\
2.1444 \\
2.5121\end{array}$ & $\begin{array}{l}1.6954 \\
1.8119 \\
1.9600 \\
2.1701 \\
2.5758\end{array}$ & $\begin{array}{l}1.7060 \\
1.8250 \\
1.9774 \\
2.1973 \\
2.6521\end{array}$ & $\begin{array}{l}1.7169 \\
1.8384 \\
1.9954 \\
2.2262 \\
2.7478\end{array}$ & $\begin{array}{l}1.7279 \\
1.8522 \\
2.0141 \\
2.2571 \\
2.8782\end{array}$ & $\begin{array}{l}1.7392 \\
1.8663 \\
2.0335 \\
2.2904 \\
3.0902\end{array}$ \\
\hline
\end{tabular}


The Purpose of the Course

This is a tutorial on regression methods. It introduces and discusses the topics of regression on one variable, rank regression on one variable, and then proceeds to the case of regression on several variables, using raw data or ranks. Stepwise regression is discussed as a means of selecting important variables. Other regression procedures known as forward regression and backward regression are also mentioned. At the conclusion of this tutorial the reader should be able to understand better the regression program described in "Stepwise Regression with PRESS and Rank Regression (Program Users Guide)" by Iman, Davenport, Frost and Shortencarier (1980).

The Need for Regression Methods

Regression methods are useful for identifying and/or defining the relationship between a variable of interest $Y$ and one or more observable variables, called independent variables and denoted by $x_{1}, x_{2}$, etc. Although regression methods are used in a variety of ways, their primary importance on the study of geologic models is for identifying the input variables $x_{1}$, $X_{2}, \ldots$... Which are the most influential on the output variable $Y$. For this specific goal of identification of important variables, some regression methods are particularly useful. These include rank regression and stepwise regression. To lead into these topics, simple regression is introduced first.

\section{Simple Linear Regression}

Simple regression refers to the case where only one independent variable is considered. Observations $\left(y_{1}, x_{1}\right),\left(y_{2}, x_{2}\right), \ldots$, on the bivariate random variable $(Y, X)$ are analyzed to see what type of relationship may exist between $Y$ and $X$. In all but artificial situations, the exact relationship between $Y$ and $X$ cannot be expressed mathematically, so regression methods are directed toward approximating the exact relationship between $Y$ and $X$ with mathematical equations. The simpliest mathematical equation is a straight line, so a straight $l$ ine is the most popular equation to fit to a set of points such as the observations $\left(y_{1}, x_{1}\right),\left(y_{2}, x_{2}\right), \ldots,\left(y_{n}, x_{n}\right)$. The equation of a straight line is

$$
y=a+b x
$$

The analysis using linear regression begins with finding estimates $\hat{a}$ and $\vec{b}$ so that the straight line will agree well with the data. The most popuTar method of finding $\underline{\underline{a}}$ and $\underline{\hat{b}}$ is called the method of least squares.

The Method of Least Squares

When an estimated regression equation is obtained, the observed values of $X$ may be substituted into the regression equation to get values of $Y$ which are called predicted values of $Y$ and are denoted by $Y$. In the case 
of simple linear regression the predicted vallues are given by

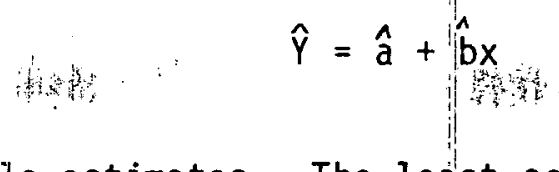

where $\hat{a}$ and $\hat{b}$ are sample estimates. The leàst squares method for finding $\underline{a}$ and $\underline{b}$ chooses the numbers that minimize the sum of squares

$$
S S=\Sigma(Y-\hat{Y})^{2}
$$

This method assures that the predicted values $\hat{Y}$ will be as close as possible (in the least squares sense) to the observed values $Y$.

\section{The Least Squares Equations:}

In this case of one independent variable the least squares solutions for $\underline{\underline{a}}$ and $\underline{b}$ are simple to express

$$
\begin{aligned}
& \hat{b}=\frac{\sum_{i=1}^{n} x_{i} y_{i}-\left(\Sigma x_{i}\right)\left(\Sigma y_{i}\right) / n}{\sum_{i=1}^{n} x_{i}^{2}-\left(\Sigma x_{i}\right)^{2} / n} \\
& \hat{a}=\frac{1}{n}\left(\Sigma y_{i}-b \Sigma x_{j}\right)
\end{aligned}
$$

and may be computed on a hand calculator. When there are two or more independent variables the calculations become much more difficult and are usually performed on a computer.

\section{Example}

A simple example is used to illustrate the computations involved in the least squares solution to simple linearilregression. The same example will then be used to introduce rank regression. Suppose five observations on $(Y, X)$ are obtained as given in Figure 1.4 The least squares coefficients are found using Equations (1) and (2).

$$
\begin{aligned}
& \hat{b}=\frac{199.83-(29.0)(28.4) / 5}{189.94-(28.4)^{2} / 5}=\frac{35.110}{28.628}=1.226 \\
& \hat{a}=\frac{1}{5}(29.0-(1.226)(28.4))=-1.164
\end{aligned}
$$


A graph of the five observations and the least squares regression line

$$
\hat{Y}=-1.164+1.226 X
$$

are given in Figure 2 .

\begin{tabular}{crrrr}
$\begin{array}{crrr}\text { Obs. } \\
\text { Pair }\end{array}$ & $Y$ & $X$ & \multicolumn{1}{c}{$X^{2}$} & \multicolumn{1}{c}{$X Y$} \\
\cline { 1 - 1 } 1 & 1.4 & 2.3 & 5.29 & 3.22 \\
2 & 5.3 & 4.1 & 16.81 & 21.73 \\
3 & 4.8 & 5.6 & 31.36 & 26.88 \\
4 & 6.5 & 7.2 & 51.84 & 46.80 \\
5 & $\frac{11.0}{29.0}$ & $\frac{9.2}{28.4}$ & $\frac{84.64}{189.94}$ & $\frac{101.20}{199.83}$
\end{tabular}

Figure 1. Worksheet for Finding $\underline{\hat{a}}$ and $\underline{\hat{b}}$ Using Least Squares.

The linear regression model appears to fit the points in Figure 2 fairly well. The residuals $(Y-Y)$ are a measure of how well the linear regression model agrees with the data points

$$
\text { residual }=Y-\hat{Y}=Y-(-1.164+1.226 X)
$$

This choice of coefficients, $\hat{a}=-1.164$ and $\hat{b}=1.226$, results in the smallest possible sum of squares achievable using a straight line to fit the

\begin{tabular}{|c|c|c|c|c|c|}
\hline $\begin{array}{l}\text { Obs. } \\
\text { Pair }\end{array}$ & $Y$ & $x$ & $\hat{Y}$ & Residual & $(Y-\hat{Y})^{2}$ \\
\hline 1 & 1.4 & 2.3 & 1.6558 & -0.2558 & .0654 \\
\hline 2 & 5.3 & 4.1 & 3.8626 & 1.4374 & 2.0661 \\
\hline 3 & 4.8 & 5.6 & 5.7016 & -0.9016 & .8129 \\
\hline 4 & 6.5 & 7.2 & 7.6632 & -1.1632 & 1.3530 \\
\hline 5 & 11.0 & 9.2 & 10.1152 & 0.8848 & $\frac{.7829}{5,0803}$ \\
\hline
\end{tabular}
data. In this case the minimum value is $S S=5.0803$, as given in Figure 3 .

Figure 3. The Residuals and Sum of Squares from Figure 2.

Rank Regression

For the set of data given in Figure 1 the 1 inear regression model appears to be satisfactory, so no further analysis would usually be required. However, merely for the sake of illustration, rank regression 


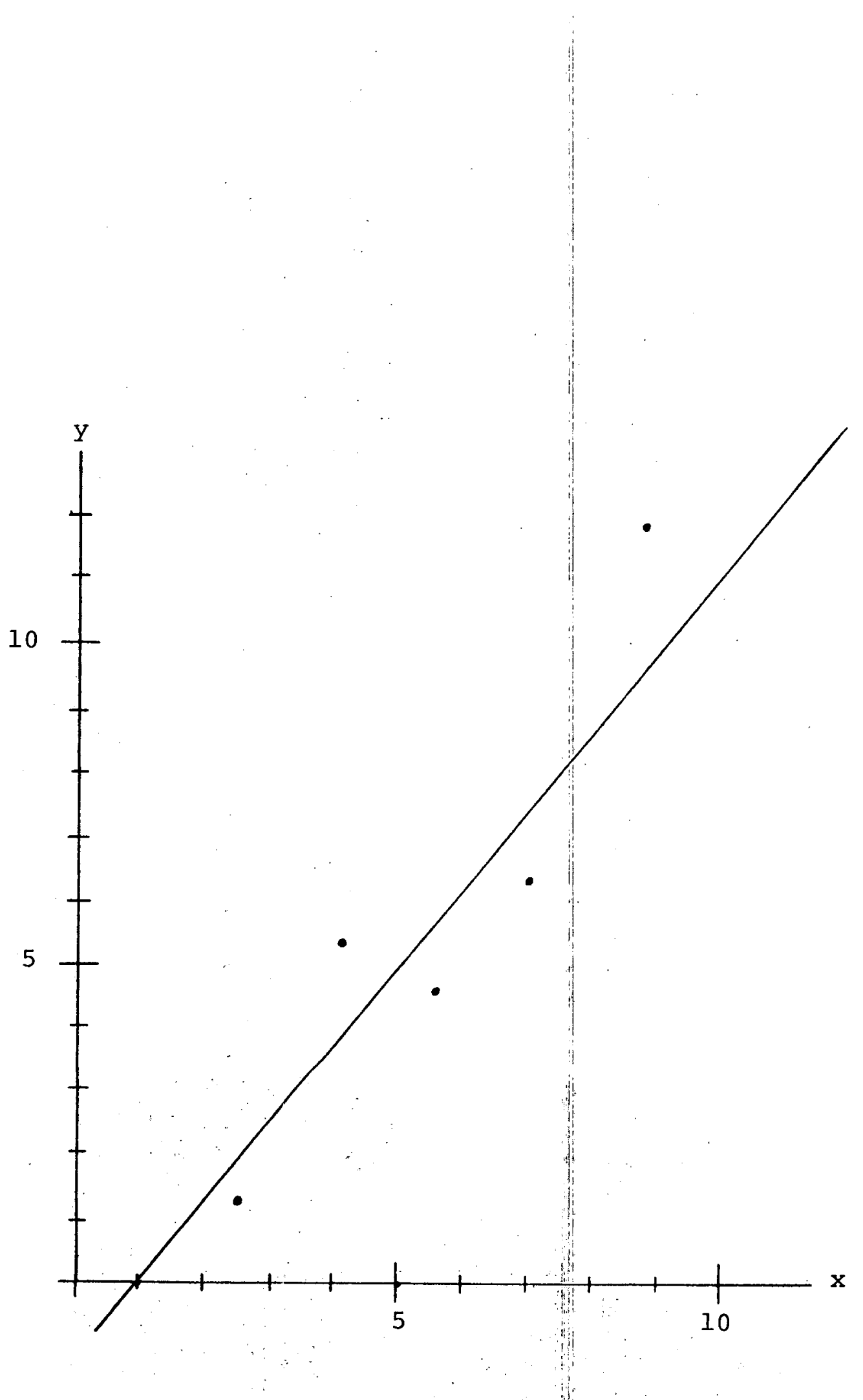

Figure 2. A Graph of the Data in Figure 1, and the Least Squares Regression Line. 
is used on the same set of data and comparisons are made with regression on the raw data.

Rank regression involves simply the usual regression methods appl ied to the ranks of the data rather than to the data themselves. The smallest observation has rank 1, the second smallest has rank 2, and so on to the largest of $n$ observations which has rank $n$. In case several observations in a group are all exactly equal to each other (tied), the rank assigned to each is the average of the ranks that would have been assigned to them had they not been tied. This is called the average ranks method of handling ties. In rank regression each variable is ranked by itself; that is, the observations on $Y$ are ranked separately from the observations on $X_{1}$, which are in turn ranked separately from the observations on $x_{2}$, and so on. The ranks of the data in Figure 1 are given in Figure 4 . Note that the ranks $r_{y}$ of the $Y$ 's are obtained independently of the ranks $r_{X}$ of the $X^{\prime} s$.

The least squares equation on the ranks is obtained using Equations (1) and (2) just as on the original data.

$$
\begin{aligned}
\hat{b}_{r}= & \Sigma r_{x} r_{y}-\left(\Sigma r_{x}\right)\left(\Sigma r_{y}\right) / n \\
& \Sigma r_{x}^{2}-\left(\Sigma r_{x}\right)^{2} / n \\
= & \frac{54-(15)(15) / 5}{55-(15)^{2} / 5}=\frac{9}{10}=.9 \\
\hat{a}_{r}= & \frac{1}{n}\left(\Sigma r_{y}-b \Sigma r_{x}\right) \\
= & \frac{1}{5}(15-(.9)(15))=.3
\end{aligned}
$$

Therefore the least squares equation on the ranks is given by

$$
\begin{aligned}
\hat{r}_{y} & =\hat{a}_{r}+\hat{b}_{r} r_{x} \\
& =.3+.9 r_{x}
\end{aligned}
$$

$\begin{array}{rrrrrrr}\text { Obs. } & & & & & \\ \text { Pair } & Y & X & r_{y} & r_{x} & r_{x}{ }^{2} & r_{x} r_{y} \\ 1 & 1.4 & 2.3 & 1 & 1 & 1 & 1 \\ 2 & 5.3 & 4.1 & 3 & 2 & 4 & 6 \\ 3 & 4.8 & 5.6 & 2 & 3 & 9 & 6 \\ 4 & 6.5 & 7.2 & 4 & 4 & 16 & 16 \\ 5 & 11.0 & 9.2 & \underline{5} & \underline{5} & \underline{25} & \underline{25} \\ \text { Total } & & & 15 & 15 & 55 & 54\end{array}$

Figure 4. Worksheet for Least Squares on the Ranks 


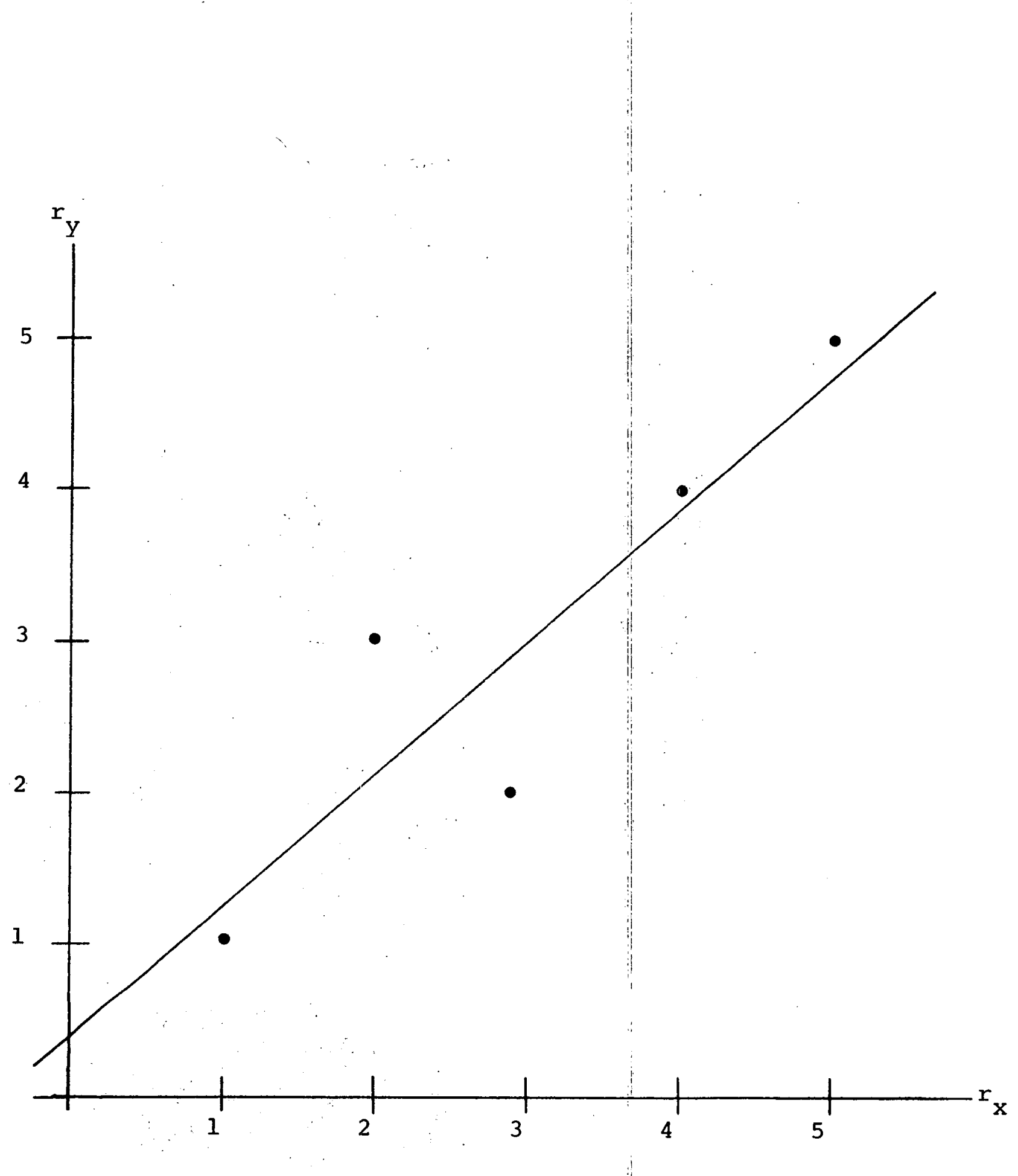

Figure 5. A Graph of the Ranks from Figure 4 and the Least Squares Line on the Ranks. 
A graph of the ranks of the data, and the least squares straight 1 ine computed for the ranks is given in Figure 5. Again note that the 1 inear model fits the ranks fairly well, just as the linear model fit the data fairly well in Figure 2. This is a typical relationship between rank regression and ordinary regression. If the simple linear regression model fits the data well, it usually works well on the ranks al so. However, rank regression is useful in situations where simple linear regression does not work satisfactorily with the data. This point will be illustrated in a later example, but first the residuals from the rank regression procedure will be computed.

\section{Converting Predicted Ranks to Predicted Values}

There are two types of residuals from rank regression. One type of residual is the difference between the predicted ranks of $Y$ and the actual rank of $Y$, which is obtained in the same way $P$ was obtained in ordinary regression, as in Figure 2, but using ranks $r_{y}$ and $r_{X}$ instead of $Y$ and $X$, and using the equation for ranks Equation (5) instead of the least squares line for the data. These are called rank residuals and are not useful because they convey no information on how welt the data are being fitted, only information on how well the ranks are being fitted.

To see how well the data are being fitted, the predicted rank $\hat{r}_{y}$ of each observation $Y$ is obtained from Equation, (5). These predicted ranks are converted to predicted values $\hat{Y}$ of $Y$ by comparing the predicted ranks with the actual ranks of the five observations on $Y$, and obtaining predicted values of $Y$ on the basis of this comparison, using interpolation if necessary.

$\begin{array}{cccccccc}\begin{array}{c}\text { Obs. } \\ \text { Pair }\end{array} & r_{y} & r_{X} & \hat{r}_{y} & \hat{Y} & Y & \text { Residual } & (Y-\hat{Y})^{2} \\ 1 & 1 & 1 & 1.2 & 2.08 & 1.4 & .68 & .4624 \\ 2 & 3 & 2 & 2.1 & 4.85 & 5.3 & -.45 & .2025 \\ 3 & 2 & 3 & 3.0 & 5.30 & 4.8 & .50 & .2500 \\ 4 & 4 & 4 & 3.9 & 6.38 & 6.5 & -.12 & .0144 \\ 5 & 5 & 5 & 4.8 & 10.10 & 11.0 & -.90 & .8100 \\ \text { Total } & & & & & & & \text { SS }=1.7393\end{array}$

Figure 6. Worksheet for Finding Residuals from Rank Regression 


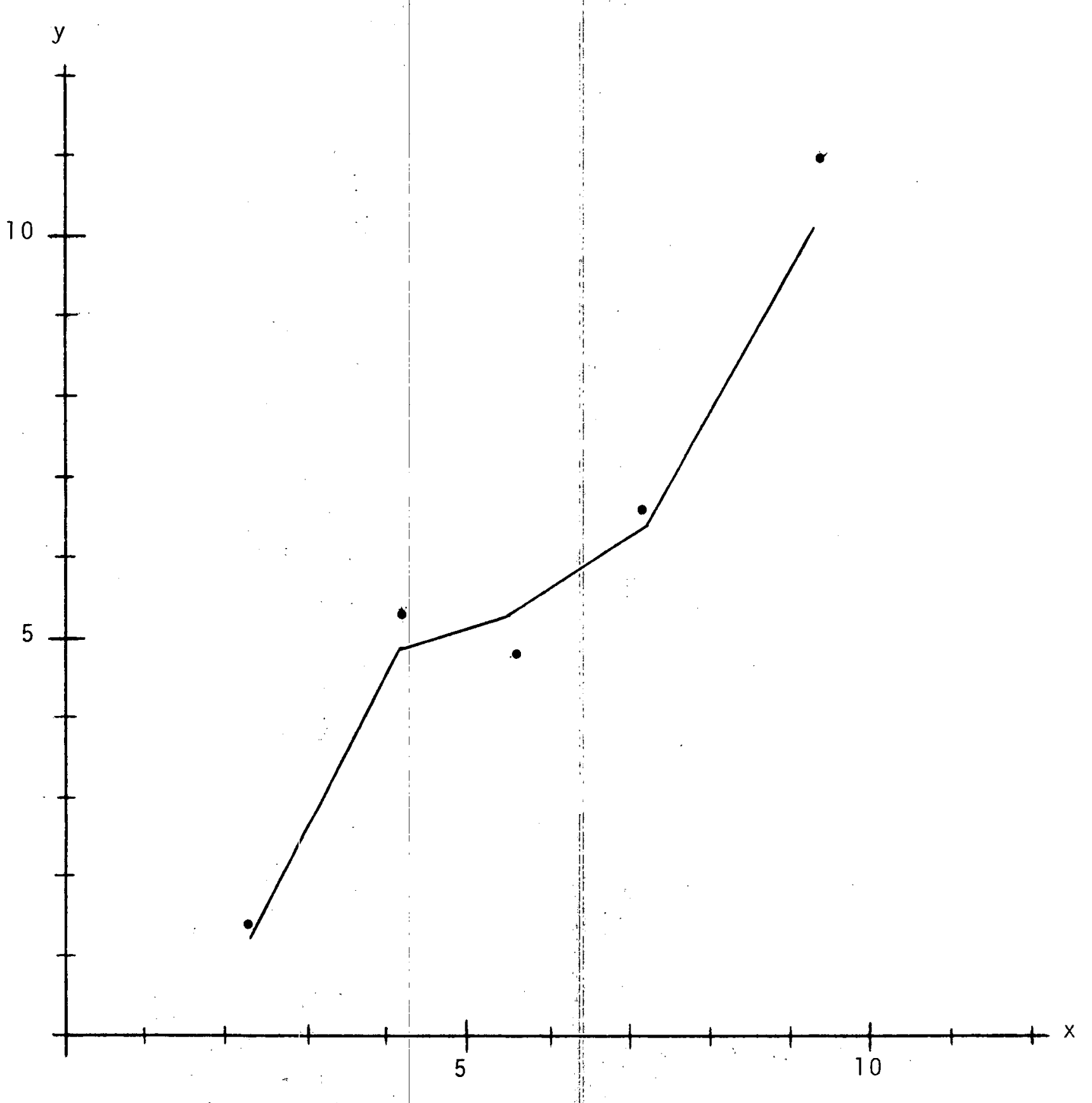

Figure 7: A graph of the Datal in Figure 1, and the Rank Regression Curve 
For example, to find the $\hat{Y}$ corresponding to the first pair of observations $\left(y_{1}, x_{1}\right)=(1.4,2.3)$, enter the rank of $x_{1}, r_{x}=1$, into Equation (5) to get the predicted rank of $y_{1}$;

$$
\begin{aligned}
\hat{r}_{y} & =.3+.9 r_{x} \\
& =.3+(.9)(1) \\
& =1.2
\end{aligned}
$$

The predicted $Y$ corresponding to a predicted rank 1.2 is found by interpolating between the actual observed $Y$ with rank $1, Y=1.4$, and the actual observed $Y$ with rank $2, Y=4.8$;

$$
\hat{Y}=1.4+\frac{1.2-1.0}{2.0-1.0}(4.8-1.4)=2.08
$$

This predicted value $\hat{Y}=2.08$ is compared with the observed value in the first pair $Y=1.4$ to get the residual 0.68 . A summary of the calculations, including the residuals and the sum of squares is given in Figure 6 .

\section{The Residuals Sum of Squares (SS)}

Note that the residuals in Figure 6 tend to be smaller than the residuals found from the least squares fit to the original data, given in Figure 3. The sum of squares from Figure 6

$$
\text { SS }=1.7393 \quad \text { (Rank Regression) }
$$

is much smaller than the sum of squares from Figure 3.

$$
S S=5.0803 \quad \text { (Regression on Data) }
$$

The residuals sum of squares from Figure 3 is the smallest that can be obtained from a straight line fit to the data. But rank regression does not give a straight 1 ine fit to the data. To show this, a graph of all possible rank regression predictions is given in Figure 7 . Note that the rank regression equation adapts itself to the points observed, but is steadily increasing as $x$ increases. Rank regression equations are monotonically increasing or decreasing, and therefore work very well with data that tend to show a monotonic relationship, even though the relationship may be nonlinear. 
Exercise 1

Use the following steps to find the predicted value $\hat{y}=4.85$, for the second pair of observations $\left(y_{2}, x_{2}\right)=(5.3,4.1)$, as given in Figure 6 from rank regression. See Figure 4 for the original data.

1. Find the rank of $x_{2}=4.1$.

2. Substitute the rank of $x_{2}$ into Equation (5) to get a predicted rank for $y_{2}$. Compare with the value from Figure 6 .

3. Find the two observed values of $Y$ whose ranks straddle (just above and just below) the predicted rank for $y_{2}$.

4. Interpolate between the two observed values for $Y$ from step 3 , to get a predicted value for $y_{2}$. that correspond's to the predicted rank for $y_{2}$ from step 2. This predicted value should match the value $\hat{\gamma}=4.85$ from $F$ igure 6 .

The Flexibility of Rank Regression for Fitting Monotonic Data

The ability of the rank regression curve to adapt to a set of points which exhibit a nonl inear, but monotonic relationship. is shown more dramatically in Figure 9. In Figure 8 nine points are obtained from the equation $y=e^{x}$, with no error of measurement added. The basic premise here is that a good regression technique should work well if the conditions are ideal. Here the conditions for rank regression are ideal, since the relationship between $X$ and $Y$ is monotonic. Because of this monotonic relationship between $X$ and $Y$, the ranks of $X$ show an exactly linear relationship with the ranks of $Y$. That is, the smallest $X$ is paired with the smallest $Y$, so rank 1 for $X$ is paired with rank 1 for $Y$. The second smallest $X$ is paired with the second smallest $Y$ so rank 2 for $X$ is plotted against rank 2 for $Y$, and so on for all of the ranks. The ranks for the data in Figure 8 are graphed in Figure 10. This results in a rank regression equation in Figure 9 which consists of a series of line segments connecting the observed points. The fit is excellent. The least squares straight line is shown also, to dramatize the 1 imitations of that method.

An Example with Real Data

The first two examples both involve artificial data. The first example serves merely to introduce the methodology of simple linear regression and rank regression. The second example illustrates a monotonic relationship between $X$ and $Y$, and shows the ability of rank regression to adapt to data of this type. A third example will now be presented. It involves real data, where the independent variable $X$ represents chemical measurements obtained using a relatively inexpensive titration method, and the dependent variable $Y$. represents corresponding measurements obtained by a more expensive extraction and weighing technique. Twenty samples were obtained, and each sample was thoroughly mixed just before being split and analyzed by both methods.

This set of data is presented and thoroughly lanalyzed by Daniel and Wood (1971). Other authors have used these data in their papers on new regression methods, so this set of data is now a classical standard on which 


$\begin{array}{rrrrrrrrrr}\mathrm{X}: & -2 & -1.5 & -1 & -0.5 & 0 & 0.5 & 1 & 1.5 & 2 \\ \mathrm{Y}:=\mathrm{e}^{\mathrm{X}}: & .14 & .22 & .37 & .61 & 1.00 & 1.65 & 2.72 & 4.48 & 7.39\end{array}$

Figure 8. Nine Values from the Function $Y=e^{x}$.

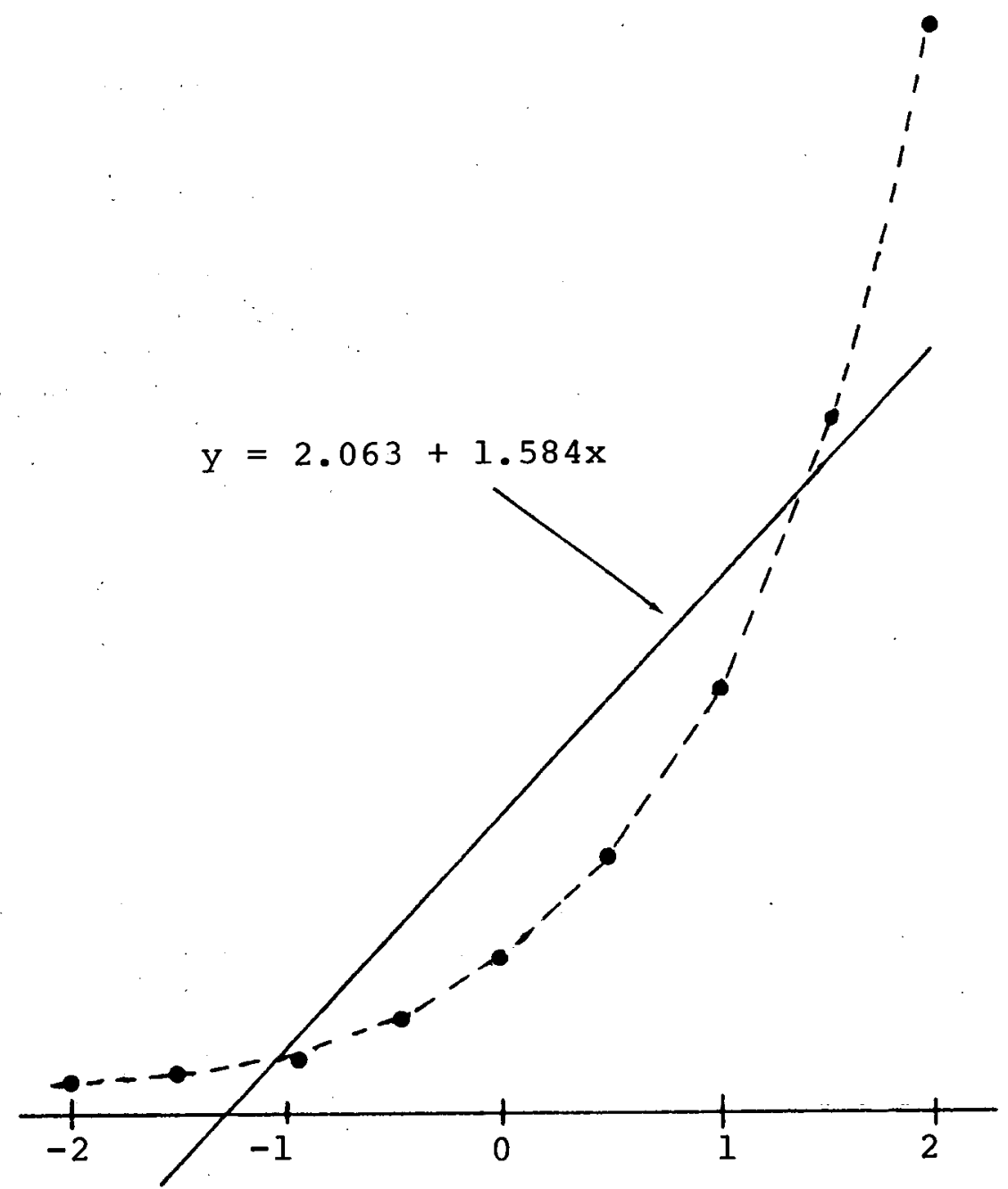

Figure 9. Rank Regression Curve and the Least Square Line for 9 Points from the Relationship $Y=e^{X}$. 


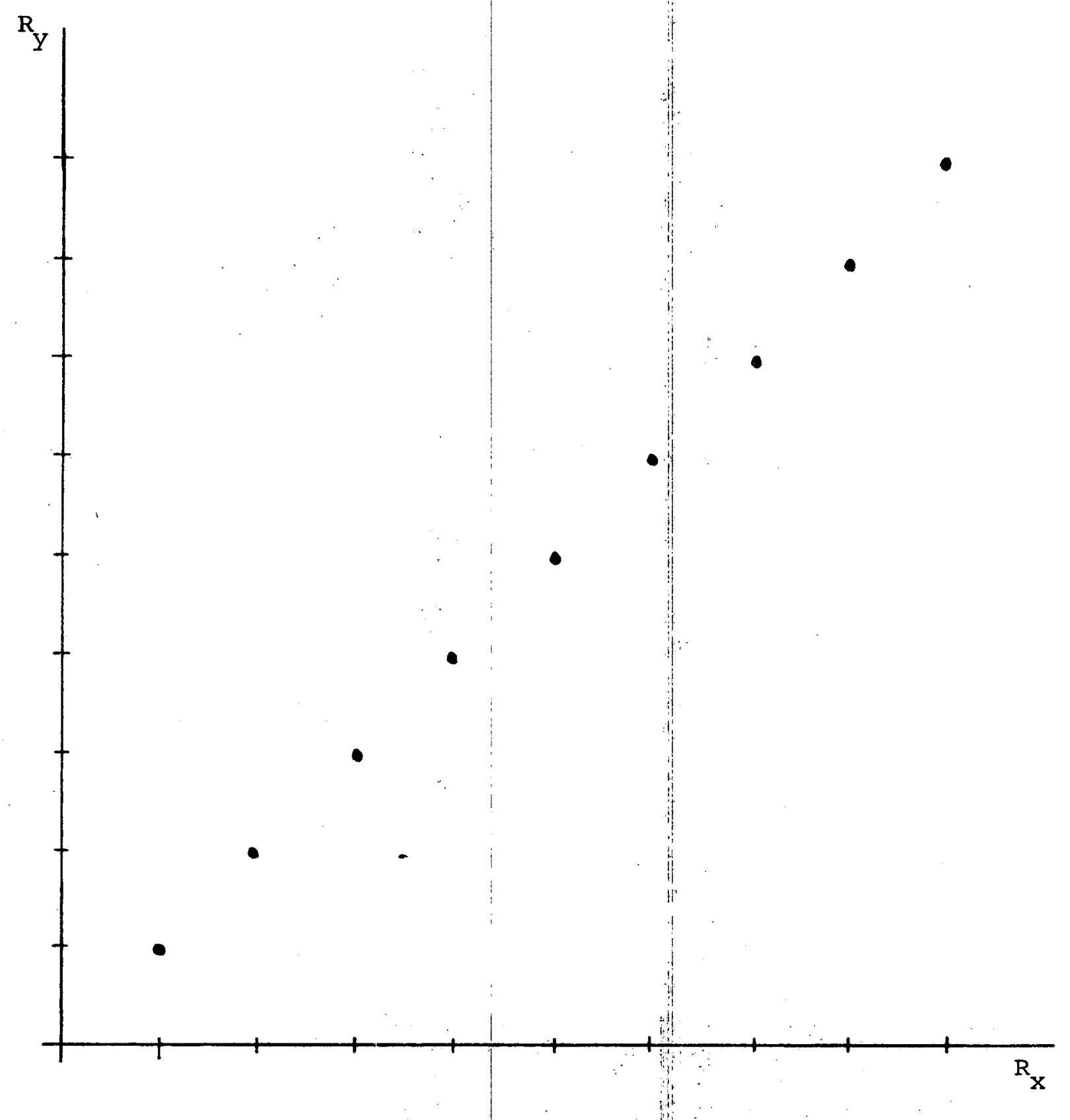

Figure 10. Graph of the Ranks of $X$ and $Y$ as Given in Figure 9 . 
new regression methods are tested. The data are somewhat unrealistic in that they follow a linear relationship very closely, with a correlation coefficient of .997. Most bivarjate data sets encountered in appl ied work have a smaller correlation, are somewhat nonl inear in appearance, and contain occasional outliers which cannot be discarded because they represent legitimate measurements. Rank regression is more suited to the messy types of data, with nonlinearities and outliers, than to this type of data which is adequately explained by ordinary linear regression. But merely for the sake of illustration, rank regression is applied to this set of data and the results are compared with the fit from ordinary regression. The data and the residuals are given in Figure 11 .

Obs.

No.

$$
\begin{aligned}
& 1 \\
& 2 \\
& 3 \\
& 4
\end{aligned}
$$$$
5
$$

$7 \quad 50$

$8 \cdot 66$

9

10

11

12

13

14

15

16

17

18

19

20

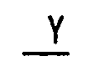

\section{6}

70

55

71

55

48

50

66

\section{1}

43

82

68

88

58

64

88

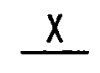

123

109

62

104

57

37

44

100

16

28

138

105

159

75

88

164

89

88

169

167

84

88

149

167

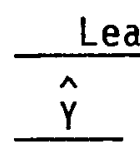

75.02

70.51

55.40

68.91

53.79

47.36

49.61

67.62

40.60

44.46

79.84

69.23

86.59

59.58

63.76

88.20

89.81

89.17

83.38

89.17

\begin{tabular}{|c|c|}
\hline$\hat{y}$ & Residual \\
\hline $\begin{array}{l}75.85 \\
70.98\end{array}$ & $\begin{array}{r}.15 \\
-.98\end{array}$ \\
\hline $\begin{array}{l}56.11 \\
68.01\end{array}$ & $\begin{array}{r}-1.11 \\
2.99\end{array}$ \\
\hline
\end{tabular}

Rank Regression

55.00

48.18

50.26

66.04

41.23

43.51

81.75

69.99

85.49

58.25

64.06

88.00

88.95

88.00

83.89

88.00

$-1.17$

$S S=27.23$

$S S=21.94$

Figure 11. A Comparison of Least Squares Linear Regression on the Data with Regression on the Ranks, Using Data from Daniel and Wood (1971). 


\section{Comparing Rank Regression With Ordinary Regression}

A comparison of the residuals in Figure 11 shows. that the residuals from the rank regression are smaller than the residuals from ordinary linear regression for 15 out of the 20 points. The sum of squares of the residuals is only 21.94 for rank regression, compared with 27.23 for ordinary 1 inear regression. The point to be made with this examplle is tht rank regression works well even with data that follow a close linear pattern.

The analysis of the data in Figure 11 was performed using the regression program described in Iman et al (1980). The slight difference in these results and the results reported in Iman and Conover (1979) is due to a difference in the method of handling ties.

\section{Multiple Regression}

The regression examples with one independent variable are useful for illustrating the principles behind ordinary regression and rank regression. When the number of independent variables is two or more these same principles apply but they become very difficult to illustrate. With two independent variables the least squares method on the data is used to fit a plane to data in the three dimensional space spanned by $Y, X_{1}$ and $X_{2}$. The rank regression method uses the least squares method to fit a plane to the ranks of the data, in the three dimensional space spanned by the ranks of $Y$, the ranks of $X_{1}$, and the ranks of $x_{2}$. When this plane is translated back to the three dimensional space spanned by $Y, X_{1}$ and $X_{2}$, the result is a series of connected mini planes that adapt to the data, in a monotonic manner, just as the series of line segments adapted to the data in the case of one independent variable. The extension to include more than two independent variables is simple in concept, but impossible to visualize because the discussion involves hyperplanes in many dimensional space.

\section{An Example of Multiple Regression}

An example is now presented which illustrates the results of ordinary multiple regression and multiple regression on the ranks. The data given in Figure 12 are from Brownlee (1965), and have become somewhat of a standard set of data for use in comparing new regression methods with old methods. They follow a linear regression pattern closely, with $\mathrm{R}^{2}=.914$. The measure of fit for a regression model is usually $R^{2}$, which states the proportion of variability of $Y$ that is explained by the regression model. An $R^{2}$ of .914 means that $91.4 \%$ of the variation in $Y$ is explained by regression on the variables $x_{1}, x_{2}$ and $x_{3}$. This figure is much closer to $100 \%$ than the $R^{2}$ values normally encountered in appl ied work.

The data in Figure 12 represent 21 successive days of operation of a plant oxidizing ammonia to nitric acid. The variables in Figure 12 are as follows:

$Y=10$ times the percentage of the ingoing ammonia that is lost as unabsorbed nitric oxides; it is an indirect measure of the yield of nitric acid.

$x_{1}=$ the flow of air to the plant 


\begin{tabular}{|c|c|c|c|}
\hline $\begin{array}{l}\text { Obs. } \\
\text { No. }\end{array}$ & $Y$ & $x_{1}$ & $x_{2}$ \\
\hline 1 & 42 & 80 & 27 \\
\hline 2 & 37 & 80 & 27 \\
\hline 3 & 37 & 75 & 25 \\
\hline 4 & 28 & 62 & 24 \\
\hline 5 & 18 & 62 & 22 \\
\hline 6 & 18 & 62 & 23 \\
\hline 7 & 19 & 62 & 24 \\
\hline 8 & 20 & 62 & 24 \\
\hline 9 & 15 & 58 & 23 \\
\hline 10 & 14 & 58 & 18 \\
\hline 11. & 14 & 58 & 18 \\
\hline 12 & 13 & 58 & 17 \\
\hline 13 & 11 & 58 & 18 \\
\hline 14 & 12 & 58 & 19 \\
\hline 15 & 8 & 50 & 18 \\
\hline 16 & 7 & 50 & 18 \\
\hline 17 & 8 & 50 & 19 \\
\hline 18 & 8 & 50 & 19 \\
\hline 19 & 9 & 50 & 20 \\
\hline 20 & 15 & 56 & 20 \\
\hline 21 & 15 & 70 & 20 \\
\hline
\end{tabular}

Figure 12. Multivariate Data From Brownlee (1965). 
Obs.

No.

1

2

3

4

5

6

7

8

9

10

11

12

13

14

15

16

17

18

19

20

21

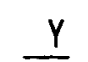

42

37

37

28

18

18

19

20

15

14

14

13

11

12

8

7.

8

8

9

15

15

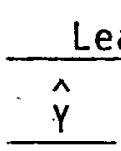

38.77

38.92

32.44

22.30

19.71

21.01

21.39

21.39

18.14

12.73

11.36

10.22

12.43

12.05

5.64

6.09

9.52

8.46

9.60

13.59

22.24 $\underline{\text { Residual }}$

3.23

$-1.92$

4.56

5.70

$-1.71$

$-3.01$

$-2.39$

$-1.39$

$-3.14$

1.27

2.64

2.78

$-1.43$

$-0.05$

2.36

.91

$-1.52$

$-0.46$

$-0.60$

1.41

$-7.24$

$S S=178.83$

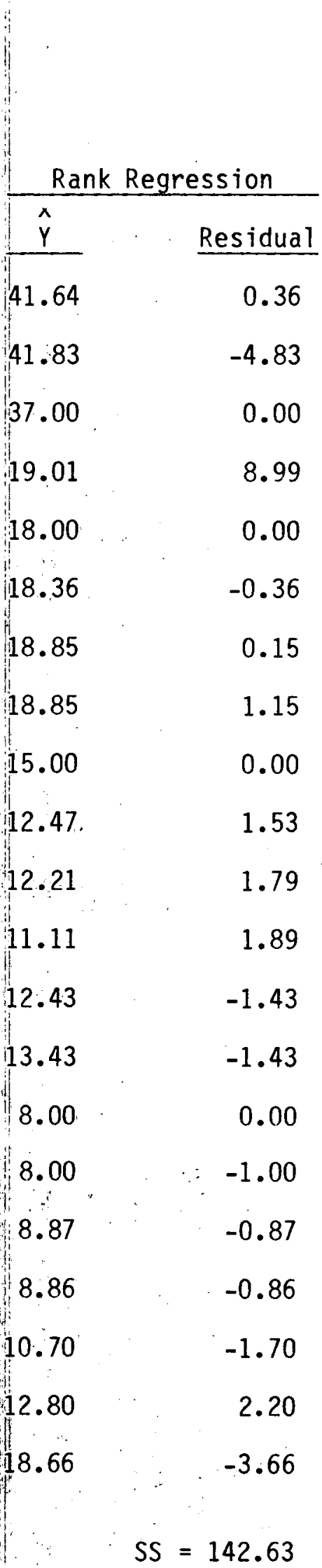

$S S=142.63$

Figure 13. Predicted Values and Residuals Using Least. Squares Regression and Rank Regression, on the Data from Figure 12, Using the Variables $x_{1}, x_{2}$ and $x_{3}$. 
$x_{2}=$ The temperature of the cooling water entering the countercurrent nitric oxide absorption tower

$x_{3}=$ the concentration of nitric acid in the absorbing liquid

Ordinary Multiple Regression Illustrated

The model used to fit the data is

$$
y=\beta_{0}+\beta_{1} x_{1}+\beta_{2} x_{2}+\beta_{3} x_{3}
$$

The method of least squares is used to find the values of $\beta_{0}, \beta_{1}, \beta_{2}$ and $\beta_{3}$ that minimize the residual sum of squares. The equations for finding the least squares coefficients are very complex and are not presented. The actual values for the coefficients appear as part of the computer printout, and are given in Equation (7).

$$
y=-39.92+.7156 x_{1}+1.2953 x_{2}-.1521 \times 3
$$

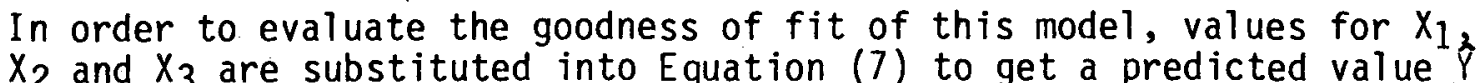
for $Y$. For example, in observation number 1 in Figure $12, X_{1}=80, X_{2}=$ 27 and $x_{3}=89$. These are substituted into Equation $(7)$ as follows,

$$
\hat{Y}=-39.92+(.7156)(80)+(1.2953)(27)-(.1521)(89)=38.76
$$

to get 38.76 as the predicted value for $Y$. This is compared with the observed value for $Y, 42$, given in Figure 12 . The residual $(42-38.76)=$ 3.24 agrees with the value given in Figure 13, except for differences caused by rounding off the coefficients in Equation (7). The sum of squares of residuals is a measure of the goodness of fit of the model. In this case it is given by

$$
\begin{aligned}
S S & =\Sigma(Y-\hat{Y})^{2} \\
& =178.83
\end{aligned}
$$

as shown in Figure 13. This is the smallest value of SS possible using the model given by Equation (6).

\section{Exercise 2}

Substitute the values for $X_{1}, X_{2}$ and $X_{3}$, given in Figure 12 under observation number 2, into Equation (7) to verify that the predicted value in Figure 13 is indeed correct. 


\section{Rank Multiple Regression Illustrated}

If the model in Equation (6) is used on the Ranks instead of the data it becomes

$$
r_{y}=\beta_{0}+\beta_{1} r_{x_{1}}+\beta_{2} r_{x_{2}}+\beta_{3} r_{x_{3}}
$$

where $r_{y}$ represents the ranks of the observations on $Y, r_{X}$ represents the ranks of the observations on $X_{1}$, and so on, just as in the simpler examples given earlier. The ranks for the data in Figure 13 are given in Figure 14. The least squares method is used to find the values of the coefficients $B_{0}$ through $\beta_{3}$, which appear as part of the computer output. For the data in Figure 12 the coefficients are

$$
r_{y}=-0.31+.6650 r_{x_{1}}+.3859 r_{x_{2}}-.0226 r_{x_{3}}
$$

The predicted rank for each observation is obtained by substituting the respective ranks of the independent variables into Equation (9). As an example the ranks of $X_{1}, X_{2}$ and $x_{3}$ for observation number 1 are given in Figure 14 as $r_{X_{1}}=20.5, r_{X_{2}}=20.5$ and $r_{X_{3}}=15$ respectively. These are substituted into Equation (9) to get

$$
\begin{aligned}
\hat{r}_{y} & =-0.31+(.6650)(20.5)+(.3859)(20.5)-(.0226)(15) \\
& =20.89
\end{aligned}
$$

in agreement with the number given in Figure 14 for observation number 1. To convert this predicted rank, $\hat{r}_{y}=20.89$, to a predicted value for $Y$ the two values of $Y$ : whose ranks straddle 20.89 are found from Figure 14. These are $Y=42$, whose rank is 21 , and $Y=37$, whose rank is 19.5. Interpolation gives the predicted value of $Y$,

$$
\begin{aligned}
\hat{Y} & =37+\frac{20.89-19.5}{21-19.5}(42-37) \\
& =41.63
\end{aligned}
$$

which agrees with the value from Figure 13 , except for differences caused by using rounded-off values of the coefficients in Equation (9).

\section{Exercise 3}

Obtain the predicted rank in observation number 4 by substituting the ranks from Figure 14 into Equation (9). See if this agrees with the predicted rank given in Figure 14 . 
Exercise 4

Use the predicted rank $\hat{r}_{y}$ for observation number 4 from Figure 14 to obtain a predicted value for $Y$. See if this agrees with the number given in Figure 13.

\section{Comparing Ordinary Regression and Rank Regression}

A measure of the goodness of $f i t$ of the rank regression model is obtained by comparing each predicted value $\hat{Y}$ with the corresponding observed value $Y$. The sum of squares of the residuals is

$$
\begin{aligned}
S S & =\Sigma(Y-\hat{Y})^{2} \\
& =142.63
\end{aligned}
$$

which is less than the value 178.83 obtained using ordinary regression. Although ordinary regression finds the best fitting hyperplane, rank regression is not restricted to working with a single hyperplane. Actually a series of connected hyperplanes is obtained using rank regression, so the model can adjust to the data with some degree of flexibility, within the constraint of being monotone in each of its variables. Any disagreement between the residuals in $F$ igure 13 and the residuals reported in Iman and Conover (1979) is due to a difference in the method of handling ties.

Sensitivity Analysis

It is apparent from the relative size of the coefficients in Equations (7) and (9) that the output is more sensitive to changes in some independent variables than to changes in others. For example, $X_{1}$ ranges from 50 to 80 , a change of 30 units. The coefficient of $x_{1}$ in Equation (7) is .7156 , so the maximum change in $Y$ due to changes in $X_{1}$ is $(30)(.7156)=21.5$ units. On the other hand $x_{3}$ ranges from 72 to 93 , a distance of 21 units, and has a coefficient of -..1521 in Equation (7). The maximum change in $Y$ due to changes in $x_{3}$ is only 3.2 units, less than one-sixth of the total influence of $x_{1}$. The situation is further complicated by the fact that $x_{1}$ and $x_{3}$ have a positive correlation coefficient of $r_{13}=.500$. This suggests that if $x_{3}$ were dropped from the regression model, the coefficient of $x_{1}$ might increase somewhat to account for some of the variability in $Y$ that was formerly accounted for by $x_{3}$. Thus $x_{3}$ might not be making a significant contribution in the model, and perhaps should be omitted. Statistical tests are available for aiding in the decision of whether or not to omit variables from the model.

A similar line of reasoning may be used on the least squares fit to the ranks, only here the analysis is simpler because all of the ranks have approximately the same range. Only the coefficients in Equation (9) need to be examined. The coefficient of $r_{x_{3}}-.0226$ is about one-thirtieth the size of the coefficient of $r_{X_{1}}, .6650$, aga in suggesting that the rank of $Y$ is much less sensitive to changes in the rank of $X_{3}$ than to changes in the rank of $x_{1}$. 
Obs.

\begin{tabular}{|c|c|c|c|c|c|c|c|c|c|}
\hline No. & $Y$ & $r_{y}$ & $\hat{r}_{y}$ & $x_{1}$ & $r_{x_{1}}$ & $x_{2}$ & $r_{x_{2}}$ & $x_{3}$ & $r_{x_{3}}$ \\
\hline 1 & 42 & 21 & 20.89 & 80 & 20.5 & 27 & 20.5 & 89 & 15 \\
\hline 2 & 37 & 19.5 & 20.95 & 80 & 20.5 & 27 & 20.5 & 88 & 12.5 \\
\hline 3 & 37 & 19.5 & 19.27 & 75 & 19 & 25. & 19 & 90 & 17 \\
\hline 4 & 28 & 18 & 16.01 & 62 & 15 & 24 & 17 & 87 & 9.5 \\
\hline 5 & 18 & 14.5 & 14.47 & 62 & 15 & 22 & 13 & 87 & 9.5 \\
\hline 6 & 18 & 14.5 & 15.04 & 62 & 15 & 23 & 14.5 & 87 & 9.5 \\
\hline 7 & 19 & 16 & 15.77 & 62 & 15 & 24 & 17 & 93 & 20 \\
\hline 8 & 20 & 17 & 15.77 & 62 & 15 & 24 & 17 & 93 & 20 \\
\hline 9 & 15 & 12 & 11.39 & 58 & 9.5 & 23 & 14.5 & 87 & 9.5 \\
\hline 10 & 14 & 9.5 & 7.47 & 58 & 9.5 & 18 & 4 & 80 & 3.5 \\
\hline 11 & 14 & 9.5 & 7.21 & 58 & 9.5 & 18 & 4 & 89 & 15 \\
\hline 12 & 13 & 8 & 6.11 & 58 & 9.5 & 17 & 1 & 88 & 12.5 \\
\hline 13 & 11 & 6 & 7.43 & 58 & 9.5 & 18 & 4 & 82 & 5.5 \\
\hline 14 & 12 & 7 & 8.64 & 58 & 9.5 & 19 & 8 & 83 & 20 \\
\hline 15 & 8 & 3 & 2.89 & 50 & 3 & 18 & 4 & 89 & 15 \\
\hline 16 & 7 & 1 & 3.07 & 50 & 3 & 18 & 4 & 86 & 7 \\
\hline 17 & 8 & 3 & $4.7,5$ & 50 & 3 & 19 & 8 & 72 & 1 \\
\hline 18 & 8 & 3 & 4.73 & 50 & 3 & 19 & 8 & 79 & 2 \\
\hline 19 & 9 & 5 & 5.85 & 50 & 3 & 20 & 11 & 80 & 3.5 \\
\hline 20 & 15 & 12 & $7.80^{\circ}$ & 56 & 6 & 20 & 11 & 82 & 5.5 \\
\hline 21 & 15 & 12 & 15.50 & 70 & 18 & 20 & 11 & 91 & 18 \\
\hline
\end{tabular}

Figure 14. The Ranks of the Data in Figure 13 and the Predicted Ranks from Equation (9). 
On Deciding What Variables to Include in the Model

The inclusion of many variables in the regression model may result in "overfitting" the data. That is, the effect of having many variables is to force the regression surface into wildly erratic patterns just so it will pass closer to the observed points and have smaller residuals. Some systematic method is needed to assist in deciding whether variables should be included or excluded from the analysis. With such a tool to aid in the decision making, one may consider other variables related to $x_{1}, x_{2}$ and $x_{3}$, such as $x_{1}^{2}$ or $x_{1} x_{2}$, because it is possible that these other variables are more useful than the simple ones that have been considered so far. Three of these decision-assisting tools will be introduced and compared. But first the concept of partial correlation needs to be explained.

\section{Simple Correlation}

The strength of a simple linear relationship between two variables in usually measured with $r$, called Pearson's product moment correlation coefficient, or simply, the correlation coefficient for short. The correlation coefficient between $X$ and $Y$ is given by

$$
r=\frac{\sum\left(X_{i}-\bar{X}\right)\left(Y_{i}-\bar{Y}\right)}{\sqrt{\Sigma\left(X_{i}-\bar{X}\right)^{2} \Sigma\left(Y_{i}-\bar{Y}\right)^{2}}}
$$

for paired observations observations $\left(X_{1}, Y_{1}\right), \ldots,\left(X_{n}, Y_{n}\right)$, where $\bar{X}$ and $\bar{Y}$ are the sample means. The statistic $r$ may be used to test the hypothes is of no correlation, but only if the variables have a particular distribution called bivariate normal distribution. This condition is often assumed, but rarely met, in practice.

When more than two variables are involved subscripts are used to show which two variables are being correlated. Thus $r_{12}$ refers to the correlation between $x_{1}$ and $x_{2}$,

$$
r_{12}=\frac{\Sigma\left(x_{1 i}-\bar{x}_{1}\right)\left(x_{2 i}-\bar{x}_{2}\right)}{\sqrt{\Sigma\left(x_{1 i}-\bar{x}_{1}\right)^{2} \Sigma\left(x_{2 i}-\bar{x}_{2}\right)^{2}}}
$$

while $r_{y 1}$ refers to the correlation between $Y$ and $X_{1}$.

\section{Rank Correlation}

The strength of a monotonic relationship between two variables, as opposed to a linear relationship, is usually measured using $r$, but computed on the ranks of the variables instead of the variables themselves. It is customary to use either $r_{S}$ or $\rho$ (rho) to denote the rank correlation coeffi- 
cient, sometimes called Spearman's rho. We will try to minimize problems with subscripts by using $\rho$, such as. 12

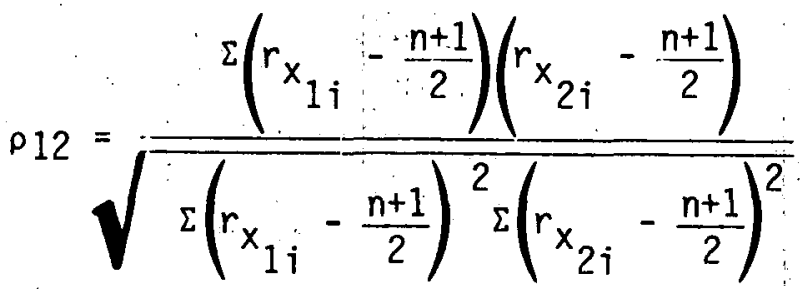

to denote the correlation between the ranks $r_{X}$; of $X_{1}$ and the ranks $r_{X}$ of $x_{2}$. All of the calculations and interpretation of results using the correlation on the data may be applied to the ranks of the data as well. The regression program described by Iman, et al (1980) handles all of the calculations on the data or the ranks of the data, the calculations just described and the calculations in the following pages. Spearman's rho may be used to test the hypothesis of independence, without requiring any distributional assumptions. Special tables may be found in Conover (1980).

\section{Partial Correlation}

Sometimes the apparent correlation between two variables may be due in part to the indirect influence of a third variable on both of the other variables. For example, the weekly average municipal bond yield $x_{1}$ may appear to be correlated with the average utility bond yield $x_{2}$ for the same week. Yet both may be heavily influenced by the average interest rate charged by the Federal Reserve, $x_{3}$, for that week. How can the influence of the variable $X_{3}$ be removed from the relationship between $X_{1}$ and $X_{2}$ ?

One way to do this is to use a simple linear regression of each variable $X_{1}$ and $X_{2}$ separately on $X_{3}$. The least squares method is used to fit coefficients to

$$
\hat{x}_{1}=\beta_{0}+\beta_{1} X_{3}
$$

to get $t_{A}$ the residuals $\left(x_{1}-\hat{x}_{1}\right)$. In a similar manner the residuals $\left(x_{2}-x_{2}\right)$ are al so obtained by a regression of $x_{2}$ on $x_{3}$. Thus the 1 inear influence of $x_{3}$ on both $x_{1}$ and $x_{2}$ is removed, and the correlation between the residuals $\left(x_{1}-X_{1}\right)$ and $\left(x_{2}-x_{2}\right)$ can be computed using Equation $(10)$, but where $r$ is computed using $\left(x_{1}-x_{1}\right)$ instead of $x_{1}$, and $\left(x_{2}-\hat{x}_{2}\right)$ instead of $x_{2}$.

\section{An Equation for Computing Partial Correlation}

Such a correlation coefficient is called the partial correlation coefficient between $x_{1}$ and $x_{2}$, given $x_{3}$ and is denoted by $r_{12.3}$. An easy way to compute $r_{12} .3$ is with the equation

$$
r_{12.3}=\frac{r_{12}-r_{13} r_{23}}{\sqrt{\left(1-r_{13}^{2}\right)\left(1-r_{23}^{2}\right)}}
$$


where $r_{12}, r_{13}$ and $r_{23}$ are the simple linear correlatior coefficients

between $X_{1}$ and $X_{2}, X_{1}$ and $X_{3}$, and $X_{2}$ and $X_{3}$ respectively. The relationship given by Equation (13) obtains the same partial correlation coefficient as would be obtained by going through the process of correlating residuals described earlier.

\section{Partial Rank Correlation}

The use of rank regression, and the rank correlation coefficients $\rho 12$, $\rho_{13}$, and $\rho_{23}$ gives $\rho_{12.3}$, which measures the strength of the 1 inear association between the ranks of $X_{1}$ and the ranks of $X_{2}$, after the linear effect of the ranks of $x_{3}$ is removed from the ranks of $x_{1}$, and the ranks of $x_{2}$. The equation

$$
\rho_{12.3}=\frac{\rho_{12}-\rho_{13} \rho_{23}}{\sqrt{\left(1-\rho_{13^{2}}\right)\left(1-\rho_{23^{2}}\right)}}
$$

may be used to find the partial rank correlation coefficient.

\section{Partial Correlation Given Several Variables}

The concept of partial correlation is easily extended to measure the strength of the linear relationship between two variables $x_{1}$ and $x_{2}$, after the linear effects of several variables, say $x_{3}, x_{4}$ and $x_{5}$, have been removed. One way of looking at this partial correlation is to compute the correlation on the residuals $\left(x_{1}-\hat{x}_{1}\right)$ and $\left(x_{2}-\hat{x}_{2}\right)$ as before, but where $x_{1}$ and $x_{2}$ are obtained from a 1 inear regression on $x_{3}, x_{4}$, and $x_{5}$. That is, the model

$$
\hat{X}_{1}=\beta_{0}+\beta_{1} X_{3}+\beta_{2} X_{4}+\beta_{3} X_{5}
$$

is fitted using least squares, and so is the model

$$
\hat{x}_{2}=\beta_{0}^{\prime}+\beta^{-}{ }_{1} X_{3}+\beta^{\prime}{ }_{2} X_{4}+\beta^{\prime}{ }_{3} X_{5}
$$

in order to remove the unwanted influence of $x_{32} x_{4}$ and $x_{5}$ on the variables $x_{1}$ and $x_{2}$. The correlation between $\left(x_{1}-x_{1}\right)$ and $\left(x_{2}-\hat{x}_{2}\right)$ is

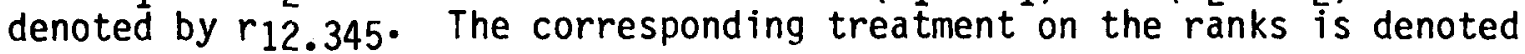
by $\rho 12.345$

Another way of finding $r_{12.345}$ is by the equation 


$$
r_{12.345}=\frac{r_{12.45-r_{13.45} r_{23.45}}}{\sqrt{\left(1-r_{13.45}\right)\left(1-r_{23.45}\right)}}
$$

which is analogous to Equation (13).

\section{Three Multiple Regression Procedures}

Correlation coefficients and partial correlation coefficients are used in several different ways to assist in deciding which variables to include in a regression model involving $Y$ and several independent variables. Three such methods will be described.

1. The forward procedure. The regression model starts with no variables in it. Variables, are then added, one at a time, to the regression model until a decision is reached that no new variables will contribute significantily to the improvement of the fit of the model to the data.

2. The backward procedure. The regression model starts with all of the variables in it. Then variables which are considered not making a significant contribution to the fit are dropped one by one until all insignificant variables have been removed from the model.

3. The stepwise procedure. The regression model starts with no variables in it. Variables are then added, one at a time, as in the forward procedure.: Each time a variable is added, the backward procedure is used to delete all variables previously added to the model but which are now considered to be insignificant in the presence of the new variable. This combination of the forward and backward procedures is the most commonly used method of determining which set of variables belongs in the model.

\section{The Variables Being Considered}

These methods for assisting in deciding which variables to include enable many more variables to be considered than one would normally use in a model. - In the backward procedure the number of variables must be less than the number of data points being fitted, but in the forward and stepwise procedures no such limitation exists. For the example introduced earlier, the original yariables $x_{1}, x_{2}$ and $x_{3}$ will be considered, plus the squared terms $x_{1}^{2}, x_{2}^{2}, x_{3}^{2}$, and the cross products $x_{1} x_{2}, x_{1} x_{3}$, and $x_{2} x_{3}$. Thus nine independent variables will be considered in this regression model. Many other variables could be included, such as $1 / X_{1}, X_{1} / X_{2}, \sqrt{X_{3}}, \ln X_{2}$, and so on. Any variables which may have a real physical interpretation in the situation being modeled should be considered. In our example we will consider only the nine first and second order terms, as mentioned. For convenience the variables, including $Y$, are numbered from 1 to 10 , as shown in Figure 15. 


\section{The Forward Procedure}

The forward procedure starts with a model that has no variables. The first variable chosen to be included in the model is the one that has the largest correlation with $Y$. The largest value of $r$ for the data in Figure 12 and involving $Y$ as one of the variables is the correlation coefficient between $Y$ and $X_{1} X_{2}$

$$
r_{18}=.9588
$$

so $x_{1} x_{2}$ is the first variable considered for inclusion in the model.

\section{A Test of Significance}

At this point a test of significance is conducted to see if the inclusion of $X_{1} X_{2}$ in the model reduces the unexplained variation of $Y$ a significant amount. The statistical test used is exact only if several assumptions regarding the distribution of $Y$ are true. These assumptions are well. explained in textbooks which present regression methods (see Draper and Smith, 1966), so they are not repeated here. In practice these assumptions are, at best, only approximately true, so this test and all subsequent statistical tests are only approximate. Still they serve as a useful objective method for assisting in making the decision as to whether or not a variable should be included. The test indicates (at $\alpha=.05$ ) that $x_{1} x_{2}$ should be included, so the forward procedure continues.

\section{Using Partial Correlation in Forward Regression}

The partial correlation coefficients of each variable with $Y$, given $X_{1} X_{2}$, are compared and the largest one is selected. Partial correlations are computed using Equation (13) in conjunction with the correlations given in Figure 15 .

For example, the partial correlation of $Y$ with $x_{1}^{2}$, given $x_{1} x_{2}$ is

$$
\begin{aligned}
r_{15.8} & =\frac{r_{15}-r_{18} r_{58}}{\sqrt{\left(1-r_{18}\right)\left(1-r_{58}\right)}} \\
& =\frac{.9251-(.9588)(.9500)}{\sqrt{\left(1-(.9588)^{2}\right)\left(1-(.9500)^{2}\right)}} \\
& =.1605
\end{aligned}
$$

Exercise 5

Use Equation 13 and the correlation "coefficients given in Figure 15 to find the partial correlation coefficient of $Y$ and $X_{1}$, given $X_{1} X_{2}$. 


\begin{tabular}{|c|c|c|c|c|c|}
\hline $\begin{array}{l}\text { Variable } \\
\text { Number }\end{array}$ & $\begin{array}{l}\text { Variable } \\
\text { Name }\end{array}$ & $\begin{array}{l}\text { Correlation } \\
\text { With } Y\end{array}$ & $\begin{array}{c}\text { Correlation } \\
\text { With } x_{1} X_{2}\end{array}$ & $\begin{array}{c}\text { Rank } \\
\text { Correlation } \\
\text { With } Y\end{array}$ & $\begin{array}{c}\text { Rank } \\
\text { Correlation } \\
\text { With } x_{1} x_{2}\end{array}$ \\
\hline 1 & Y & 1.0000 & 9588 & 1.0000 & .9224 \\
\hline 2 & $x_{1}$ & 91197 & .9453 & .9180 & . 9099 \\
\hline 3 & $x_{2}$ & .8755 & .9378 & .8521 & .9188 \\
\hline 4 & $x_{3}$ & .3998 & .4508 & .4974 & .4985 \\
\hline 5 & $x_{1}^{2}$ & .9251 & .9500 & .8896 & .9519 \\
\hline 6 & $x_{2}^{2}$ & .8934 & .9499 & .8897 & .9628 \\
\hline 7 & $x_{3}^{2}$ & .3958 & .4497 & .4161 & .4352 \\
\hline 8 & $x_{1} x_{2}$ & .9588 & 1.0000 & .9224 & 1.0000 \\
\hline 9 & $x_{1} x_{3}$ & .8712 & .9094 & .7892 & .8249 \\
\hline 10 & $x_{2} x_{3}$ & .8554 & .9239 & .8276 & .8862 \\
\hline
\end{tabular}

Figure 15. Some Correlation Coefficients for the Data from Figure 12 and the Ranks from Figure 14.

Adding Another Variable to the Model

The largest partial correlation coefficient is found, and that variable is examined using the test of significance, to see if including it in the model reduces the variation of $Y$ a significant amount. In this case the test shows nonsignificance, so no additional variables are included in the model.

The Forward Regression Model

Only the variable $x_{1} x_{2}$ is included in the model, of the nine variables examined, for the data in Figure 12. The least squares fit results in the model

$$
\hat{\gamma}=-15.29+.025315 x_{1} x_{2}
$$

The $R^{2}$ value is .919 for this model, indicating that $91.9 \%$ of the variation in $Y$ is accounted for by a 1 inear regression on $X_{1} X_{2}$. This is about the same as the $R^{2}=.914$ obtained for the model in Equation (7) which used the variables $X_{1}, X_{2}$ and $x_{3}$. The inclusion of additional variables automatically increases the value of $R^{2}$, generally, a model with on ly gne variable is preferred over a model with the same value of $R^{2}$ but with three variables. 


\section{Obtaining Predicted Values in Multiple Regression}

To obtain a predicted value from Equation (16) the value of $x_{1}$ is multiplied by $x_{2}$, and the product is placed in the equation. For example, in observation number 1 in Figure 12, $x_{1}=80$ and $x_{2}=27$. Then Equation (16) becomes

$$
\begin{aligned}
\hat{Y} & =-15.29+.025315 x_{1} x_{2} \\
& =-15.29+(.025315)(80)(27) \\
& =39.39
\end{aligned}
$$

in agreement with the predicted value given in Figure 16. The sum of squares of residuals for this model is

$$
S S=116.846
$$

which is considerably better (smaller) than the value 178.83 given in Figure 13 for the model with variables $X_{1}, X_{2}$ and $X_{3}$. This forward regression method has found a better model, with fewer variables, than the model examined earlier.

\section{The Forward Rank Regression Procedure}

Forward rank regression proceeds just as described for ordinary forward regression, except $x_{1}, x_{2}$ and $x_{3}$ are replaced by their ranks initially. Then terms corresponding to $x_{1}{ }^{2}$ or $x_{1} x_{2}$ become the square of the rank of $x_{1}$, or the rank of $x_{1}$ times the rank of $x_{2}$.

The largest simple rank correlation is

$$
\rho_{18}=.9224
$$

so the rank analogue to $x_{1} x_{2}$ is selected for possible inclusion in the model. The test of significance indicates, at $\alpha=.05$, that this variable should be included in the model.

The largest partial correlation with the rank of $Y$, given the rank analogue of $X_{1} X_{2}$, involves the rank of the variable $X_{1}$ 
Least Squares

Obs. Observed

No. Value $Y$

1

2

3

4

5

6

7

8

9

10

11

12

13

14

15

16

17

18

19

20

21
Model: $x_{1} x_{2}$

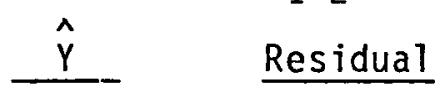

39.39

39.39

32.17

22.38

19.24

20.81

22.33

22.38

18.43

11.14

11.14

9.67

11.14

12.60

$7.49 !$

7.49

8.76

8.76

10.02

13.06

20.15

2.61

$-2.39$

4.83

5.62

$-1.24$

$-2.81$

$-3.38$

$-2.38$

$-3.48$

2.86

2.86

3.33

$-0.14$

$-0.60$
0.51

$-0.49$

$-0.76$

$-0.76$

$-1.02$

0.51
-0.49
-0.76
-0.76
-1.02
1.94
-5.15

$S S=116.846$
15

15

9
Rank Regression

Model: $x_{1}, x_{2}^{2}, x_{1} x_{2}$

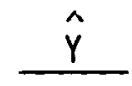

Residual

40.40

1.60

40.40

$-3.40$

33.95

3.05

19.80

8.20

16.59

1.41

18.00

0.00

19.80

$-0.80$

19.80

0.20

16.37

$-1.37$

12.70

1.30

12.70

1.30

13.44

$-0.44$

12.70

$-1.70$

13.10

$-1.10$

7.34

0.66

7.34

$-0.34$

8.00

0.00

8.00

0.00

11.81

$-2.81$

13.13

1.87

17.06

$-2.06$

$S S=119.176$

Figure 16. Predicted Values and Residuals from the Forward Regression Models on the Data and on the Ranks. 


$$
\begin{aligned}
\rho_{12.8} & =\frac{\rho_{12-\rho_{18} \rho_{28}}}{\sqrt{\left(1-\rho_{18}^{2}\right)\left(1-\rho_{28}^{2}\right)}} \\
& =\frac{.9180-(.9224)(.9099)}{\sqrt{\left(1-(.9224)^{2}\right)\left(1-(.9099)^{2}\right)}} \\
& =.4912
\end{aligned}
$$

and this is large enough to be declared significant, using the same statistical test that is used for $r_{12.8}$. Therefore the variable $X_{1}$ is added to the model.

The next step consists of examining all partial correlations with the rank of $Y$, given the rank analogues to $X_{1}$ and $X_{1} X_{2}$. The largest partial rank correlation coefficient belongs to the rank analogue of $x_{2}{ }^{2}$, and that term is included in the model after a statistical test determines its significance. No more terms are found to be significant. The least squares method is used to obtain the coefficients, with the result

$$
\hat{r}_{y}=-2.11+1.1938 r_{x_{1}}+.06227 r_{x_{2}}^{2}-.06666 r_{x_{1}} r_{x_{2}}
$$

\section{Using Rank Regression to Predict Values of $Y$}

To find a predicted rank for $Y$, the corresponding ranks for $X_{1}$ and $X_{2}$ are substituted into Equation (17). For example, in observation number 1 of Figure 14 the ranks are $r_{x_{1}}=20.5$ and $r_{x_{2}}=20.5$. This gives

$$
\begin{aligned}
\hat{r}_{y} & =-2.11+1.1938(20.5)+.06227(20.5)^{2}-.06666(20.5)(20.5) \\
& =20.52
\end{aligned}
$$

The two ranks which straddle the value 20.52 are, from Figure 14, 21 $(Y=42)$ and $19.5(Y=37)$. Interpolation gives

$$
\begin{aligned}
\hat{y} & =37+\frac{20.52-19.5}{21-19.5}(42-37) \\
& =40.40
\end{aligned}
$$

which is in agreement with the value given in Figure 16. 
Use the ranks of $X_{1}$ and $X_{2}$ for observation number 9 in Figure 14 to find $\hat{r}_{y}$ from Equation 17 . (The computer printout gives $\hat{r}_{y}=13.14$. )

Exercise 7

Use interpolation in Figure 14 to obtain a predicted value for $Y$, given $\hat{r}_{y}=13.14$ for observation number 9 , and see if your answer agrees with the predicted value given in Figure 16 .

Comparing Rank Regression with Ordinary Regression

The sum of squares of residuals for the rank regression model given by Equation (17) is

$$
\mathrm{SS}=119.176
$$

which is about the same as the value found using forward regression on the data. Of the two models found using forward regression, the model based on the data is preferred, because it has about the same value for SS as the model based on ranks, and it has only one variable as opposed to three variables for the model based on ranks.

\section{Backward Regression}

The backward regression procedure begins with the model containing all of the variables, fitted using the least squares method. Then the partial correlation coefficients of each variable with $Y$, given all of the other variables in the model, are compared to see which one is the smallest. The variable corresponding to this smallest partial rank correlation coefficient is then tested to see if its presence in the model contributes significantly in accounting for the variation in $Y$. If the test is significant the variable remains in the model and the procedure is finished. If the variable does not test as significant, it is dropped from the model, and the entire procedure is repeated for the remaining variables. At each stage the partial correlations involve only the variables remaining in the model. This backward regression procedure has a tendency to include more variables in the final model than if forward regression is used.

\section{A Useful Procedure for Finding Partial Correlation Coefficients}

At this point a very useful technique will be introduced and illustrated. The method for finding partial correlation coefficients by building step by step from the simple correlation coefficients, as described earlier, works well for forward regression. This is an awkward way of handling backward regression however, since backward regression starts with the full model, all variables included, and requires knowledge of the partial correlation coefficients given all but one of the independent variables. There is a very simple way to do this.

Let $R$ be the correlation matrix; that is, the matrix containing the simple pairwise correlation coefficients where row $i$, column $j$ contains $r_{i j}$. 
Let $R^{-1}$ be the inverse matrix of $R$, which is the matrix that can be multiplied by $R$ to get the identity matrix 1 . Many software packages exist for finding $R^{-1}$. Denote the element in row $i$, column $j$ of $R^{-1}$ by $b_{i j}$. The partial correlation of variable $i$ with variable $j$, given all of the other variables represented in the correlation matrix, is obtained very simply from $R^{-1}$ as

$$
r_{i j \cdot(a) \text { others })}=-\frac{b_{i j}}{\sqrt{b_{i j} b_{j j}}}
$$

When a variable is el iminated from the model because it is not significant, the row and column in $R$, which represent correlations involving that variable, are removed from $R$ to obtain a new and smaller correlation matrix $R_{1}$. The inverse $R_{1}{ }^{-1}$ of $R_{1}$ is found and the new partial correlation coefficients of each variable, with $Y$, given the variables still remaining in the model, are found from $R_{1}^{-1}$ using Equation (18).

An Illustration of the Procedure

This procedure for finding partial correlation coefficients could be illustrated using the full model, with $Y$ and nine independent variables. This would involve a correlation matrix $R$ with 10 rows and 10 columns, and

its inverse $R^{-1}$ al so with 10 rows and 10 columns. Such an example is so large that it may confuse the illustration of the procedure, so the procedure will be illustrated using only $Y$ and the original independent variables $x_{1}, x_{2}$ and $x_{3}$.

For the data in Figure 12 the correlation matrix is given by

$$
R=\left[\begin{array}{rrrr}
1.0000 & X_{1} & X_{2} & Y \\
.7819 & 1.7819 & .5001 & .9197 \\
.5001 & .3909 & .3909 & .8755 \\
.9197 & .8755 & .3900 & .3998 \\
& .3900000
\end{array}\right]
$$

where row 4 (and column 4 ) contains the correlation coefficients of $Y$ with $x_{1}, x_{2}$ and $x_{3}$ and itself, respectively.

The inverse matrix is found from a computer program as

$$
R^{-1}=\left[\begin{array}{rccc}
X_{1} & \multicolumn{1}{c}{X_{2}} & \multicolumn{1}{c}{X_{3}} & y \\
.7241 & .9922 & -1.2655 & -7.4665 \\
-1.2922 & 4.4469 & -.3727 & -4.6568 \\
-7.4665 & -4.6727 & 1.4078 & .9274 \\
-968 & .9274 & 11.5732
\end{array}\right]
$$

Note that both $R$ and $R-1$ are symmetric matrices. 
The partial correlation coefficients are found using Equation (18). Only the partial correlations involving $Y$ are of interest, since these represent the amount of influence each independent vartable has on $Y$, when the linear influence of the other variables is removed. These are

$$
\begin{aligned}
& Y \text { and } x_{1}: \quad r_{41.23}=\frac{-b_{41}}{\sqrt{b_{44} b_{11}}}=\frac{7.4665}{\sqrt{(7.7241)(11.5732)}}=.7897 \\
& Y \text { and } X_{2}: \quad r_{42.13}=\frac{-b_{42}}{\sqrt{b_{44} b_{22}}}=\frac{4.6568}{\sqrt{(4.4469)(11.5732)}}=.6491 \\
& Y \text { and } X_{3}: \quad r_{43.12}=\frac{-b_{43}}{\sqrt{b_{44} \cdot b_{33}}}=\frac{-.9274}{\sqrt{(1.4078)(11.5732)}}=-.2298
\end{aligned}
$$

In a backward regression procedure the variable $x_{3}$ would be selected for testing because it has the lowest partial correlation coefficient (in absolute value). If the test showed that $x_{3}$ 's contribution to the model were insignificant it would be dropped from the model. Row 3 and column 3 would be removed from the correlation matrix $R$, the new inverse matrix found, and the new partial correlation coefficients found with $x_{3}$ eliminated from all further consideration. This procedure would be repeated until all varibles remaining in the model are significant.

Measuring the Importance of Variables

A direct interpretation of the partial correliation coefficients found from the full model is very useful. The three variables $x_{1}, x_{2}$ and $X_{3}$ may be ranked in order of importance on the basis of their partial correlation coefficients. Variable $x_{1}$ is the most important because its partial correlation coefficient, $r_{41.23}=.7897$, is the largest in absolute value. The variable $X_{2}$ is the second most important variable and $x_{3}$ is the least important variable. Although a rough analys is was made when this model was introduced in Equation (7), and resulted in these same conclusions, this method of analysis removes from consideration the indirect influence of the other variables, and thus isolates the effect of each variable on $Y$ in a more precise manner.

\section{The Results Using Backward Regression}

The backward regression procedure on all nine independent variables removed the variable $x_{2}{ }^{2}$ in its first step. Subsequent steps removed, one by one, the variables $x_{2} x_{3}, x_{1}, x_{3}, x_{1} x_{3}, x_{3}, x_{1}$ and $x_{2}$, leaving $x_{1} x_{2}$ as the only significant variable. Thus the backward regression procedure ended up with the same model as was obtained from the forward regression procedure. This is merely a coincidence that happened to occur in this case. The predicted values, the residuals, and the residual sum of squares are thus given in Figure 16. This lends further support to the conclusion that the model involving only $x_{1} x_{2}$ is a good model for the data.

$$
-97-
$$


Backward Rank Regression

Backward regression on the rank's follows the same steps as backward regression on the data. The only difference is that the ranks of $Y, X_{1}, X_{2}$ and $X_{3}$ are substituted for the actual values of those variables. The partial rank correlations are found by inverting the matrix of simple rank correlations and using

$$
\rho_{i j .(a l l ~ o t h e r s)}=\frac{-b_{i j}}{\sqrt{b_{i j} b_{j j}}}
$$

in a manner entirely analogous to the procedure described previously.

The backward regression procedure on the ranks given in Figure 14 results in the el imination of variable $x_{2}$ on the first step. Note that the analysis on the actual data differed in this respect, because $x_{2}{ }^{2}$ was removed on the first step, and the variable $x_{2}$ was the last one to be removed.

Bear in mind that regression on ranks is based on monotonic relationships, while regression on the data is based on linear relationships, so removal of $x_{2}$ using rank regression is not entirely different than the removal of $x_{2}{ }^{2}$ using ordinary regression.

The Model from Backward Rank Regression

Subsequent steps in the backward rank regression progedure removed, one at a time, the variables $x_{1} x_{3}, x_{2} x_{3}, x_{1} x_{2}, x_{3}$, and $x_{3}{ }^{2}$. This left the variables $x_{1}, x_{1}^{2}$ and $x_{2}$ in the model. Several interesting things happened. The variable $x_{1}^{2} x_{2}$ was removed on the fourth step. This is one of the three variables which remained in the model using forward regression on the ranks, and the only variable which remained in the model using both forward regression and backward regression on the actual data. Also, the variable $x_{1} 2$ remains in the model for backward regression on the ranks. This is the first time this variable has appeared in a regression model. It is interesting to see what effect the replacement of $x_{1} x_{2}$ with $x_{1}{ }^{2}$ has on the rank regression model, as this represents the only difference between the forward regression model on the ranks and the backward regression model on the ranks. The final model is given as

$$
\hat{r}_{y}=-2.02+1.41 r_{x_{1}}-.04410 r_{x_{1}}^{2}+.02781 r^{2} x_{2}
$$

The predicted values of $Y$ and the residuals appear in Figure 17.

\section{A Comparison of the Several Models}

The model given by Equation (20) appears to be the best model obtained so far, as measured by the sizes of the residuals given in Figure 
17. When compared with forward rank regression, the backward rank regression model resulted in smaller residuals for 10 of the 21 points, larger residuals for 9 of the 21 points, and ties in 2 cases. However, the important measure of goodness of fit, the sum of squares of residuals, is reduced by about 23 percent, to

$$
\text { SS }=91.677
$$

which is by far the smallest value for SS yet obtained. This is smaller than the value 116.846 obtained using the model using $X_{1} X_{2}$ on the actual data. One model has a smaller SS, while the other model has fewer variables. It is difficult to choose between these two models at this point.

StepwiserRegression

The stepwise regression procedure is the procedure that is used most, often in obtaining a regression model. It begins as a forward regression procedure, but each time a variable is added to the model it becomes a backward regression procedure until all insignificant variables have been el iminated from the model. When a new variable is added to the model, the partial correlation coefficients consider this new variable in addition to the previous variables, and they may be different than the previous partial correlation coefficients. This is why ariable that was previously considered significant using forward regression may become insignificant after a new variable is added, in which case it would be dropped using backward regression. This does not preclude that variable from being added again at some later point, if it aga in becomes significant after additional variables have entered the model.

The application of the stepwise regression procedure to the data - in $F$ igure 12 and the ranks in $F$ igure 14 is not interesting, because in those cases no variables are eliminated from the model as insignificant. Therefore the resulting models, predicted values, and residual;s are the same as those obtained using forward regression, and given in Figure 16.

\section{Discussion}

Several regression procedures have been introduced and described for constructing a regression model involving several variables. Partial correlation is discussed as a tool for identifying important variabies. The computations have been expla ined so that a better understanding of the powers and limitations of the various procedures can be obtained.

All of these procedures are merely aids in the decision making process. They should be considered in addition to expert advice, not instead of expert advice. Amateur statisticians of ten make the mistake of having either too little or too much fath in the methods of regression analys is. Professional statisticians of ten make the same mistake, but to a lesser extent. The better these regression methods are understood, the more likely $i$ it is that the results of a regression analys is will be given its proper weight in the final decision making process. 
Forward Rank Regression: $x_{1}, x_{2}^{2}, x_{1} x_{2}$

Obs.

No.

1

2

3

4

5

6

7

8

9

10

11

12

13

14

15

16

17

18

19

20

21
Observed

Value Y

42

37

37

28

18

18

19

20

15

14

14

13

11

12

8

7

8

8

9

15

15

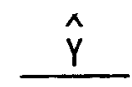

40.40

40.40

33.95

19.80

16.59

18.00

19.80

19.80

.16 .37

12.70

12.70

13.44

13.44

13.10

7.34

7.34

8.00

8.00

11.81

13.13

17.06
Residual

1.60

$-3.40$

3.05

8.20

1.41

0.00

$-0.80$

0.20

$-1.37$

1.30

1.30

$-0.44$

$-1.70$

$-1.10$

0.66

$-0.34$

0.00

0.00

$-2.81$

1.87

$-2.06$

$S S=119.176$
Backward Rank

Regression: $x_{1}, x_{1}^{2}, x_{2}^{2}$.

Residual

38.92

3.08

38.92

$-1.92$

33.54

3.46

22.18

5.82

17.32

0.68

18.39

$-0.39$

22.18

$-3.18$

22.18

$-2.18$

16.51

$-1.51$

12.86

1.14

1.14

12.86

0.56

12.44

$-1.86$

12.86

$-2.00$

14.00

0.00

8.00

8.00

$-1.00$

8.00

0.00

8.00

0.00

9.37

$-0.37$

13.15

1.85

15.00

0.00

SS $=91.677$

Figure 17. Predicted Values and Residuals Using Forward Rank Regression and Backward Rank Regression. 


\section{$\underline{\text { References }}$}

Brownlee, K. A. (1965). Statistical Theory and Methodology in Science and Engineering, 2nd ed. John Wiley and Sons, Inc., New York.

Conover, W. J. (1980). Practical Nonparametric Statistics, 2nd ed. John Wiley and Sons, Inc., New York.

Draper, N. R. and Smith, H. (1966). Applied Regression Analysis. John Wiley and Sons, Inc., New York.

Iman, Ronald L. and Conover, W. J. (1979). The Use of the Rank Transform in Regression. Technometrics, Vol. 21, No. 4, pp. 499-509.

Iman, Ronald L. and Conover, W. J. (1980). Risk Methodology for Geologic Disposal of Radioactive Waste: A Distribution Free Approach to Inducing Rank Correlation Among Input Variables for Simulation Studies, NUREG/CR-1262.

Iman, Ronald L., Davenport, James M., Frost, Elizabeth L. and Shortencarier, Michael J. (1980). Stepwise Regression with PRESS and Rank Regression (Program User's Guide). Technical Report, SAND79-1472, Sandia Laboratories, ATbuquerque.

Daniel, Cuthbert, and Wood, Fred S. (1971). Fitting Equations to Data. John Wiley \& Sons, Inc., New York. 


\section{EXAMPLE OF SETTING UP AND EXECUTING THE LATIN HYPERCUBE SAMPLING PROGRAM ALONG WITH OUTPUT FROM A TRANSPORT MODEL}

This part of the course demonstrates the techniques of the first two sections on the NWFT/DVM model. The NWFT/DVM model uses 17 input variables, 4 of which are correlated.

Page 104 shows the parameter cards used to generate the Latin hypercube sample as described in SAND79-1473.

Page 105 gives the user specified subroutine as required on cards 14 to 21 on page 104 .

Pages 106-108 give the actual LHS for 17 variables and a sample of size 35 .

Pages 109-111 give the ranks from 1 to 35 for each of the 17 input variables on pages 106-108.

Page 112 contains the output from running the 35 input vectors through NWFT/DVM. The output is in the form of total integrated discharge for 7 isotopes over $10^{4}$ years on a per vector bas is. 
Card No.

1 .

2 .

3.

4.

5.

6.

7.

8.

9.

10 .

11 .

12 .

13.

14.

15.

16 .

17.

18 .

19.

20.

21 .

22 .

23.

24 .

25 .

26 .

27.

28.

29 .

30 .

31 .

32 .

33.

34.

35 .

36 .

37.

38 .

39 .

40 .

41 .

42 .

43.

44 .

45 .

46 .

47 .
$624576 \geq 762631657$

LHS SCENARIOS NHFT OVM

TITLE-LHS NHFT CUM FOR NRC SHORT CCLRSE

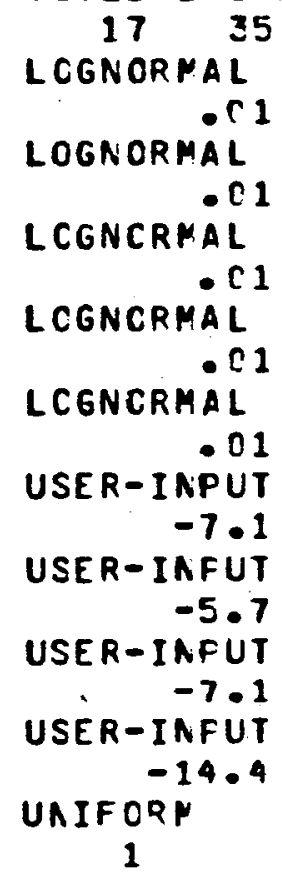

LCGUNIFORN

50.

1

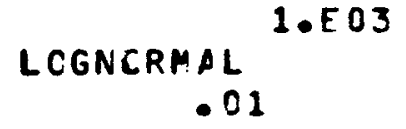

NCRMAL

- CS

LOGUNIFCRP

1

1

NCRMAL

UAIFOR ${ }^{.05}$

1

UNIFORN

1

4

$\begin{array}{llll}12 & 13 & 14 & 15\end{array}$

$\begin{array}{llll}1.0 & 0.7 & 1.0 & 0.0\end{array}$

$0.7 \quad 1.0$

100

.05

$$
\begin{gathered}
\text { 1. E5 KO FOR CM(AM) } \\
\text { KD FOR PU }
\end{gathered}
$$

$1 . E 4$

1.E4 KD FOR U

KD FOR TH

$1 \cdot E 4$ KD FOR NP 50. SOL LIMIT FOR PJ(LOE 1O) 2. SOL LIMIT FOR U!LOG 10) 1 . SOL LIMIT FOR TH(LOE 10) .6 3 . SOL LIMIT FOR NP(LOG 10) DISPERSI VITY

500.

LEACH TIME

$1 \cdot[C]$ 5n.

KIUPPER AQUIFERI .30

POROSITY (UPPER AOUIFER)

$K$ OF THE FEATUR: (S) .30

25.

POROSITY OF THE FEATURE(S)

TIME OF ONSET OF MIGRATION

$1 \cdot E 4$

NUMBER OF ROOMS

1100 .

OUTPUT, OATA,FLOT, CORR 
EXAMPLE OF A USER SUPPLIED SUBROUTINE FOR USE WITH THE LATIN HYPERCUBE SAMPLING PROGRAM TO GENERATE A SAMPLE FROM A DISTRIBUTION NOT INCLUDED IN THE PROGRAM - THE IMPLEMENTATION OF THIS SUBROUTINE IS EXPLAINED IN THE PROGRAM USER'S GUIDE

SUBRCUTINE USKDISTII,N,INSET,IRS DLI,L2,LI)

COMMON L(1000C)

COMPCN/A/X(100000)

CCMNON/B/XX(100000)

LE VEL $2, x, \times X$

$\operatorname{LOC}(I, J)=(7-1) \cdot N+I$

ALPINC $=0.95 / N$

REAC $(8,11 C) A, B$

110 FORMAT (2G1C.4)

C A IS THE MEAN ON A LOGIO SCALE FER NORMAL DISTRIBUTION

C B IS THE ST CEV ON A LOGIO SCALE FOR A NORMAL DISTRIBUTION

C FOR THIS DISTRIBUTION A WILL GE TREATED AS THE O.J25 OUANTILE

C AND B HILL BE TREATED AS THE O.915 OUANTILE

CELTA $=.025$

CO $10 \quad K=1, N$

$R=A L P I N C \otimes U D G E N(0)+.D E L T A$

$X(L O C(K, I))=10 * *(F I N V N O R(R) * B+A)$

CELTA= CELTA+ALP INC

10 CONTINUE

IF (INSET ANE.1)GO TO 20

FR INT $12 C, I, A, B$

FRIAT $130, L 1, L 2, L 3$

20 CONTINUE

120 FORYATC*C*,11X,I3,12X,*USER SUPPLIED MEAA $=*, 1 P G 10.3$,

* $\quad$ ST DEV $=\star, 1 P G 10.3)$

130 FORYAT (1H+.75X,3A1D)

RE TURN

END 
TITLE-LHS MWFT DVM FOR NRT SHORT COURSE

INPUT VECTORS

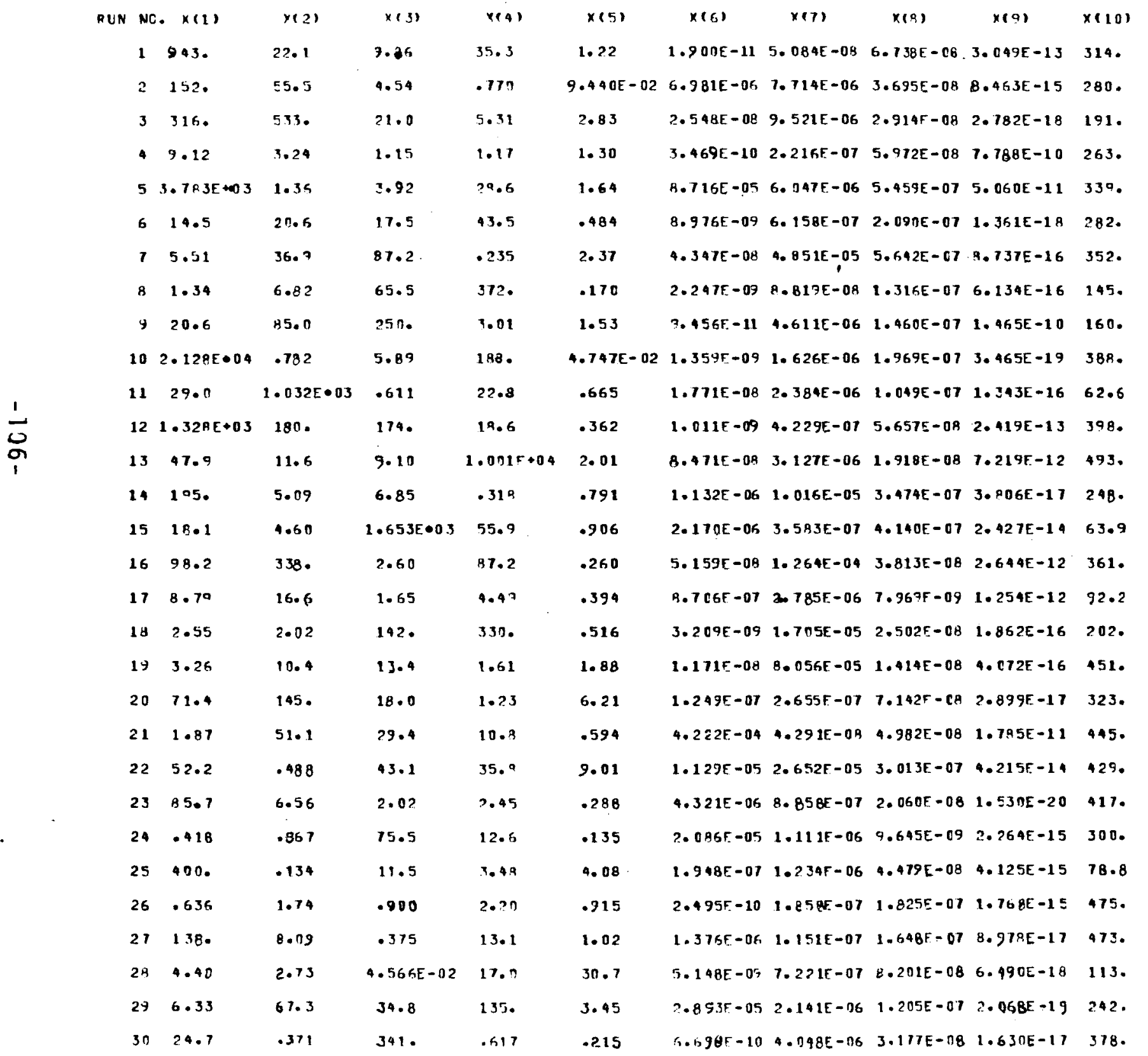

$x(11) \times 112$

$1.152 E .003 \quad 7.913 E-02$

$4.481 E \cdot 03 \quad .209$

$2.076 E+044.51$

$5.538 E \bullet 04 \quad .355$

2.077E+06 8.83

$2.276 E+03 \quad 12.6$

$1.739 \mathrm{E} \bullet 03 \quad 1.05$

$1.4895006 \quad .580$

$4.6395005 \quad .831$

$2.999 E \bullet 04 \quad 1.51$

$6.595 E \cdot 05 \quad .116$

$1.632 E+06 \quad 1.14$

$2.3565 \cdot 05 \quad 2.66$

3.169506 .316

$B .6305 \cdot 03 \quad .145$

$7.106 E \div 03 \quad .187$

$2.824 E+05 \quad 1.82$

$7.184 E \cdot 06 \quad .709$

$3.773 E+05 \quad 1.68$

$1.162 E+06 \quad 1.28$

$3.138 E \bullet 04 \quad .248$

$1.517 E \cdot 053.619 E-02$

$1.191 E \bullet 05 \quad .27$ म

$1 .+85 E \bullet 04 \quad 2.27$

$1.251 E+04 \quad 5.75$

$3.8795+06 \quad .409$

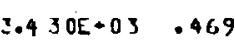

$7.8665+05 \quad .653$

$4.998 E+04 \quad .914$

$1.123 E+05$ B. 11 QE-0? 


\begin{tabular}{lllll}
1 & 31 & 11.1 & 29.5 & 1.26 \\
\hdashline & 32 & 586. & 30.2 & 5.04 \\
1 & 33 & 250. & 4.02 & 37. \\
& 34 & .160 & 13.1 & .206 \\
& 35 & 35.2 & .217 & 2.43
\end{tabular}

$\begin{array}{lllllllll}1.53 & 7.578 E-02 & 4.093 E-07 & 1.483 E-05 & 9.187 E-07 & 5.662 E-1 \leq & 224 . & 8.492 E+06 & -942 \\ 6.43 & .685 & 0.679 E-07 & 1.471 E-06 & 1.509 F-08 & 7.960 F-14 & 132 . & 5.073 F+06 & .528 \\ 1.872 E-02 & .113 & 3.042 E-07 & 4.911 E-078.457 E-08 & 1.030 E-13 & 212 . & 8.508 E+04 & 2.09 \\ 8.43 & .232 & 1.632 E-075.397 E-06 & 2.489 E-07 & 0.395 E-13 & 172 . & 1.387 E+03 & 3.24 \\ 68.8 & .442 & 2.025 E-08 & 2.023 E-05 & 9.739 E-08 & 1.899 E-14 & 116 . & 6.135 E+03 & .392\end{array}$


TITLE-LHS NUFT DVF FOR NRC SHORT COURSE

INPUT VECTORS

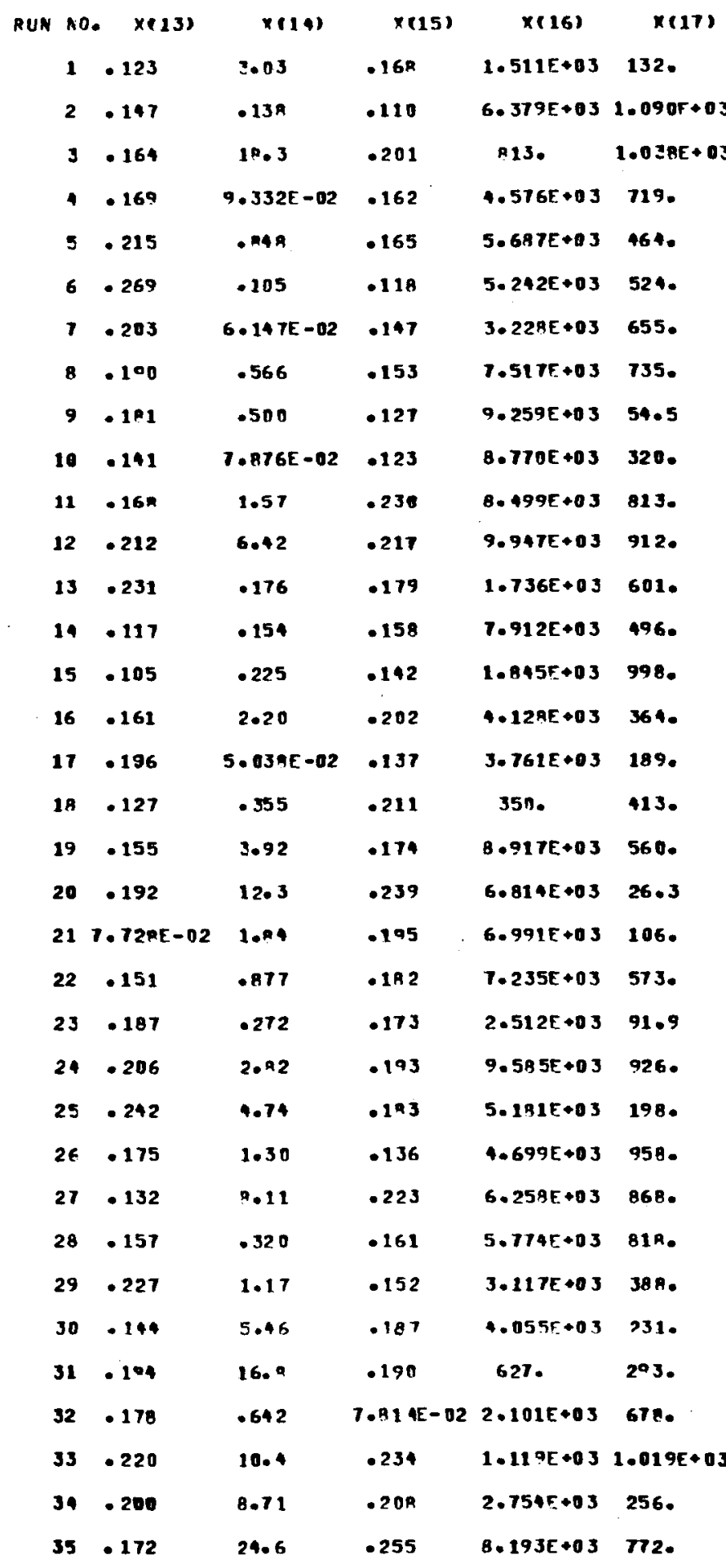


TITLE-LHS NUFT DUP FOR VRC SHOFT CCURSE

\begin{tabular}{|c|c|c|c|c|c|c|c|c|c|c|c|c|c|}
\hline PUN NO. & $x(1)$ & $x(2)$ & $x(3)$ & $x(4)$ & $x(5)$ & $x(6)$ & $x(7)$ & $x(8)$ & $x(9)$ & $x(10)$ & $x(11)$ & $x(12)$ & \\
\hline 1 & 32. & 23. & 18. & 25. & 23. & 10 & 2. & 16. & 27. & 21. & 1. & 2. & \\
\hline 2 & 26. & 29. & 13. & 5. & 3. & 30. & 26. & 10. & 20. & 18 & 6. & 1. & \\
\hline 3 & 29. & 34. & 23. & 14. & 30. & 14. & 27. & R. & 5. & 11. & 12 & 32. & \\
\hline$\bullet$ & 12. & 11. & 6. & 6. & 24. & 9. & 6. & 15. & 35. & 17. & 16. & 11. & \\
\hline 5 & 34. & 1. & 12. & 24. & 26. & 34. & 25. & 33. & 33. & 23. & 30. & 34. & \\
\hline 6. & 1.4. & 22. & 21 & 27. & 14. & 11. & 11. & 28. & 4. & 19. & 9. & 35. & \\
\hline 7 & 9. & 26. & 30. & 2. & 29. & 16. & 3.3 & 34. & 15. & 24. & 3. & 22. & \\
\hline 8 & 4. & 15. & 28 & 34. & 6. & 9. & 3. & 23. & 19. & B. & 29. & 16. & $r$ \\
\hline 9 & 16. & 30. & 33. & 11. & 25. & 2. & 23. & 24. & 39. & 9. & 24. & 20. & 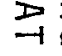 \\
\hline 10 & 35. & s. & 15. & 32. & 1. & 7. & 17. & 27. & 3. & 27 & 13. & 25. & $\ddot{z}$ \\
\hline 11 & 18. & 35. & 4. & 23. & 17. & 13. & 19. & 21. & 11. & 1. & 25. & 4. & $I$ \\
\hline 12 & 33. & 32. & 32. & 22. & 11. & 6. & 9. & 14. & 26. & 20. & 29. & 23. & ס \\
\hline 13 & 20 & 13. & 11. & 35. & 28. & 18. & 21. & 5. & 31. & 35. & 21 & 30. & ग0 \\
\hline 24 & 27. & 14. & 16. & 3. & 19. & 26. & 28. & 31. & 9. & 16. & 31 & 10. & $\underset{\infty}{\subset}$ \\
\hline 15 & 15. & 13. & 35. & 28. & 20. & 28. & B. & 32. & 22. & 2. & 9. & 5. & $m ;$ \\
\hline 16 & 24. & 33. & 10. & 30. & 9. & 17. & 35. & 11. & 30. & 25. & a. & 6. & $\tilde{D}$. \\
\hline 17 & 11 & 21. & 8. & 13. & 12. & 25. & 20. & 1. & 29. & 9. & 22 & 27. & 3 \\
\hline 18 & 6. & 9 & 31 & 3.3. & 15. & 9. & 30. & 7. & 12. & 12. & 34. & 18. & 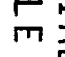 \\
\hline 19 & 7. & 19. & 20. & B. & 27. & 12. & 34. & 3. & 13. & 32. & 23. & 26. & \\
\hline 20 & 22. & 31. & 22. & 7. & 33. & 19. & 7. & 17 & 8. & 22. & 27 & 24. & \\
\hline 21 & 5. & 27. & 24. & 19. & 16 & 35. & 1. & 13. & 32. & 31 & 19. & 9. & \\
\hline 22 & 21 & 4. & 27. & 26. & 34. & 31. & 32. & 30. & 23. & 30. & 20 & 1. & \\
\hline 23 & 23. & 15. & 9. & 10. & 10 & 29. & 13. & 6. & 1. & 29. & 19. & 9. & \\
\hline 24 & 2. & 6. & 29. & 19. & 5. & 32. & 14. & 2. & 17. & 20. & 11 & 29. & \\
\hline 25 & 30. & 1. & 19 & 12 & 32. & 21. & 15. & 12. & 18. & 3. & 10 & 33. & \\
\hline 26 & 3. & 9. & 5. & 9. & 21 & 3. & 5. & 26. & 16. & 34. & 32. & 13. & \\
\hline 21 & 25. & 17. & 3. & 20 & 22 & 21. & •. & 25 & 10 & 33. & 5. & 14. & \\
\hline 28 & A. & 10. & 1. & 21 & 35. & 10 & 12. & 19. & 6. & 5. & 26. & 17. & \\
\hline $2 y$ & 10. & 29 & 25. & 31 & 31. & 33. & 15. & 22. & 2. & 15. & 15. & 10 & \\
\hline $3 n$ & 11. & 3. & 34. & 4. & 7. & 5. & 22 & 9. & 7. & 35. & $1 \therefore$ & 3. & \\
\hline
\end{tabular}




\begin{tabular}{|c|c|c|c|c|c|c|c|c|c|c|c|c|}
\hline & 31 & 13. & 24. & 7. & 15. & 2. & 23. & 29. & 35. & 19. & 14. & 35. \\
\hline & 32 & 31. & 25. & 14. & 15. & 19. & 24. & 16. & 4. & 24. & 7. & 33. \\
\hline I & 33 & 2R. & 12. & 26. & 1. & 4 & 22. & 10. & 19. & 25. & 13. & 17. \\
\hline 5 & 34 & 1. & 20. & 2. & 17. & R. & 20. & 24. & 25. & 2R. & 10. & 2. \\
\hline & 35 & 19. & 2. & 11. & 29. & 13. & 15. & 31. & 20. & 21. & 6. & 7. \\
\hline
\end{tabular}


TITLE-LHS MHFT DVÁ FDR NRC SHORT COLRSE

nakiks of

INPUT VECTORS

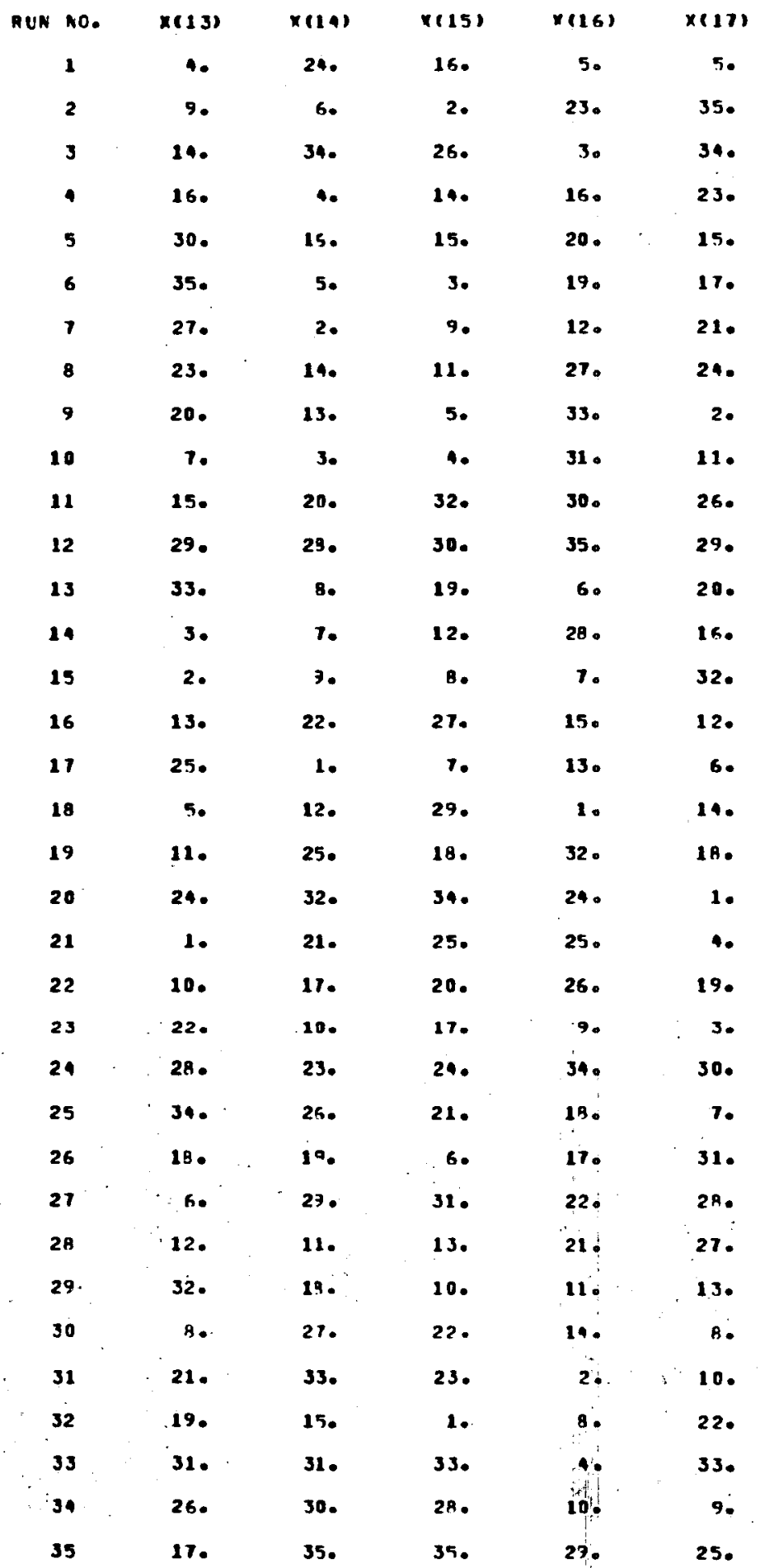




\section{SAMPLE OUTP.UT GENERATED FROM A TRANSPQRT}

MODEL USING THE PREVIOUS LATIN HYPERCUBE SAMPLE AS INPUT

Total Integrated Discharge to $10^{4}$ Years

Run

No.

${ }^{237} \mathrm{~Np}$

$233 u$

${ }^{229} \mathrm{Th}$

${ }^{246} \mathrm{Cm}$

$242 p u$

$238 U$

$238 \mathrm{pu}$

10.

0.0 .

4.809E-05 3.997E-09 0.

5.807E-02

2.110E-04

8.775E-03

0.

0.

4.066E -07

$3.740 \mathrm{E}-08$

1.311E-06

0.

0.

$1.329 \mathrm{E}-04$

2.863E-06

0 .

0.

0.

0 .

0.

2.385E-04

0.

0.

3.097E-05

0 .

0 .

5 8.083E-06

$1.214 \mathrm{E}-06$

1.101E-06

2.403E- 02

9.049E-05

$2.754 \mathrm{E}-10$

0.

1.268E-05 0 .

$6 \quad 5.576 \mathrm{E}-04$

2.207E-08

8.621E-04

6.525E-05

$1.710 E-08$

1.187E-07 0 .

$9.516 \mathrm{E}-07$

$1.721 E-11$

0 .

0 .

0.

0 .

0 .

0 .

0 .

0 .

0 .

5.496E-08

0.

0 .

0 .

$10 \quad 3.740 E-06$

$6.712 E-130$.

110.

$0 . \quad 0$

0.

0.

2.807E-06

3.090E-09

0.

1.054E-07

0 .

0 .

0.

0.

0 .

0.

0 .

0.

4.503E-06

0 .

0 .

0.

0.

150.

0.

0.

$16 \quad 8.823 E-04$

5.075E-07

0.

$5.218 \mathrm{E}-06$

5.379E-07

0.

5.223E-11

4. $088 \mathrm{E}-10$

0.

$7.345 E-06$

1.052

0 .

0.

0 .

20 1.139E-06

$2.458 \mathrm{E}-06$

6.329E-05

$6.568 E-05$

6.829E-06

0.

0.

7.751E-07

0 .

0 .

0 .

210.

$0 . \quad 0$

$0 . \quad 0$.

0.

0.

0.

0.

3.175E-05

$5.000 \mathrm{E}-08$

2.164E-09

0 .

0.0

2.354E-03

0.

24 1.133E-05

$25 \quad 4.759 E-03$

3.276E-05

4.575E-03

0 .

3.387E-07

$1.372 E-02$

0.

6.111E-07

0.

0.

$26 \quad 1.227 \mathrm{E}-06 \quad 7.218 \mathrm{E}-07$

27 4.045E-05

$1.882 E-05$

4.092E -08

1.737E-04

3.003E-07

0 .

0 .

0.

1.936E-07

0 .

0.

$1.138 \mathrm{E}-05$
$1.888 \mathrm{E}-08$

$5.402 E-08$

0 .

0 .

0 .

0 .

0.

8.373E-06 0 .

2.401E-04

0 .

0.

1.349E-06

2.725E-02

0 .

0.

8.097E-03

0

0.

2.289E-10 0 .

3.261E-03

0 .

5.781E-03

0 .

12.2

$1.471 \mathrm{E}-03$

6.242E-06

0 .

2.614E-06

0 .

0 .

8.495E-08 0 .

9.383E-07 0 .

1.077E-06 0 .

$0 . \quad 0$.

0 .

0.

0 .

3.816E-05

0 .

0 .

0 .

0 .

350 .

0 .

0.

6.204E-04

0.

0 . 


\section{AN EXAMPLE OF SENSITIVITY ANALYSIS \\ RESULTS BASED ON THE PARTIAL RANK CORRELATION COEFFICIENT}

Page 114 contains a summary of the partial rank correlation coefficients as given in Equation (19) on page 98 on Part Two of this course. Listed down the left hand side of the table are the 17 input variables from page 104. The heading across the top of the columns identifies 6 output variables, 3 of which are 1 isted on page 112. The numerical entries in in the body of the table can be used to identify the input variables which are dominate in influencing the output. For example the entry of 70 for KD $U$ and $U 233$ means that the $a b-$ solute value of the partial rank correlation coefficient was at least .70 at sometime during the $10^{4}$ year period.

While page 114 shows the PRCCs based on the 35 input vectors on pages 106-108, pages 115 and 116 show results for two additional sets of 35 input vectors. The results are similar on pages 114,115 , and 116.

Page 117 shows the summary for all 105 input vectors pooled together but the individual numerical entries show a PRCC of at least .50 rather than .70 as on pages 114-116. On page 118 the 105 vector results are repeated for PRCCs of at least .70 .

Pages 119-132 provide PRCC plots for each combination of input variable and output variable that have numerical cable entries on page 117 . 
WHERE TABLE ENTRIES OCCUR THE PRCC BETWEEN

THE INPUT VARIABLE (ROW) AND THE OUTPUT

VARIABLE (COLUMN) ACHIEVED AT LEAST THE

LEVEL . 7 OR .8 IN ABSOLUTE VALUE AS INDI-

CATED AT SOME POINT IN TIME OVER THE 10,000

YEAR PERIOD

PRCC LHS-VUFT-DVY US TID-S3-EH2 HLHC1.E4 YRSI NRC SHORT COURSE Output Variables

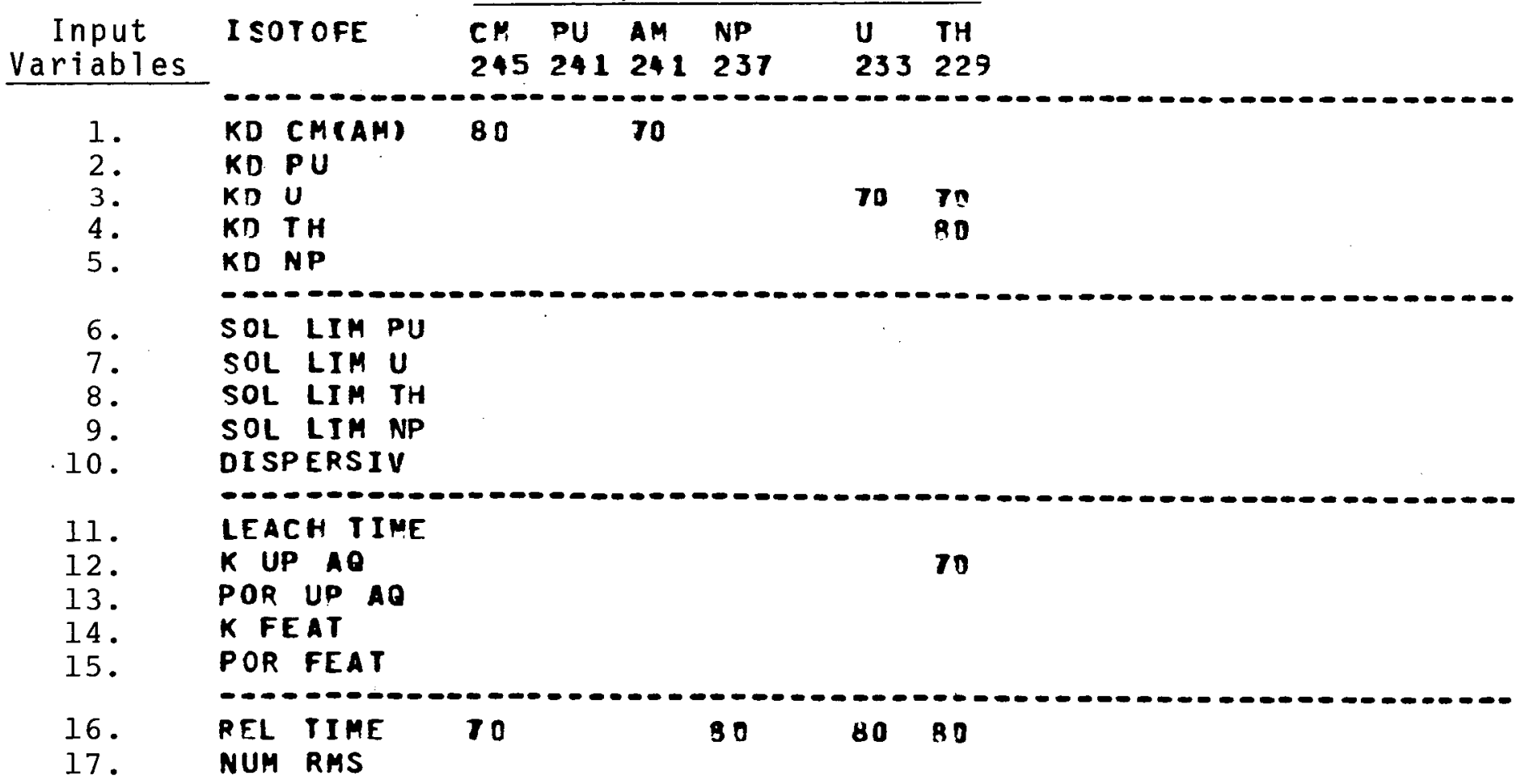


A TABLE SIMILAR TO THE ONE ON PAGE 114 BUT WITH A NEW LATIN HYPERCUBE SAMPLE FOR DEMONSTRATING CONSISTENCY IN IDENTIFICATION OF IMPORTANT VARIABLES

PRCC LHS-NHFT-DVY US TID-S3-CH2 HLH(1.E4 PRS) NRE SHORT COURSE Output Variables

Input ISOTOPE CM PU AM ND U TH Variables

$245241241237 \quad 233229$

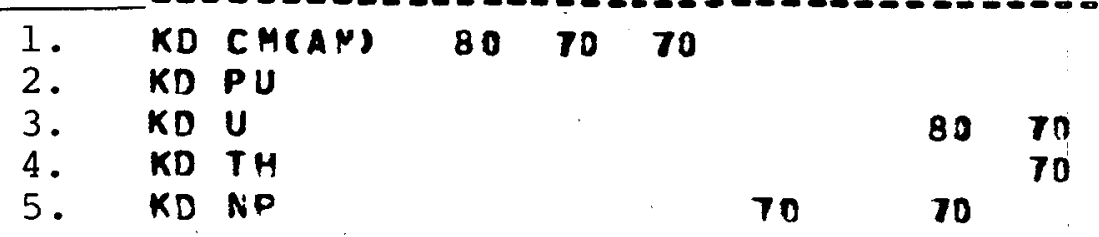

6. SOL LIM PU

7. SOL LIM U

8. SOL LIM TH

9. SOL LIM NP

10. DISPERSIV

11. LEACH TIME

12. K UP AO

13. POR UP AQ

14. K FEAT

15. POR FEAT

16. REL TIME

17. NUM RMS

60

7070

80

8080 
RESULTS FROM A THIRD LATIN HYPERCUBE

SAMPLE TO COMPARE WITH THE TABLES ON

THE PREVIOUS TWO PAGES

PRCC LHS-NHFT-DVM YS TID-S3-CH2 HLH(1.E4 YRSI, NRC SHORT CCURSE

Input

Variables

1 .

2 .

3.

4.

5 .

6.

7.

8.

9.

10 .

11.

12 .

13 .

14 .

15 .

16.

17.

\section{output Variables}

ISOTOPE CM PU AM NP U TH

$\begin{array}{lllll}245241 & 241 & 237 & 233229\end{array}$

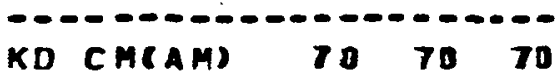

KD PU

$K D U$

Kn TH

KD NP

8D

70

70

SOL LIM PU

SOL LIM U

SOL LIM TH

SOL LIM NP

DI SPEPSIV

LEACH TIME

$K$ UP AO

70

POR UP AO

K FEAT

POR FEAT

REL TIME

NUA RMS $\begin{array}{llll}70 & 70 \quad 89 & 80 \quad 80\end{array}$ 
COMPOSITE RESULTS FROM POOLING ALL COMPUTER RUNS FROM THE THREE PRECEDING LATIN HYPERCUBE SAMPLES WHERE

FILTERS WERE LOWERED TO 0.5 AND .6

DUE TO THE INCREASED SAMPLE SIZE

PRCC LHS-NUFT-DVY VS TID-S3-CH2 HLU(I. EA YRSI NRE SHORT COURSE

Input

Variables

1 .

2 .

3.

4 .

5 .

6 .

7.

8 .

9.

10 .

11 .

12 .

13 .

14 .

15 .

16.

17 .

\section{Output Variables}

\section{SOTOPE}

$$
\text { CH }
$$$$
245241241237
$$

U TH

233227

\section{KD CM(AY) $60 \quad 5060$}

KD PU

KD U

KD TH

KD NP

$60 \quad 50$

60

60

SOL LIM PU

SOL LIM U

SOL LIM TH

SOL LIM NP

DI SPERSIV

LEACH TIME

$K$ UP 10

POR UP AO

K FEAT

PO? FEAT

REL TIME

NUM RMS
60

50

5060

60

60

60 
SAME COMPOSITE RESULTS AS ON PREVIOUS

PAGE ONLY WITH THE FILTERS INCREASED

TO .7 AND .8 FOR PURPOSES ON PINPOINT-

ING DOMINANT VARIABLES

PRCC LHS-NHFT-DVM VS TID-S3-CH2 HLH(I EA YRS) NKC SHORT COURSE

riables

Output Variables

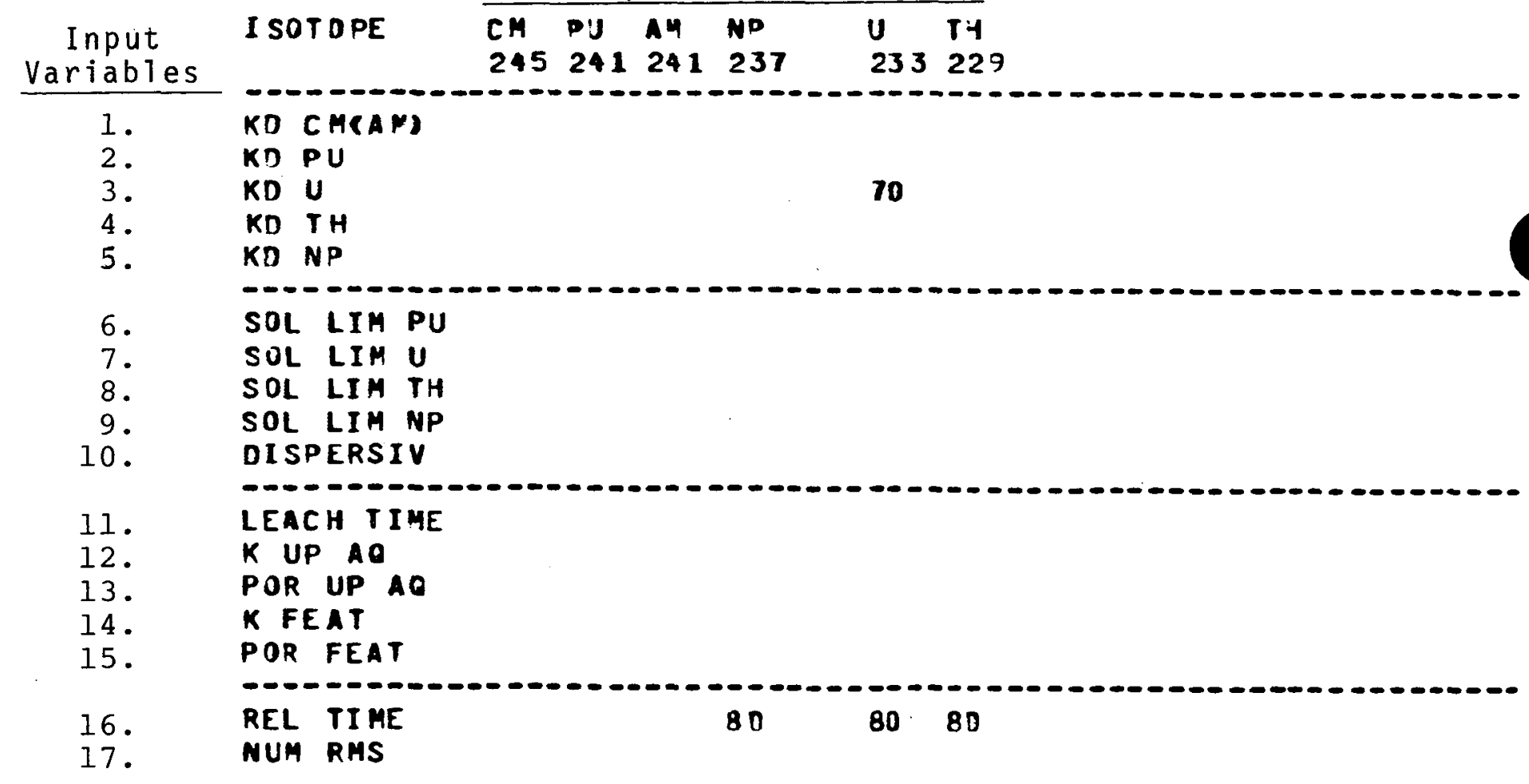




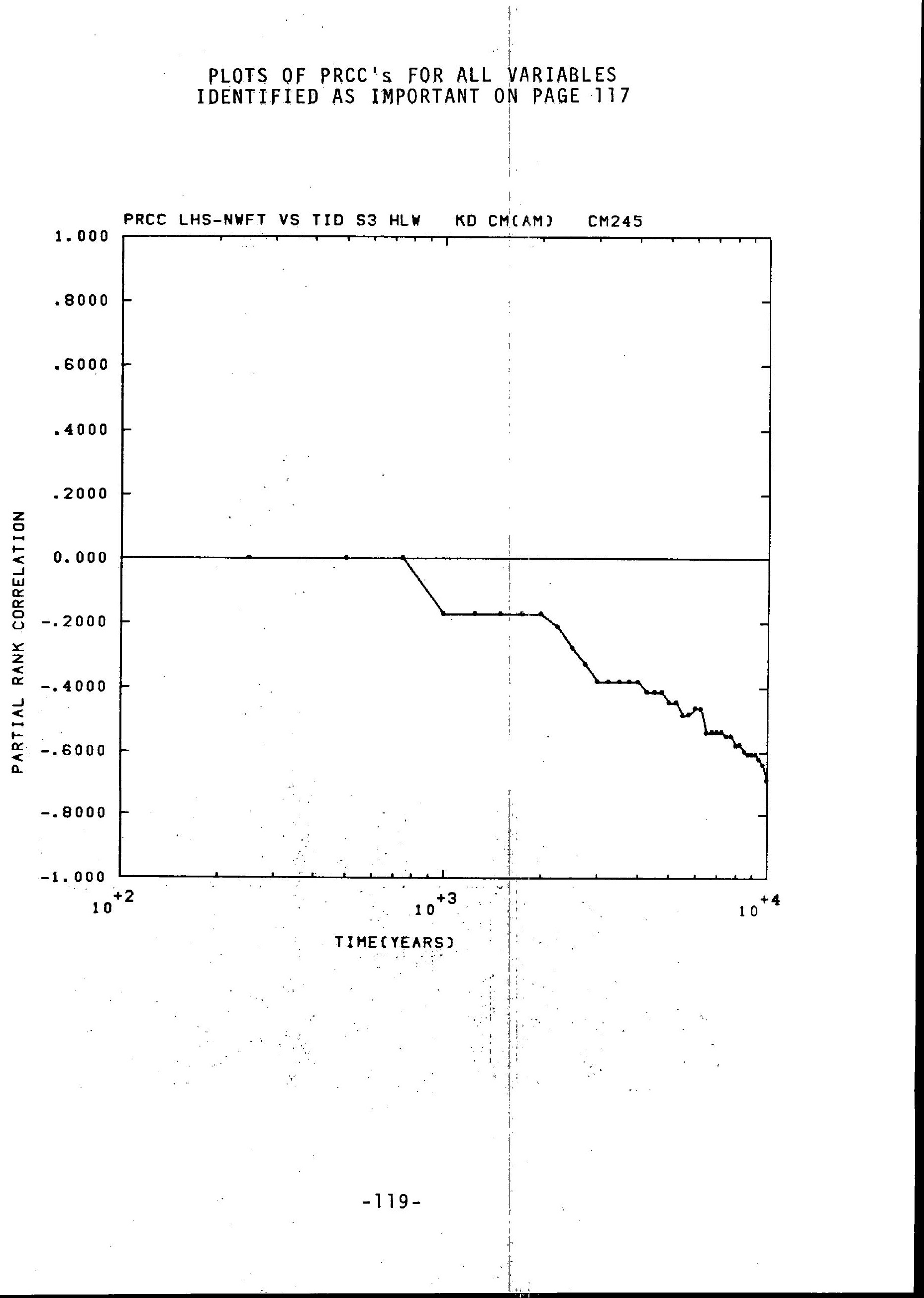




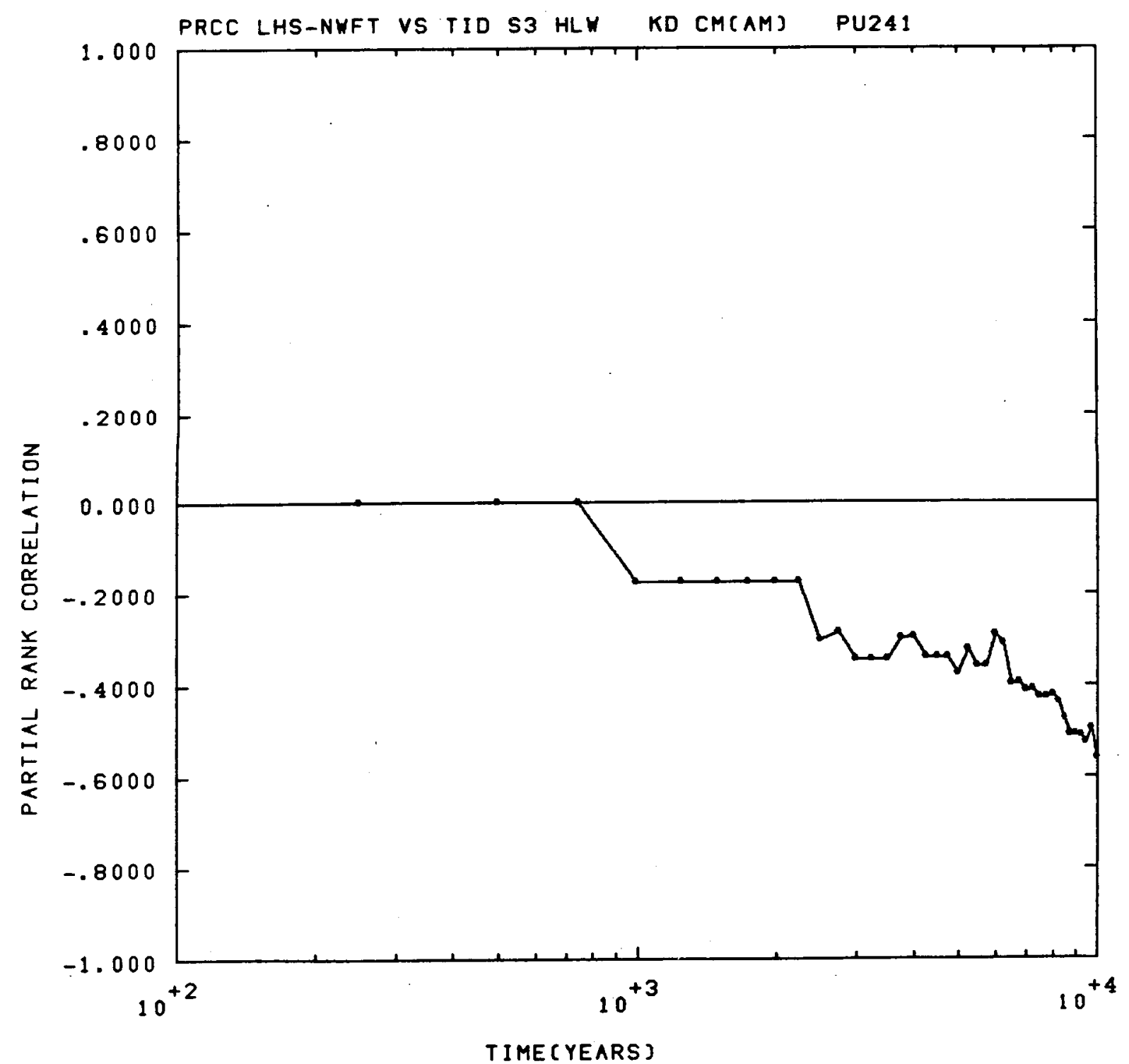




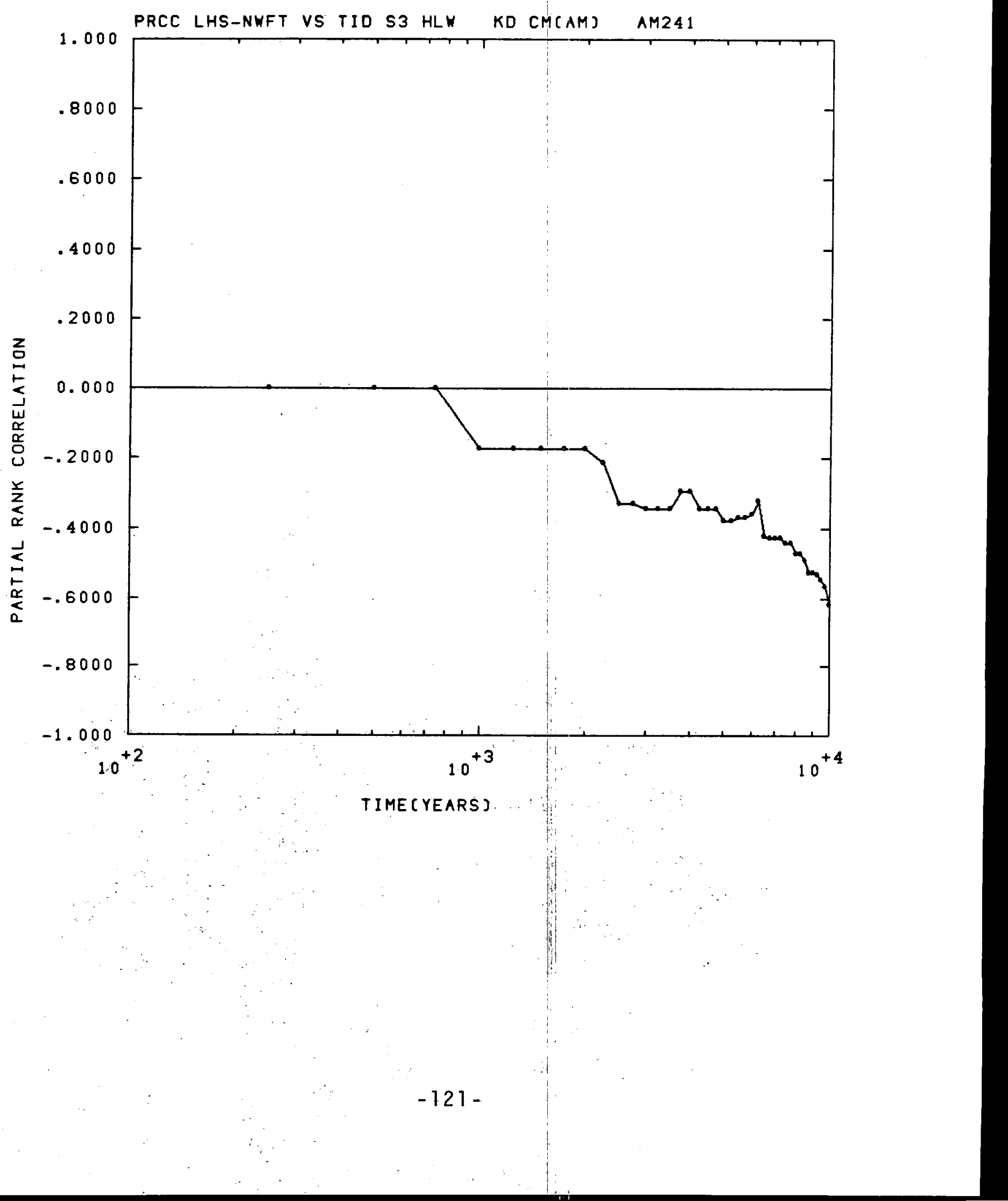




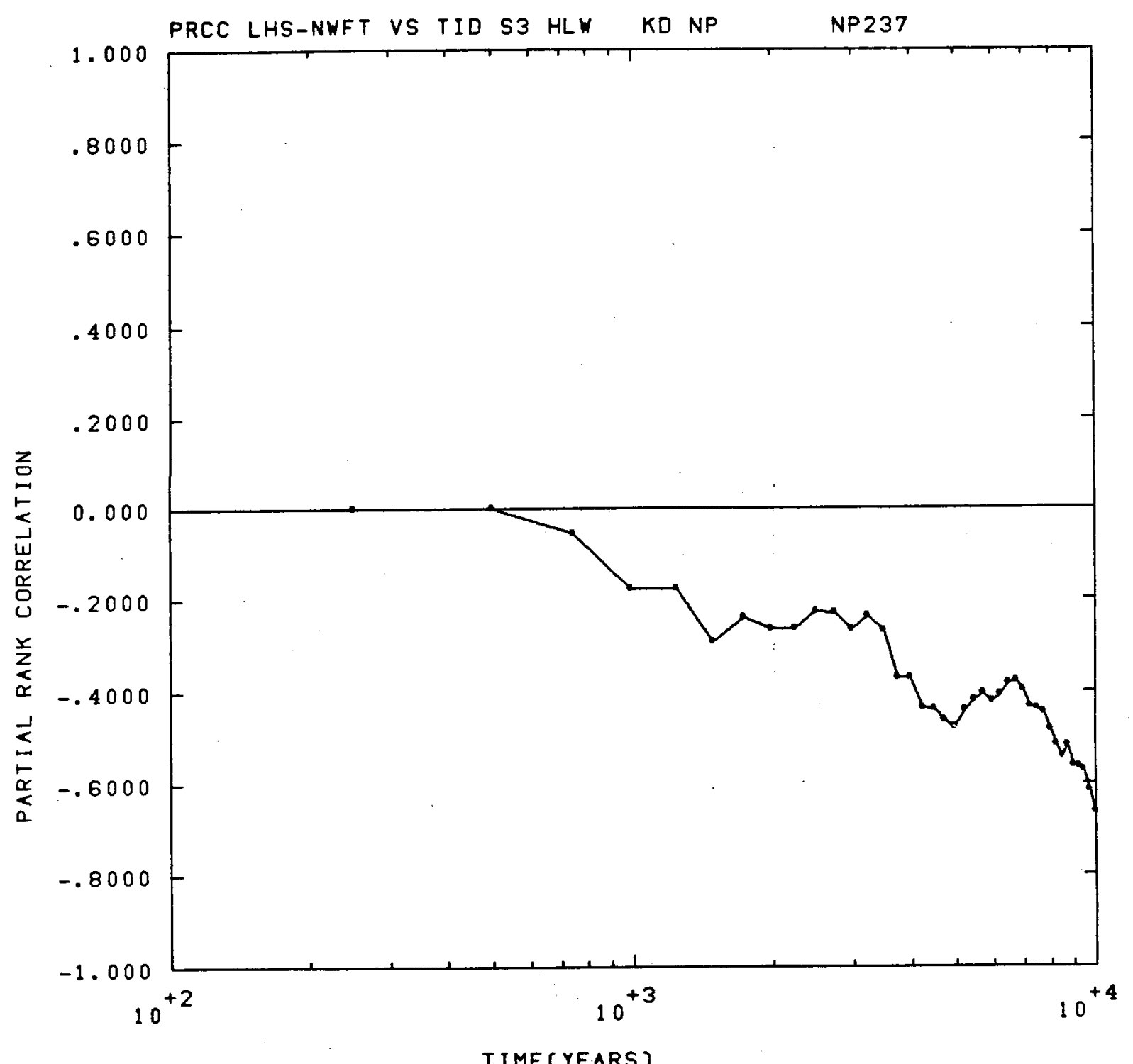

TIME (YEARS) 


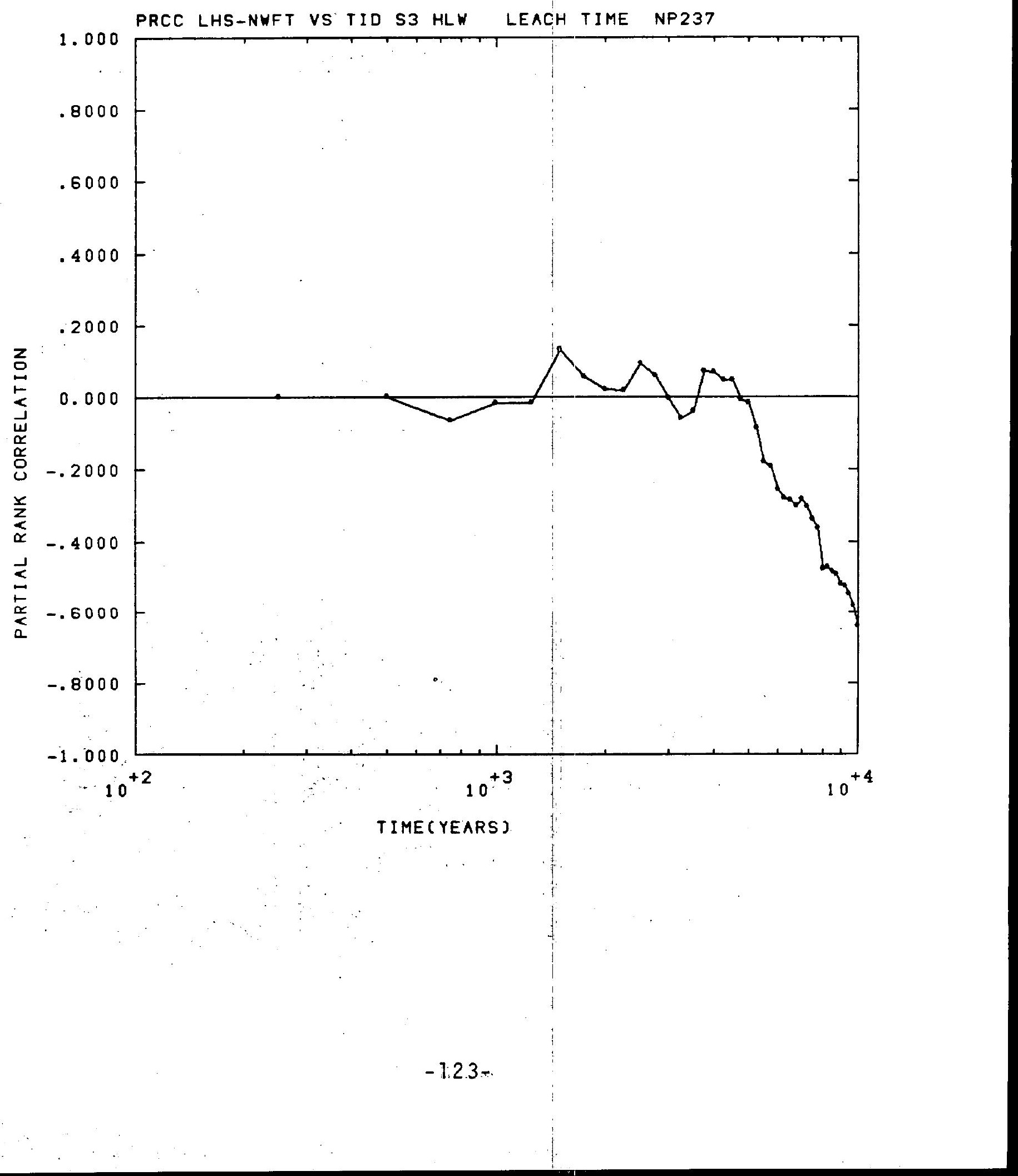




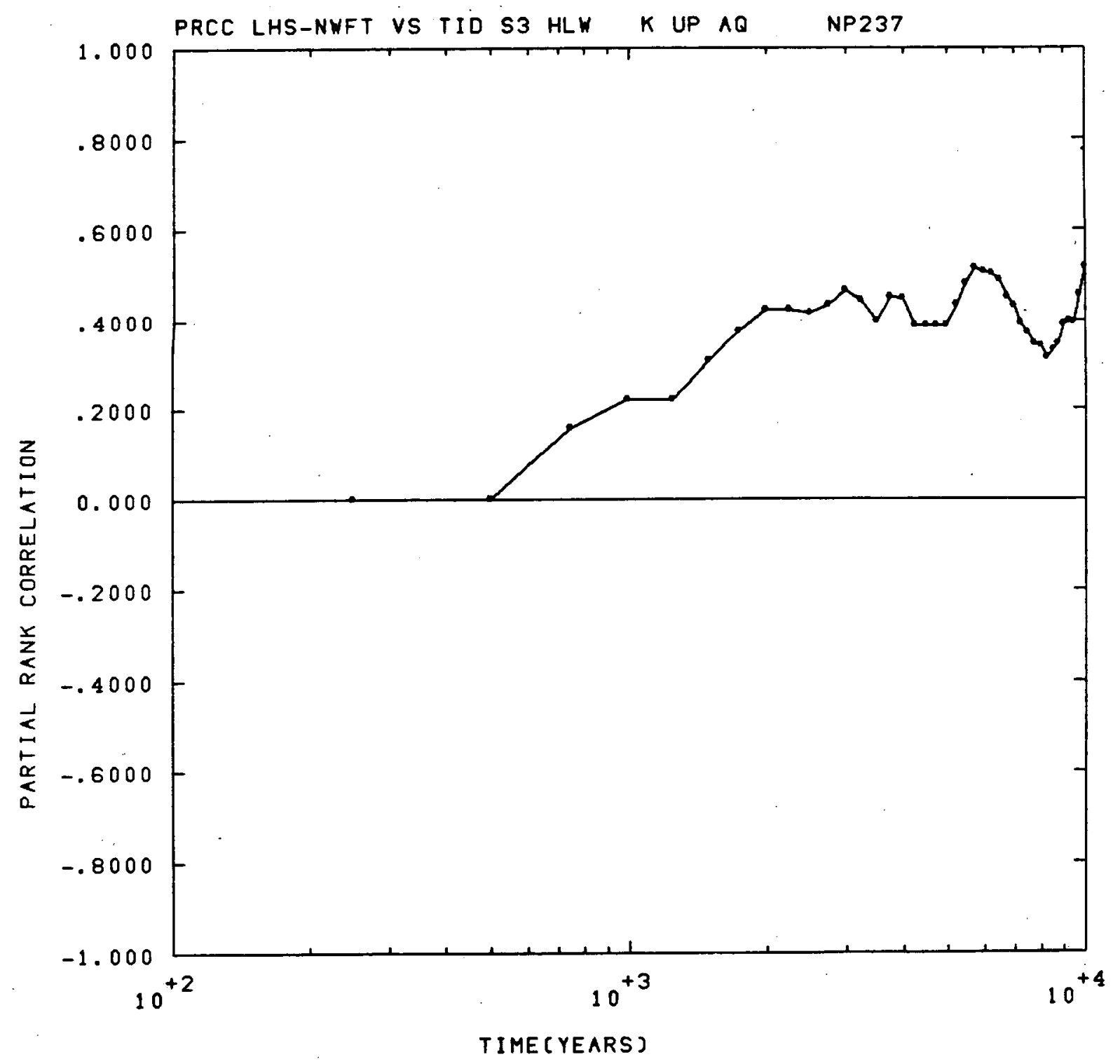




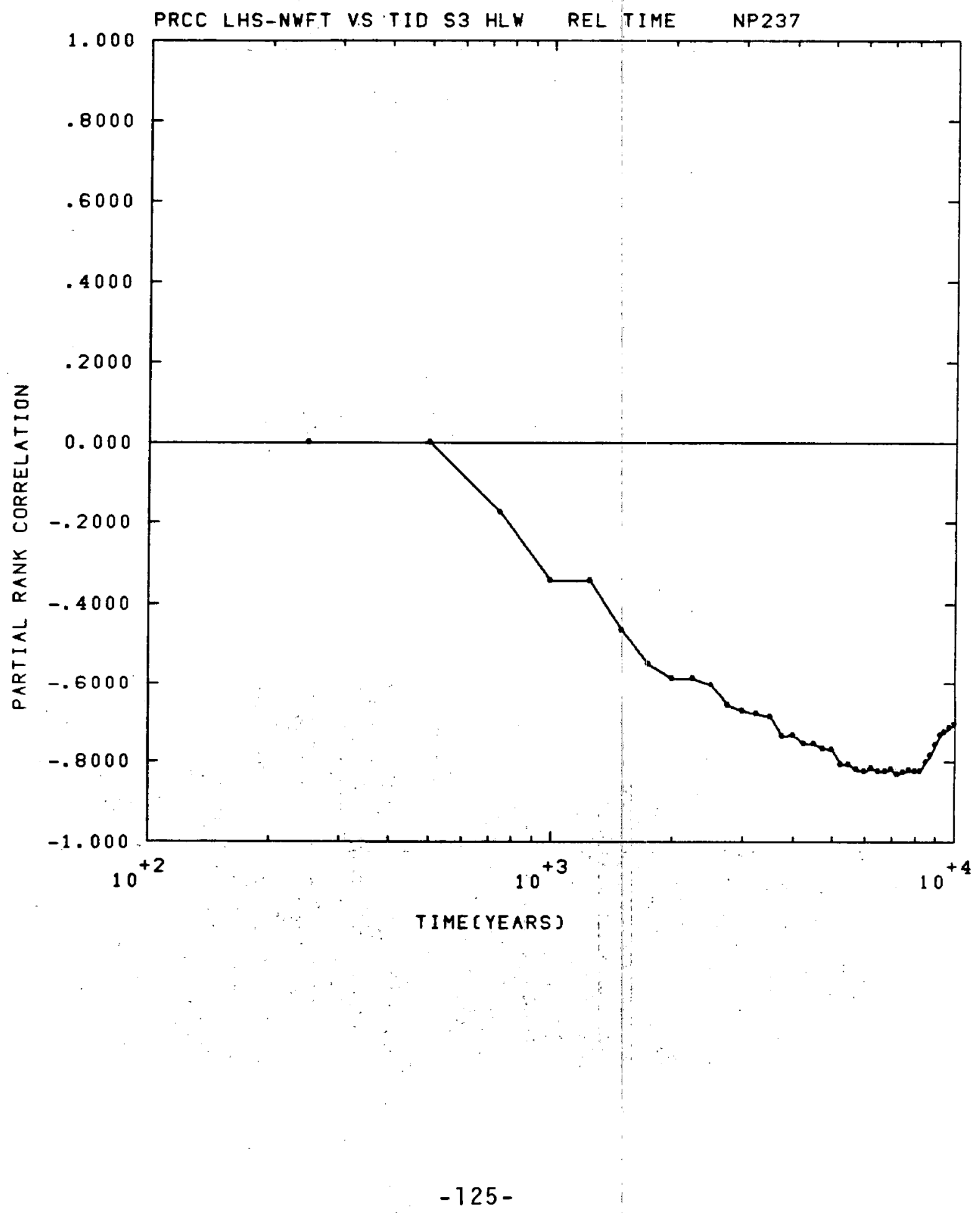




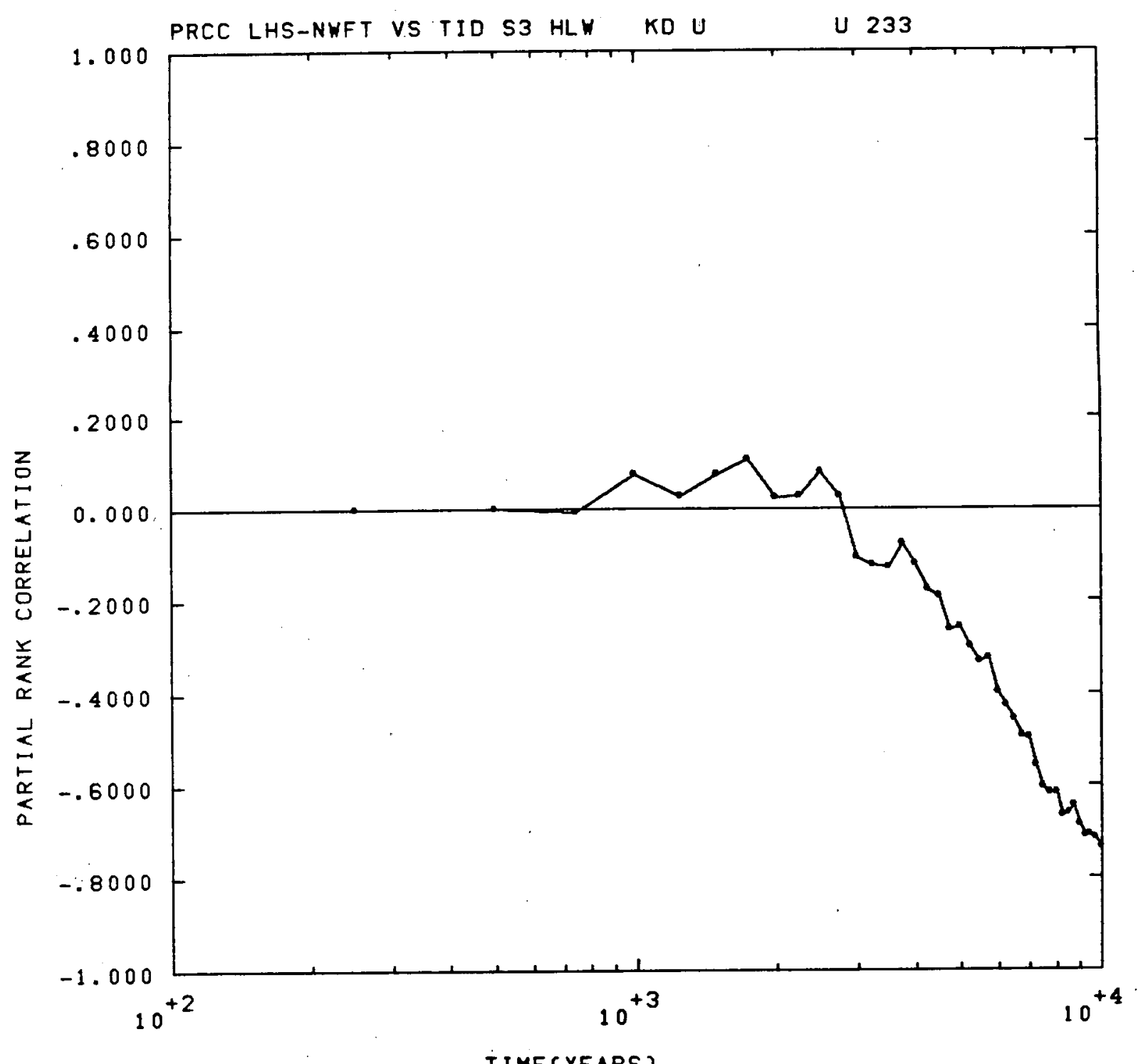

TIME (YEARS ) 


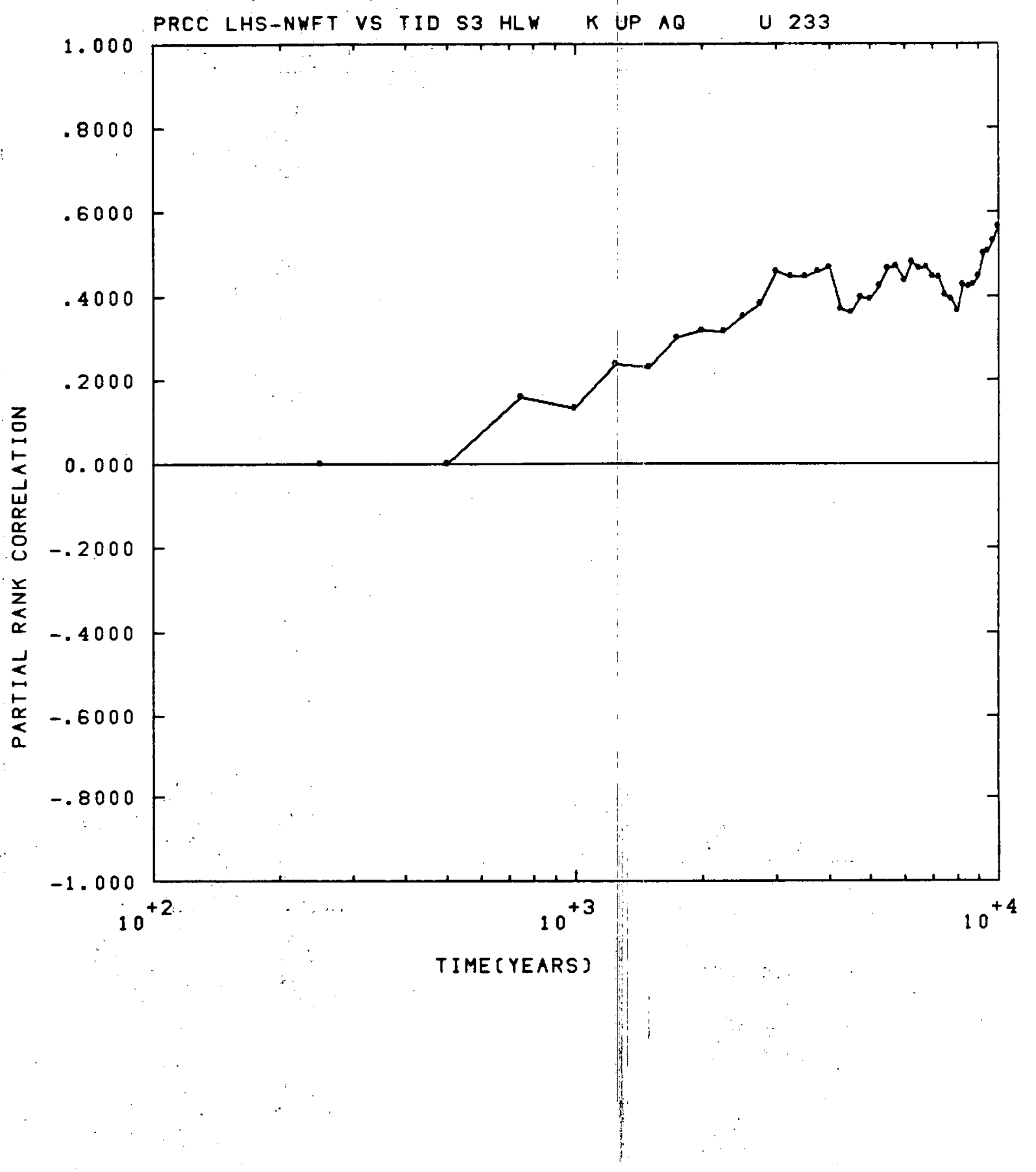




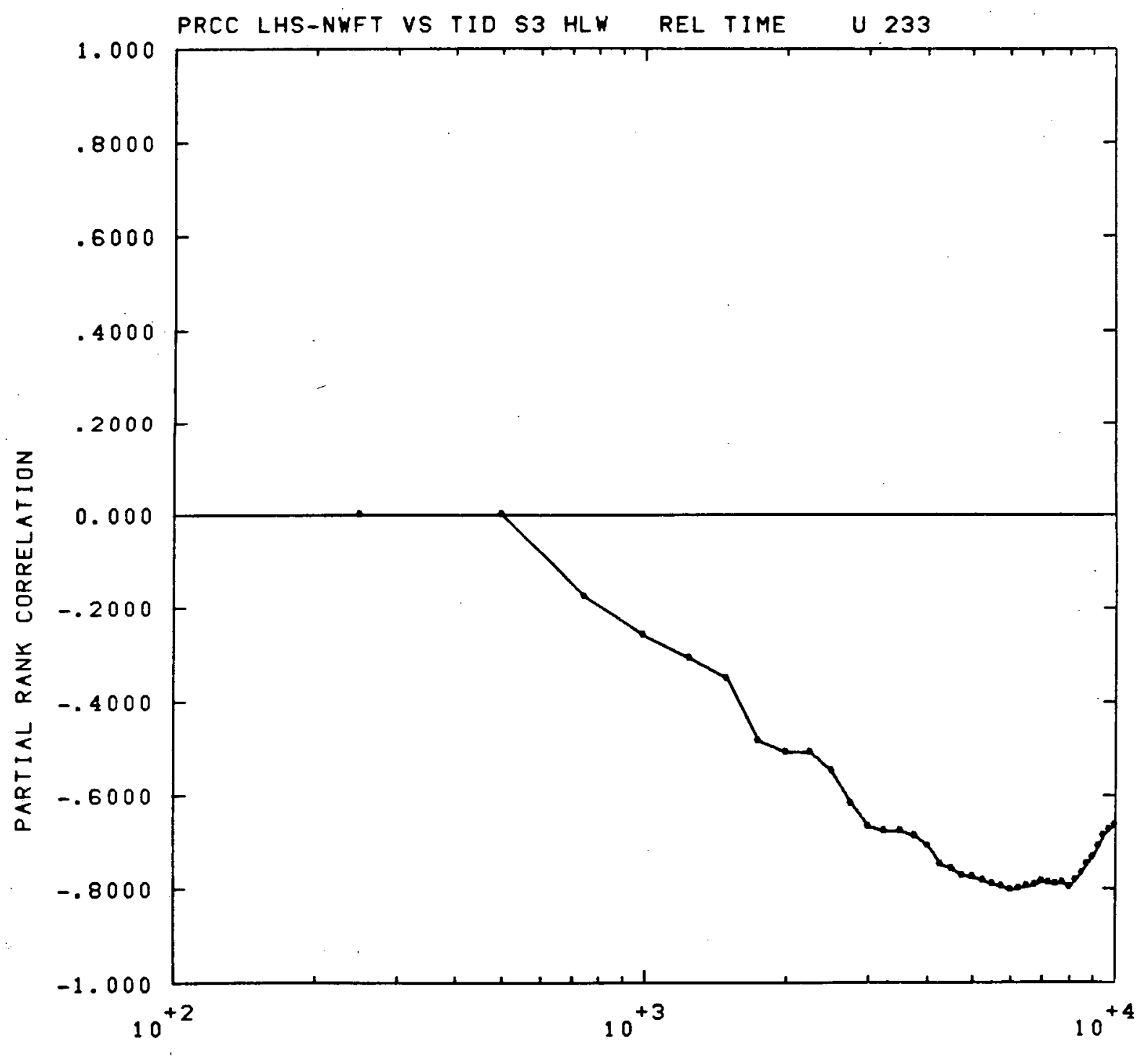

TIME (YEARS) 


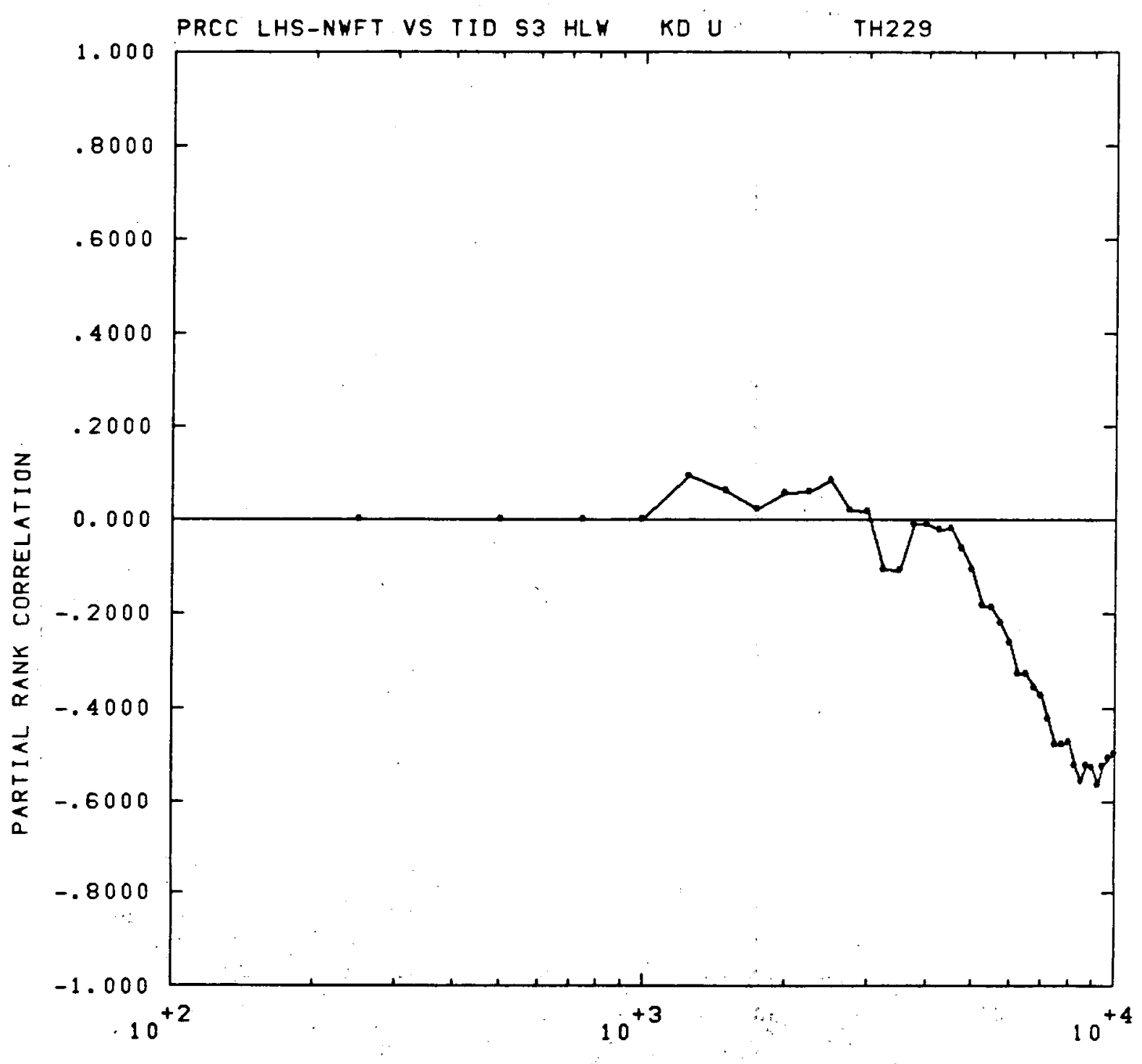

TIME (YEARS) 


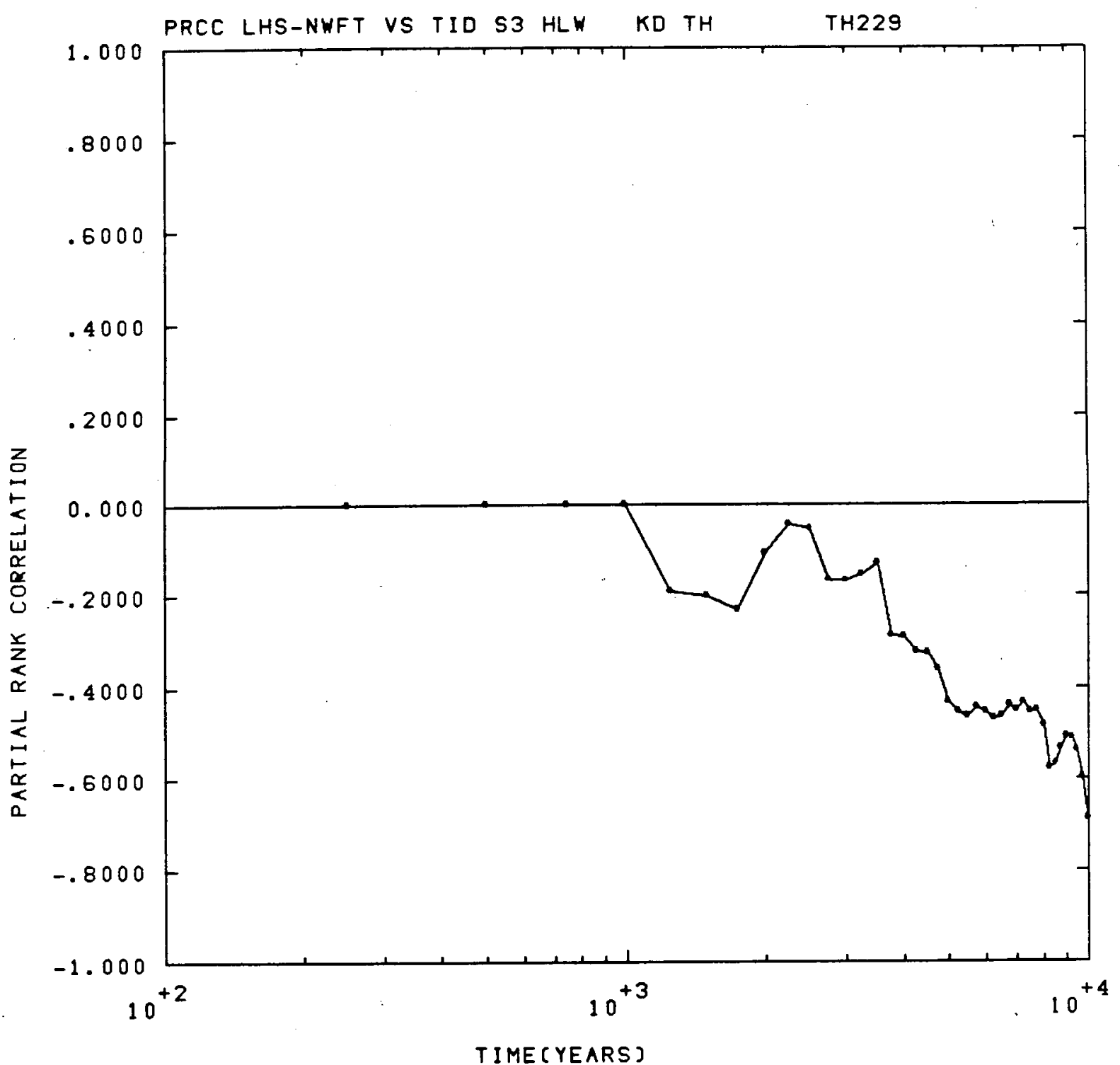




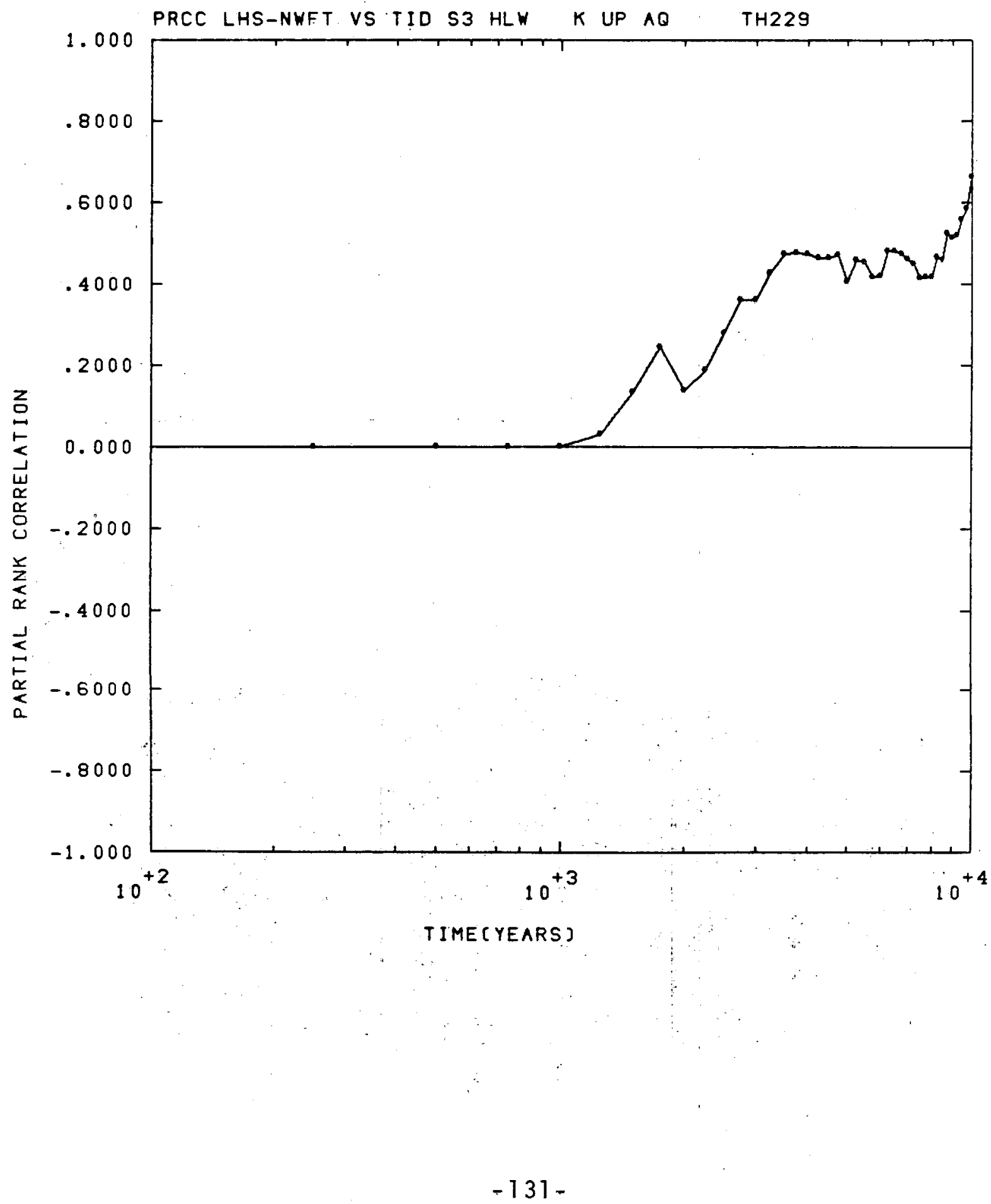




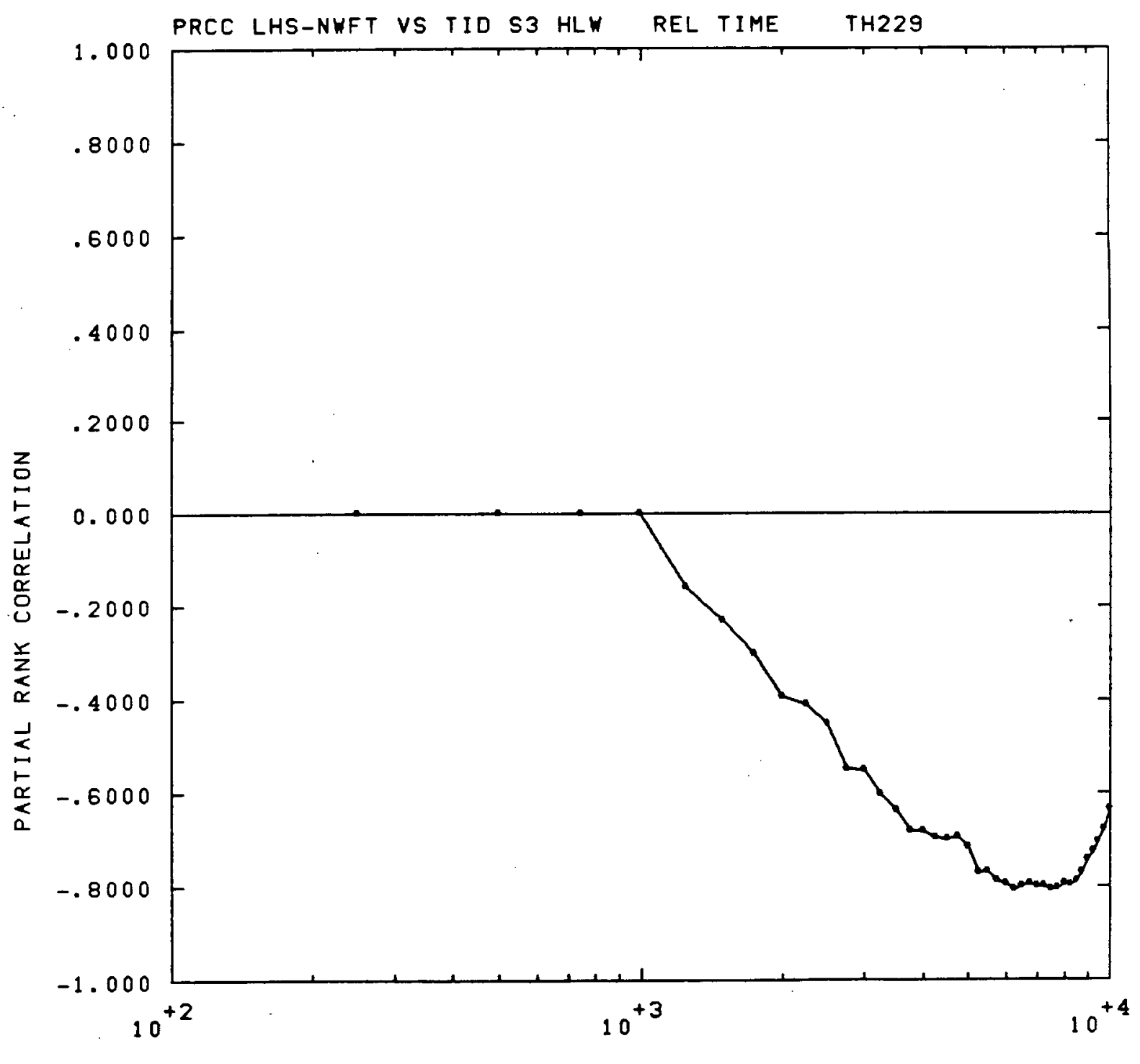

TIME (YEARS) 


\section{STEPWISE REGRESSION ANALYSIS FOR THE PREVIOUS EXAMPLE OF THIS SECTION USING ALL 105 OBSERVATIONS}

The remainder of the pages in this section contain the regression analysis results. Page 134 shows some transformations made outside of the regression program to create new variables such as retardation factors. Page 135 shows transformations for variables made within the regression program. The regression parameter cards as described in SAND79-1472 are listed on page 136 . The results of the regression analysis on raw data for U233 is given on page 137. Pages 138 to 144 contain the results of regression on ranks for U233.

Summaries of the regression results on raw and rank transformed data are given on pages 145 and 146 respectively. An examination of page 145 shows the analysis on raw data to lack consistency of variable selection from set to set and to give poor fits (low $R^{2}$ values). On the other hand the analys is on ranks shown on page 146 does show consistency of variable selection and provides improved fits to the data. 
AN EXAMPLE OF A PROGRAM FOR TRANSFORMING SOME VARIABLES TO CREATE NEW VARIABLES OUTSIDE THE REGRESSION PROGRAM - THESE VARIABLES ARE STORED ON DISK 10 PRIOR TO THE EXECUTION OF THE STEPWISE PROGRAM

PROGRAM STANC INPUT, OUTPUT, TAPE1, TAPE1O) DIMENSION XIN (20), XOUT(25)

$N=1 \cap 5$

DO $100 \quad I=1, N$

READ(1)XIN

$C 0=2.73 *(1.0-X I N(13)) / X I N(13)$

$C E=2.73 *(1.0-X I N(15)) / X I N(15)$

DO $10 \mathrm{~J}=1.5$

$1 \mathrm{C}$.

$L O=2 * J-1$

$L E=2 \star J$

$X O U T(L O)=2 \cdot(i+X I N(J) \cdot C O$

$X O U T(L E)=1 \cdot(+\operatorname{XIN}(J) * C E$

10 CONTINUE

$X O U T(11)=X I N(5)$

15

XOUT $(12)=\operatorname{XIN}(7)$

$X O U T(13)=X I N(A)$

XOUT $(14)=x I N(9)$

$X$ XUT $(15)=X I N(13)$

XOUT $(16)=1 .(1 X I N(11)$

$2 r$

XOUT $(17)=X I N(12)$

XOUT $(18)=1.0 / X I N(13)$

XOUT $(19)=X I N(14)$

XOUT $(20)=x \operatorname{IN}(15)$

XOUT(21) $=$ XIN(15)

25

$x O U T(22)=x I N(17)$

XOUT( 23$)=X I N(18)$

XOUT( 24$)=X I N(19)$

$X O U T(25)=X I N(23)$

HRI TE ( 10$) \times U U T$

30

1OC CONTINUE

REHIND 10

END 
SUBROUTTINE FOR MAKING TRANSFORMATIONS

WITHIN. THE REGRESSION PROGRAM

SUBROUTINE TRAVS( $X)$

COMMON/I MAN/NRAH, NTRAAS, I DROP, IDUM, IRANK DIMENSION $X(49)$

5

DO $1 I=1,22$

$1 \times(I+25)=x(I) \times x(1)$

$x(48)=x(17) * x(18)$

$X(49)=X(19) \div x(2 C)$

RETURN

END 
SANOTA LABORATORIES S STEPUISE REGRESSION FROGRAM C COURTEST OF OEPT. OF STATISTICS - KANSAS. STATE UNIVERSITY TITLE,STEPMISE FOR MRC SHORT COURSE RAN CATA VECT 1-1:S

DATA.25,24.2.

INPUT CHECK OF PARAMETERS

NUHBER OF VARIAHLES REAO IN = 25

NO. OF TRANSF ORMEO VARLABLES $=24$

DATA DISPOSITION IS 2

LABELCIIISF A CW,AF S CM,RF A PU,RF S PU,RF A U,RF S IIRF A THORF S TH, LABEL(9)=RF A NP, RF S MP,S LIM PU,S LIM U,S LIM TH,S LIM MP,DISP,LEACHT, LABEL(1T)=CONO MO,POR MO, CONO S,POR SOREL TIME, NUM RMS,TIO MP,TIO U,TID TH, LARELC26) $=\times 150, \times 250, \times 350, \times 450, \times 550, \times 559, \times 750, \times 850, \times 95 n, \times 1050, \times 1150, \times 1250$, LABELC 38) $=\times 1350, \times 1450, \times 1550, \times 1650, \times 1750, \times 1850, \times 1950, \times 2050, \times 2150, \times 2250$, LABEL(AB) $=\times 17 \cdot \times 18, \times 19 \cdot \times 20$

OUTPUT, CORA, STEPS

STEPUTSE, SIGIN $=0.05$, SI GOUT $=0.10$

MODEL, 23 , $29,25=1+3+5+7+9+11+12+13+14+15+16+17+18+19+20+21+22+26+28+30+32+34+$ $36+37+38+39+40+41+42+43+44+45+46+47+48+49$.

PRESS

ENO OF PARAMETERS (siat CONTROL CARO)

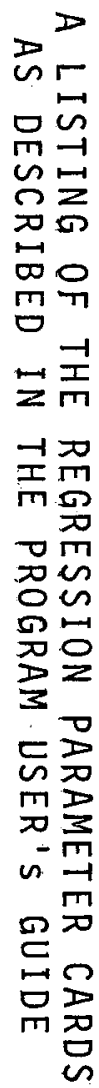




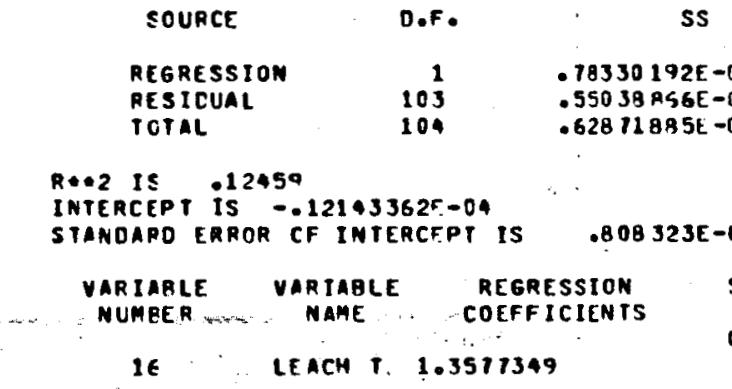

UNTOUE SEQUENCE NUMBER FOR THIS ANOUA = 10 B

STANOAROI ZED
REGRESSION. COEFFICIENTS .352969

MS F $.78330192 E-05$

$14.65^{n} 750$

$.64275 E-04$

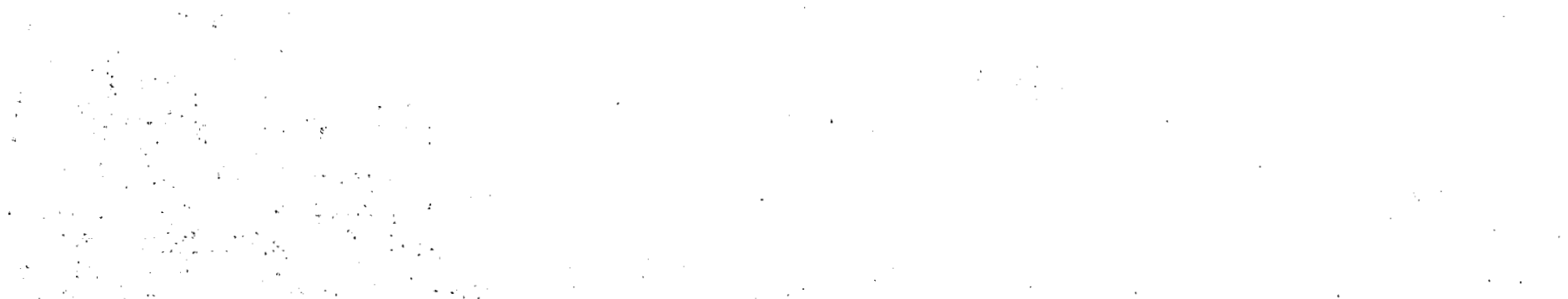

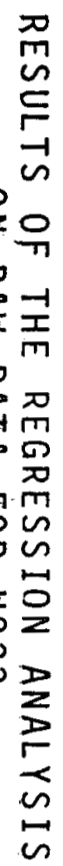

The Analys is on Raw Data Shows Only One Variable (Leach Time) to be Significant. 
SANCIA LABORATORIES $\$ STEPUISE REgRESSION FROGRAM $\$ COURTESY OF OEPT. OF STATISTICS - KanSAS STATE UNIVERSITY IILE,STEPUISE FOR NRC SHORT COURSE RANK TRANSFORHEO DATA VECT $1-105$

OATA, 25,24.2.
INPUT CHECK OF PARAMETERS

NUMBER OF VARIARLES REAO IN D 25

NO. OF TRAMSFORMED VARIABLES $=24$

DATA DISPOSITION IS 2

OUTPUT, CORR, STEPS

STEPUISE, SIGIN=0.05, SI GOUT $=0.10$

MOOEL $, 23,24,25=1+3+5+7+9+11+12+13+14+15+16+17+18+19+20+21+22+26+28+30+32+34+$

$36+37+38+39+40+41+42+43+44+45+46+47+4 B+49$.

PRESS

RAMK REGRESSION

END OF PARAMETERS
욘유

(STAT CONTROL CARD)

옴고

$\sum \frac{1}{m}$

的历

$\pi$

ᄁᄁ용

(stat control caros

(STAT CONTROL CARD)

wo

(STAT CONTROL CARO)

(STAT CONTROL CARD)

(STAT CONTROL CARC)

(STAT CONTROL CAROD 

TITLE,STEPWISE FCR NRC SHORT COURSE RANK TRANSFORMEO DATA VECT 1-105

SANDIA LABORATDRIES $\ C>$ STEPHISE REGRESSION $\<$ FROA KANSAS STATE UNIVERSITY

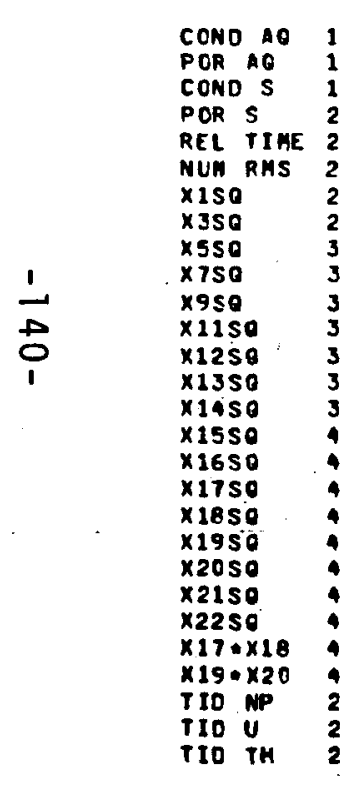

MAME
1.0000 1.0000
-.6708
.0206 .0206
.0047 $-.0342$ $-.0195$ $-.0167$ -.0948
-.0966 $\because 0096$ 0.1308 -.01348
-00356 $-.0040$ $-.0040$ .0207 $-.0203$ $-.0003$

.9688

$-.06635$

.0647

$-.0032$

.0351

$-.0010$

0

.5564

no.

CONO AO
CCRRELATION MATRIX

1.0000
-.0309
-.0111
.0387
.0036
.0642
.0907
.1131
.0989
.2465
-.0183
.0450
-.0713
-.0290
.0204
.0503
-.6450
.0688
-.0435
-.0135
.0249
.0085
.3209
-.0354
-.3396
-.3315
-.3605
.18

1.0000

$.7310: 1.0000$

$.0069 \quad .0054$

$.0036 \quad .0143$

.0122

.0425

$-.0259$

0.0260

$-.0260$

$-.0523$

$-.0063$

.0293

.00 .90

.0203

.0242

.0469

.6944

.0123

.0046

.0240

.9068

.1791

19

.0257

$-.0062$

.0004

$-.0131$

.0362

.0147

.0319

.0187

.0007

.0111

.9688

.0137

.0287

.0439

.8970

.1619

.1629

20
COND 5

POR 5

1.0000
.0471

1.0000

.0176

.0550

.0054

$-.0055$

$-.0308$

$-.0057$

.0150

.0063

$-.0152$

$-.0088$

$-.0043$

.9683

(.0522

.0021

$-.4800$

$-. .3992$

.0012
-.0194

$-.0194$

.0328

.0078
-00370

$-00370$

$-.0332$

-.0332
-.0305
-.00807

$-00807$

-.00453
.0652

.0652

.0405 .

.0204

.0617

.0574

.9688
-.0624

.00624
.0486

.00179

.0109
-0006

21

22

26

xiso

1.0000

.0835

.00252

.0597

.0290

.0071
-.0236

.0403

$-.0243$

.0083

.0677

.0587

.0400

.0090

.0197

NUM RMS

1.0000

-.0536
-.0294

(1)

.0732
.00307

.058

.0581
-.0193

-.0193
-.0007

$-00258$

$-.0063$

.00450

$-1097$

.0548
.0471

$-.0264$

.00264
.00098
.00556

$-.0556$

0.0087

$-0.0473$

28

350

1.0000
.0174
.1125
-.0127
-.0618
-.0543
.0175
.0358
-.0579
-.1045
.1482
-.0384
-.0026
.1015
.0260
-.0250
-.0186
-.1670
-.5277
-.3086

30

so $\begin{array}{rr}-.0188 & 1.0000 \\ .0069 & .0542\end{array}$

$.0620 \quad .0542$

$.0425 \quad .0067$

$.0138 \quad .0178$

.0244
.00220

$\begin{array}{r}.0123 \\ -.003\end{array}-.0378$

$.1224 \quad-2520$

$.00585 \quad-.0254$

$-.0196 \quad-.0079$

$-.0102 \quad-.0120$

$-.0614$

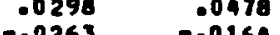

$-.02637-0.0164$

$.0212 \quad-.0651$

321

x9so 
SANOIÁ LABORATORIES $\ C$ STEPUISE REGRESSION $\longleftrightarrow\langle$ FROM KANSAS SIATE UNIVERSIT

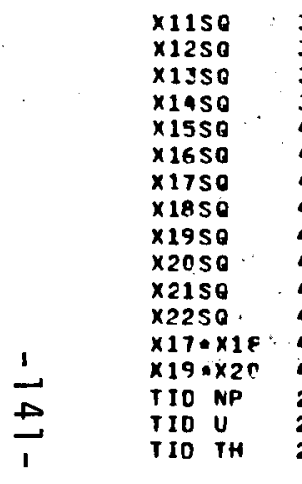

CCRRELATION MATRIX

\begin{tabular}{|c|c|}
\hline 36 & 1.0000 \\
\hline 37 & $\begin{array}{r}-.0042 \\
\end{array}$ \\
\hline 3 A & -.0095 \\
\hline 39 & .0004 \\
\hline 40 & .0275 \\
\hline 41 & .0035 \\
\hline 42 & -.0300 \\
\hline 43 & .0322 \\
\hline 44 & -.0774 \\
\hline 45 & -.0068 \\
\hline पe & -.7225 \\
\hline 47 & .0003 \\
\hline 48 & -.1314 \\
\hline 49 & -.0511 \\
\hline 23 & -.0158 \\
\hline 24 & -.0035 \\
\hline 25 & -.1071 \\
\hline No. & 36 \\
\hline
\end{tabular}

$\begin{array}{cc}1.0000 & \\ .0300 & 1.0000 \\ -.0169 & -00003 \\ .0177 & -.0351 \\ .0076 & .1268 \\ -.0099 & .0673 \\ -.0132 & -.0792 \\ .0295 & -.0075 \\ .0275 & -.0651 \\ -.0369 & -.0352 \\ -.0585 & -.0706 \\ .1307 & .0256 \\ .0246 & -.0250 \\ .0281 & .0458 \\ .0502 & .0575 \\ .0747 & .0580 \\ 37 & 38\end{array}$

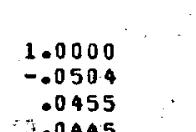

$.0455-0.000$

$\because .0445 \quad-0114$

.00428 .

$0.0089 \quad-.0132$

$-.0062 \quad-.0018$

.0157

$-.0061$

$.0183 \quad .0054$

$-.0152 \quad-.0054$

$.0311 \quad .0631$

$\begin{array}{rl}-.0213 & .0594 \\ -.1298 & .1237\end{array}$

NAME

$\times 1150$

$x 1250$

xisso

$\times 1450$

$\times 1550$

1.0009
.0003
.0839
.0185
-00212
-.0917
.0653
-.0103
.0065
.4043
.1968
.1279
11

1.0000

$-.6199 \quad 1.0000$

$.0647: \quad-0645$
$-.0138:$

.0505

$.0233 \quad .0415$

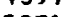

.
.
.
.3536

42

43

1.0000

.6833

.0149
.0346

.0346

.9223

0.2390

.1743

.2576

44

1.0000

.0047

.0438

.0922

.1385

1.0000 .0698
.0097

$\times 1650$

$\times 1750$

$\times 1850$

x19so

$\times 2050$

$\times 2150$

TITLE,STEPHISE FCR NRC SHORT COURSE RANK TRANSFORMEO DATA VECT 1-105

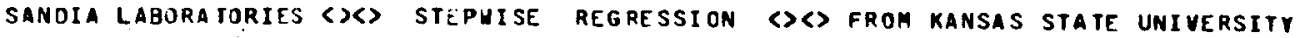

\begin{tabular}{|c|c|c|c|c|c|c|c|}
\hline \multirow{3}{*}{$\begin{array}{l}\times 2250 \\
\times 17 * \times 18 \\
\times 19 * \times 20\end{array}$} & \multirow[b]{2}{*}{47} & \multirow[b]{2}{*}{1.0000} & & \multicolumn{2}{|r|}{ C CRRELA TION } & \multirow[t]{2}{*}{ MATRIX } & \\
\hline & & & 1.0000 & & & & \\
\hline & 49 & .0687 & .0570 & 1.0000 & & & \\
\hline TIO NP & 23 & .0400 & .1716 & .1981 & 1.0000 & & \\
\hline $110 u$ & 24 & .0173 & .2387 & .1354 & .7671 & 1.0000 & \\
\hline TIO TH & 25 & -.0512 & .2801 & .2134 & .6766 & .7587 & 1.0000 \\
\hline & No. & 47 & 48 & $\bullet 9$ & 23 & 24 & 25 \\
\hline MAME & & so & $177 \times 18$ & $\times 19 \cdot \times 20$ & IID NP & T10 U & TIO TH \\
\hline
\end{tabular}


TITLE, SIEPUISE FCR NRC SHORT COURSE RANK TRANSFORMEO DATA VECT 1-205

SANDIA LABORATORIES (XS STEPUISE REGRESSION CSO FROR KANSAS STATE UNTUERSITY

ANALTSIS OF REGRESSIONLF FOR VARIABLE 2M---TIO U
CTAMLE II (TABLE 1 )

SOURCE

REGRESSION

RESICUAL
ICTAL
D.F.

1

$10 \frac{1}{104}$ ss

$31988 \cdot 195$

62933.915
94822.000

610.03704

MS

52.435463
SIGNIF ICANCE

.0000

R*A2 IS 033735

TIEPCEY IS $33.520 B 79$

STANDARO ERROR CF INTERCEPT IS 4.85538

\begin{tabular}{|c|c|c|c|}
\hline $\begin{array}{l}\text { VARIABLE } \\
\text { NUAEER }\end{array}$ & $\begin{array}{l}\text { VARIABLE } \\
\text { NAME }\end{array}$ & $\begin{array}{l}\text { REGRESSION } \\
\text { COEFFICIEN TS }\end{array}$ & $\begin{array}{l}\text { STANOAROIZED } \\
\text { REGRESSION } \\
\text { COEFFICIENTS }\end{array}$ \\
\hline
\end{tabular}

$-.580818$

$11 \mathrm{P}$

UNIQUE SEQUENCE MUMBER FOR THIS ANOVA = 11

RANK FIT GIVES A RAY OATA NORMALIZEO ROA2 $=.27533482$ E-OI

COEFFICIENT OF INTERPOLATION $=.27022919 E-01$

FRESS IS 64948. 
TIILE, SIEPHISE, FCR NRC SHORT COURSE' RANK TRANSFIRMED DATA VECT 1-105 SANDIA LABORATORIES ¿X STEPUISE REGRESSION ¿C FROM KANSAS STATE UNIVERSITY

ANALIS IS OF RFGRESSION FOR VARIABLE 24---TID U

(TABLE 11

$\begin{array}{lr}\text { SOURCE } & 0 . F \\ \text { REGRESS ION } & 2 \\ \text { RESIDUAL } & 102 \\ \text { TCIAL } & 104\end{array}$

$R+02$ IS .52432

IATERCEPT IS 105.09772
SIANOARD EAROR CF INTERCEPT IS

VARTABL

i

RF U $=.55368456$

COEFFICIENTS

$-.558446$

MS

$\mathbf{F}$

SIGNIFICANCE

$\begin{array}{lll}\$ 9717.436 & 24858.719 & 56.217802 \\ 45104.564 & 442.20161 & \end{array}$

.0000

UNIQUE SEOUENCE NUMBER FOR THIS ANOVA $=119$

RAMK FII GIVES A RAY OATA MORMALIZED R*.2 $=.26972345 E-01$

COEFFICIENT OF INTERPOLLTION $=.21997096$-OL

FRESS IS

97613. 
TITLE, STEPHISE FCR NRC SHORT COURSE RANK TRANSFORMEO DATA VECT 1-105

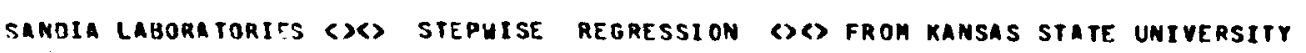

AOY TAHLE
ANALYSIS OF REGRESSION FOR VARIAGLE $24-\cdots-T$ IO $U$ (TAHLE 1 )

SOURCF.

REGRESSION RES ICUAL

D.F.

ss

101

104

65861.336
28960.654

99822.000
MS

21953.179

R०.2 IS .6545A

IATERCEPT IS E0.643474

STANOARD ERAOR CF INIERCEPT IS

5.10667

VARIABLE VARTABLT REgRESSION

NUMEE R

NAHE COEFFICIENTS

$5 \quad$ RF $10-0.51549520$

CONO AO 91111573

STANOARDTZED

REGRESSION

COEFICIENTS

- 519929

.419651
-.920183

SSO

25344.1328

16143.8996

UNIOUE SEOUENCE NUMBER FOR THIS ANOYA $=12$

RANK FIT GIVES A RAV DATA MORMALIZEO RA.2 = .50748162

COEFICIENT OF INIERPOLATION $=.1835 B 317 E-02$

PRESS IS 31213.

PRESS IS 31213.
SIGNIFICANCE

76.563562

.0000

$\begin{array}{lr}\text { T-TEST } & \text { REA2 } \\ \text { VALUES } & \text { DELETES } \\ -9.9015 & .4273 \\ 7.5034 & .5293 \\ -7.6384 & .5181\end{array}$

ALPMA

.0000

.0000
.0000 
SUMMARY OF STEPWISE REGRESSION ON RAW DATA

\begin{tabular}{|c|c|c|c|c|c|c|}
\hline Vectors & NP 237 & $\mathrm{R}^{2}$ & $\underline{\mathrm{U} 233}$ & $\mathrm{R}^{2}$ & TH229 & $\mathrm{R}^{2}$ \\
\hline $1-35$ & Cond $S$ & .15 & Leach $T$ & .18 & $\begin{array}{l}\text { No. Rms. } \\
\text { Rel Time } \\
\text { Por S }\end{array}$ & .33 \\
\hline $36-70$ & $\begin{array}{l}(\text { Por } A)^{2} \\
\text { Por } A \\
\text { Leach }\end{array}$ & .66 & $\begin{array}{l}\text { Leach } \mathrm{T} \\
\text { S. Lim } \mathrm{Np} \\
(\text { Leach } \mathrm{T})^{2}\end{array}$ & .55 & $\begin{array}{l}\text { Leach } T \\
\text { So Lim Np } \\
\text { (Leach T) }\end{array}$ & .56 \\
\hline $71-105$ & Rel Time & .12 & S. Lim Np & .57 & $\begin{array}{l}\left(S . \operatorname{Lim}_{T h}\right)^{2} \\
\text { S. } \operatorname{Lim}_{N p} \\
\text { S. Lim Th } \\
\text { RF A Np }\end{array}$ & .97 \\
\hline $1-105$ & $\begin{array}{l}\text { Rel Time } \\
\text { Cond } S \\
\text { (Cond } S)^{2} \\
\text { Leach T }\end{array}$ & .27 & Leach T & .12 & $\begin{array}{l}\text { Por S } \\
\text { Rel Time } \\
\text { No. Rms }\end{array}$ & .19 \\
\hline
\end{tabular}

Note the inconsistency of variable selection

from one set of runs to the next for this

analysis on raw data 


\section{SUMMARY OF STEPWISE REGRESSION ON RANKS}

\begin{tabular}{|c|c|c|c|c|c|c|}
\hline Vectors & NP237 & $\mathrm{R}^{2}$ & $\underline{U} 233$ & $R^{2}$ & TH229 & $\mathrm{R}^{2}$ \\
\hline $1-35$ & $\begin{array}{l}\text { Cond A } \\
\text { Rel Time } \\
\text { RF A Np } \\
\text { Leach T }\end{array}$ & $.57(.74)$ & $\begin{array}{l}\text { Rel Time } \\
\text { Cond A } \\
\text { RF A U }\end{array}$ & $.99+(.78)$ & $\begin{array}{l}\text { Cond A } \\
\text { RF A TH } \\
\text { Rel Time }\end{array}$ & $.94(.83)$ \\
\hline $36-70$ & $\begin{array}{l}\text { Leach T } \\
\text { Rel Time } \\
\text { RF A Np } \\
\text { Cond A } \\
\text { Cond S }\end{array}$ & $.06(.86)$ & $\begin{array}{l}\text { RF A U } \\
\text { Rel Time } \\
\text { Cond A } \\
\text { Por S } \\
\text { Leach T }\end{array}$ & $.64(.82)$ & $\begin{array}{l}\text { Cond A } \\
\text { Rel Time } \\
\text { RF A TH } \\
(\text { RF A TH })^{2}\end{array}$ & $.89(.79)$ \\
\hline $71-105$ & $\begin{array}{l}\text { RF A NP } \\
\text { Rel Time } \\
\text { Leach T } \\
\text { Cond A } \\
\text { Cond S }\end{array}$ & $.41(.81)$ & $\begin{array}{l}\text { RF A U } \\
\text { Rel Time } \\
\text { Cond A } \\
\text { Cond S } \\
(\operatorname{RF} A U)^{2}\end{array}$ & $.47(.74)$ & $\begin{array}{l}\text { Cond A } \\
\text { RF A TH } \\
\text { Rel Time } \\
\text { RF A Np } \\
\text { (RF A TH) } 2\end{array}$ & $.41(.68)$ \\
\hline $1-105$ & $\begin{array}{l}\text { RF A Np } \\
\text { Rel Time } \\
\text { Leach T } \\
\text { Cond A }\end{array}$ & $.48(.73)$ & $\begin{array}{l}\text { RF A U } \\
\text { Rel Time } \\
\text { Cond A }\end{array}$ & $.51(.69)$ & $\begin{array}{l}\text { Cond A } \\
\text { RF A TH } \\
\text { Rel Time } \\
\text { RF A U } \\
\text { Cond S }\end{array}$ & $.59(.75)$ \\
\hline
\end{tabular}

These are the variables selected as important by the stepwise regression analysis on ranks. This selection agrees well with the variables identified as important by the PRCC on pages 114 to 118. Note that the notation RF A used here means retardation factor (RF) in the aquifer and is calculated using the KD values listed with the PRCC. Likewise K UP AQ and Cond $A$ both refer to conductivity in the upper aquifer. 
